\title{
Structural characterization of $\alpha$-synuclein aggregates seeded by patient material
}

\author{
Dissertation \\ to acquire the doctoral degree in mathematics and natural sciences \\ 'Doctor rerum naturalium' \\ at the Georg-August-Universität Göttingen \\ in the doctoral program of Biology \\ at the Georg-August University School of Science (GAUSS) \\ participating in the International PhD Program in \\ Molecular Biosciences and Biomedicine \\ of the Universidad Nacional de Rosario in Argentina \\ and the Georg-August-Universität Göttingen
}

Submitted by

Timo Strohäker

from Herrenberg

Göttingen, 2018 


\section{Thesis Committee}

Prof. Dr. Markus Zweckstetter

Structure Determination of Proteins Using NMR, Max-Planck-Institute for Biophysical Chemistry, Göttingen

Translational Structural Biology in Dementia, German Center for Neurodegenerative Diseases, Göttingen

Prof. Dr. Tiago Outeiro

Department of Experimental Neurodegeneration, University Medical Center (UMG) Göttingen

Dr. Claudio Fernández

Max-Planck Laboratory for Structural Biology, Chemistry and Molecular Biophysics of Rosario (LMPbioR), CONICET IIDEFAR, Universidad Nacional de Rosario (UNR), Rosario (Argentina)

\section{Members of the Examination Board}

Reviewer: $\quad$ Prof. Dr. Markus Zweckstetter

Second Reviewer: $\quad$ Dr. Claudio Fernández

\section{Further members of the Examination Board:}

Prof. Dr. Holger Stark

Department Stark, Structural Dynamics, Max-Planck-Institute for Biophysical Chemistry, Göttingen

Dr. Mauricio Menacho Márquez

Max-Planck Laboratory for Structural Biology, Chemistry and Molecular Biophysics of Rosario (LMPbioR), CONICET IIDEFAR, Universidad Nacional de Rosario (UNR), Rosario (Argentina)

Dr. Sebastian Kügler

Department of Neurology, Viral Vectors Lab, University Medical Center (UMG) Göttingen 


\section{Affidavit}

I hereby declare that the doctoral thesis entitled "Structural characterization of $\alpha$ synuclein aggregates seeded by patient material" has been written independently, with no other sources and aids than quoted.

Timo Strohäker

Göttingen, November 2018 


\section{Acknowledgements}

I would like to thank Prof. Markus Zweckstetter for giving me the chance to work on this exciting and challenging research project and for the constant help and guidance that he has provided me with at all stages of my doctoral studies as well as his openness and support to let me join the binational doctoral program. It was more than a pleasure to work in such an international and diverse research group having access to outstanding equipment and addressing highly relevant scientific questions.

I would also like to thank Dr. Claudio Fernández for his supervision and scientific input as well as the extraordinary research experience that I enjoyed during my stay at his group at the Max Planck Laboratory of Rosario (LMPbioR) in Argentina. My time in this beautiful country was something truly special. I would also like to thank him for his extraordinary commitment to solving all obstacles related to the exchange program.

Thank goes as well to Prof. Tiago Outeiro and Prof. Holger Stark for their scientific advice and support.

In addition, I would like to show my gratitude to Prof. Seung-Jae Lee and Dr. Byung Chul Jung of the Department of Biomedical Sciences of the Seoul National University College of Medicine, Prof. Marina Bennati and Dr. Shu-Hao Liou of the EPR spectroscopy group of the MPIBPC in Göttingen, Dr. Stefan Becker, Karin Giller and Kerstin Overkamp of the Department of NMR-based Structural Biology of the MPIBPC in Göttingen as well as Prof. Glenda M. Halliday and Prof. Woojin Kim of the Brain and Mind Centre of the University of Sidney for their scientific contributions.

A very big thank goes to all these brilliant, interesting and highly skilled people that I have met during all those years and that I have had the luck to share some time with. I feel deeply thankful to all of you. Your help and instructions, the constant support, the fruitful scientific discussions, your encouragements and recommendations altogether have enabled me to achieve my goals and shaped me during the time as a doctoral student and as a person in general. Thank you for making my life inside and outside the lab so worthwhile. I would like to specially mention Hessam, Filippo, Andrés B., Taekyung, Javier, Yunior, Hari, Piotr, Garima, Martin, Aldo, Frederik, Luis, Adriana, Juan Carlos, Dirk, Kris, Mariusz and Lukasz, Sol, Conny, Alain, Antonia, Susmitha, Pijush, Marija, Maria, Tina, Mauge, Caterina, Gonzalo, Marco, Andrés T. and Franco. 
I would also like to thank Prof. Griesinger and Dr. Claudio Fernández for establishing the German-Argentine doctoral program. I highly value their persistence and the enormous amount of work that they have put behind this international cooperation. I will always be thankful that I could live and work in Argentina and that I had the chance to get to know the country and its people at first hand. The ties and bridges that are shaped by such programs will endure a lifetime and eventually will bring the two countries closer together and create synergies.

Thanks to Prof. Griesinger and the whole staff of the NMR2 research group that I was not only sharing equipment with, but that I could always address with whatever question or matter and received a helping hand.

Thanks also to Petra and Dirk of NMR2, Daniel and Ulrike of the DZNE as well as Laura, Marianela and Mauricio of the LMPbioR for all the valuable administrative support.

Thanks as well to the additional members of my examination board Dr. Mauricio Menacho Márquez and Dr. Sebastian Kügler.

Querida Flor, without the constant and unconditional support that I always enjoyed from you, all of this now almost appears inconceivable. Thank you for helping me through setbacks and disappointments that inevitably occurred over time on this journey. Your endless optimism and energy are a true source of inspiration and my gratefulness to you is best described by these two words: te amo!

In the same way my gratitude and love go to my family and friends. Mama and Papa, Oma and Opa as well as my siblings Bernd, Ralf and Christin. You were always there for me and backed me from my earliest beginnings on and I know that I can always count on you. Thank you for always reminding me on the real important things in life. Danke! 


\section{Abstract}

Neurodegenerative diseases share a common underlying pathologic hallmark, the appearance of insoluble protein aggregates in diverse tissues of the nervous system. For many neurodegenerative diseases a common temporal and spatial spreading of the pathology is proposed in analogy to prion disease and discussed under the term "prion-like". For many diseases the major component of the insoluble protein aggregates is known and aggregation into higher molecular weight amyloid fibrils with intermolecular $\beta$-sheet rich cores can be studied in vitro. The aggregation process involves the templated misfolding and aggregation of native monomeric proteins, involving severe conformational changes.

An important family of neurodegenerative diseases is caused by the misfolding and aggregation of the protein $\alpha$-synuclein, the so-called synucleinopathies. $\alpha$ synuclein, which in vivo forms disease-specifically the main component of intracellular inclusions such as Lewy bodies in neurons and cytoplasmatic inclusions in glial cells, undergoes in vitro dramatic conformational changes from a monomeric intrinsically disordered state over transient oligomeric $\beta$-sheet rich species into highly ordered $\alpha$ synuclein fibrils.

$\alpha$-synuclein pathology in patients is diverse and there are clinically distinct disease entities with defined pathologic phenotypes among those Parkinson's disease (PD), dementia with Lewy bodies (DLB) and multiple system atrophy (MSA) are the best characterized. Similar to prion diseases, key differences within the broad clinical representation of synucleinopathies are thought to be structurally encoded by distinct protein aggregate conformations, referred to as $\alpha$-synuclein polymorphs.

The aim of the study was to amplify $\alpha$-synuclein aggregates from brain extracts of patients thoroughly diagnosed on the basis of the molecular pathology as well as the clinical symptoms as PD, DLB and MSA, using the established protocol of protein misfolding cyclic amplification (PMCA). A combination of hydrogen-deuterium (HD) exchange coupled to nuclear magnetic resonance (NMR) spectroscopy, electron paramagnetic resonance (EPR) and the specific binding of fluorescent probes to amyloid fibrils was chosen to obtain single-residue resolution of the conformational properties of brain-extract seeded $\alpha$-synuclein fibrils. The same approach was also applied to two well-characterized in vitro $\alpha$-synuclein polymorphs, their aggregation was performed in the absence of brain extract seeds following published aggregation procedures and they acted as internal references for benchmarking the methodological approach. On the other hand, the availability of a high-resolution cryo-electron 
microscopy model of the fibrillar core for one of the in vitro $\alpha$-synuclein polymorphs obtained under high salt conditions, allowed direct correlation of the residue-specific conformational restraints to a structural model, both for in vitro polymorphs of $\alpha$ synuclein as well as brain-extract amplified $\alpha$-synuclein fibrils of PD, MSA and DLB.

Distinct highly ordered conformational features of in vitro $\alpha$-synuclein fibrils were successfully reproduced, detecting solvent-protected residues with high precision and in agreement with published data. In contrast, $\alpha$-synuclein fibrils amplified from brain extracts were more flexible and differed structurally from in vitro fibrils. Hydrogendeuterium exchange coupled to NMR spectroscopy identified a common solventprotected core shared among all patient brain derived $\alpha$-synuclein fibrils for the synucleinopathies PD, MSA and DLB. The solvent-protected fibrillar core was formed by the most hydrophobic residues of $\alpha$-synuclein. Outside the common core structure, $\alpha$-synuclein fibrils derived from brain extracts differed disease-specifically in the conformation. Residue-specific conformational differences in core-flanking residues of $\alpha$-synuclein as well as in defined $\mathrm{N}$-terminal regions were observed.

This study establishes a strong correlation between $\alpha$-synuclein aggregate structure and the disease phenotype for the synucleinopathies Parkinson's disease, Dementia with Lewy bodies and multiple system atrophy and the data provide further insight in "prion-like" features of neurodegenerative diseases in general and synucleinopathies in particular. The work presented here is a step forward towards the understanding of $\alpha$-synuclein pathology and hopefully contributes to improved disease diagnosis and treatment of synucleinopathies. 


\section{Publications}

Following publications appeared in peer-reviewed journals as a doctoral student including personal contributions as stated in the respective publications.

Moree, B. et al.

Small Molecules Detected by Second-Harmonic Generation Modulate the Conformation of Monomeric alpha-Synuclein and Reduce Its Aggregation in Cells.

J Biol Chem 290, 27582-27593, (2015)

Gentile, l. et al.

Interaction of $\mathrm{Cu}(\mathrm{i})$ with the Met-X3-Met motif of alpha-synuclein: binding ligands, affinity and structural features.

Metallomics 10, 1383-1389, (2018). 


\section{Table of Contents}

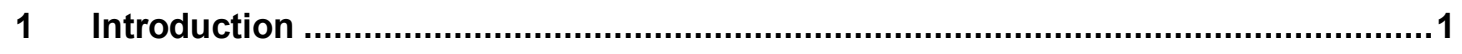

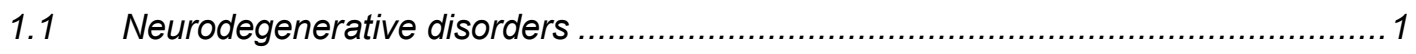

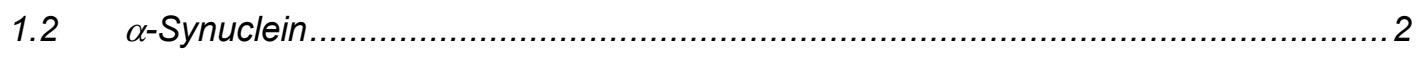

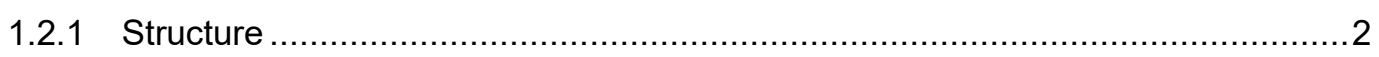

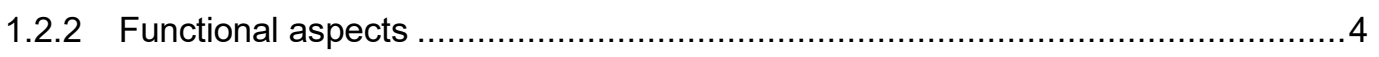

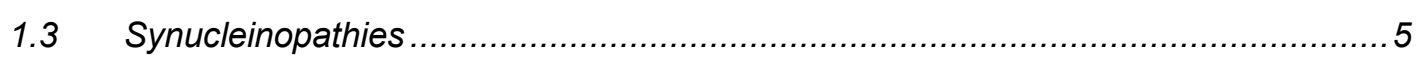

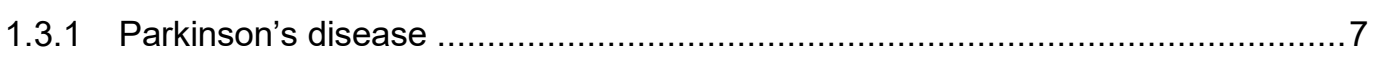

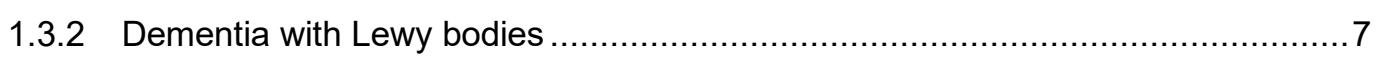





1.4 Prion hypothesis and prion-like behavior of aSyn.......................................... 8

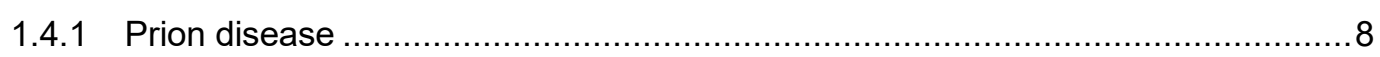

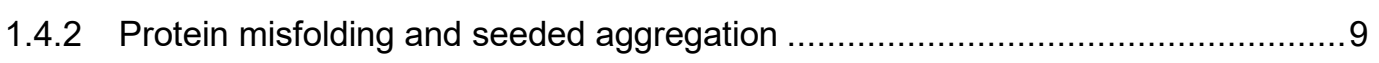



1.4.4 Prion strain-like behavior in synucleinopathies and structural polymorphism .....12

1.4.5 Fluorescent dyes as molecular probes for disease-specific conformations ........ 13

1.5 High resolution structures of in vitro aSyn fibrils........................................... 13

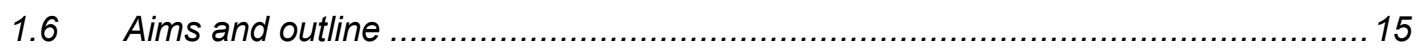



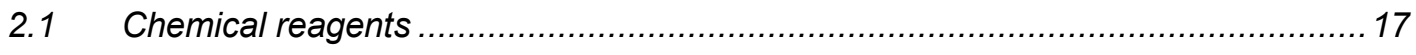



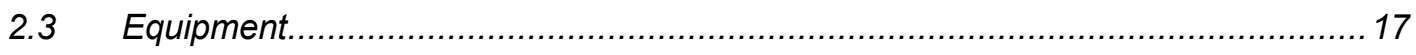

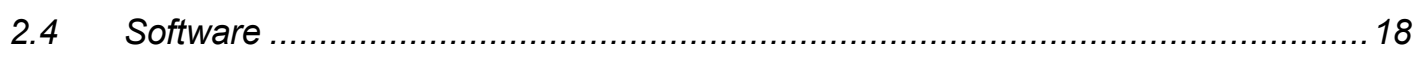

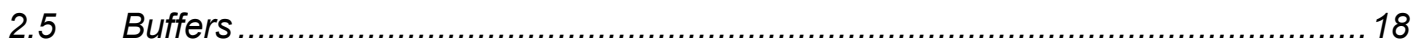

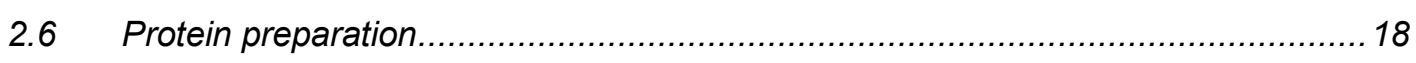

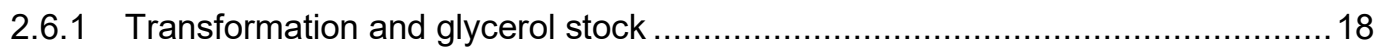

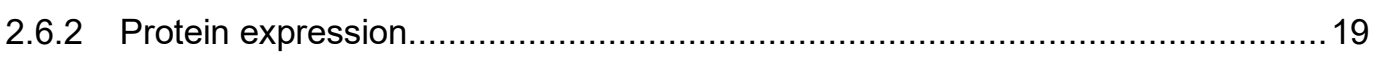

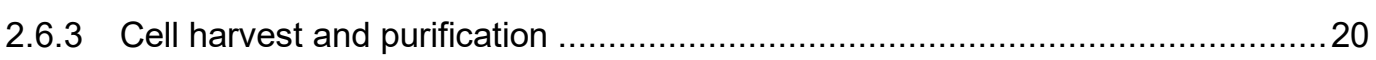



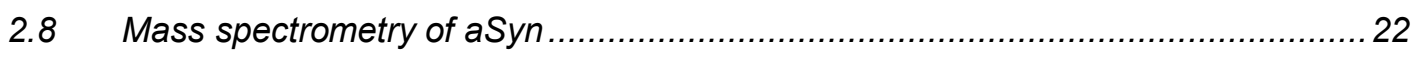

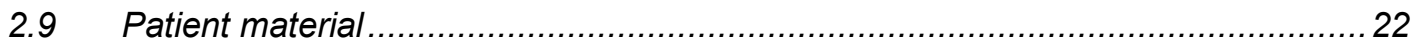

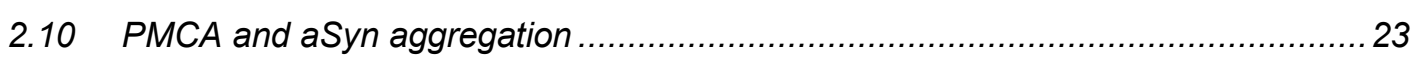


2.10.1 Homogenization of patient's material and PMCA ............................................

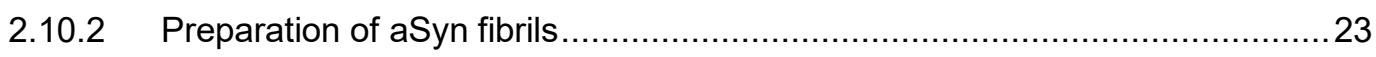

2.11 Negative stain transmission electron microscopy .........................................25

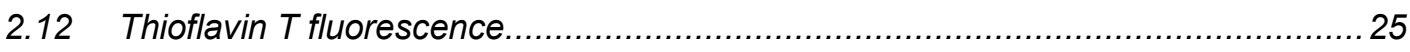

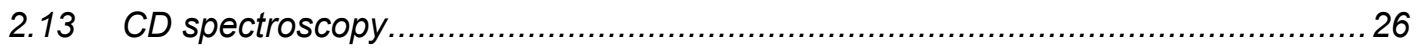

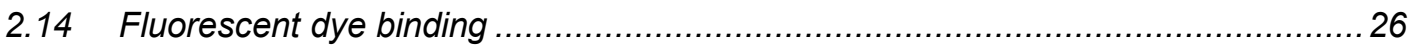



2.15.1 Heteronuclear single quantum coherence (HSQC) ................................28

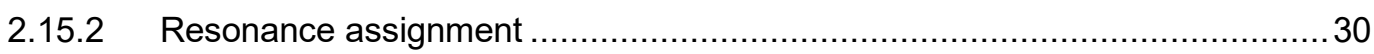

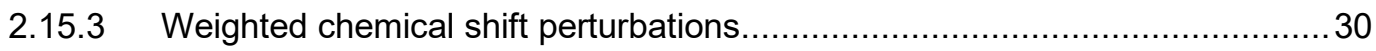

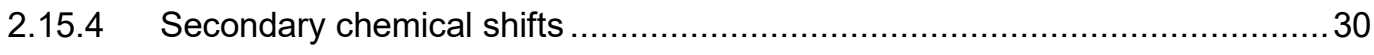

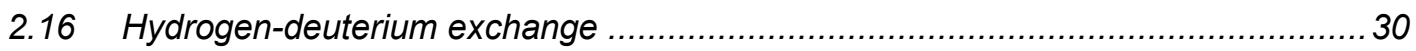

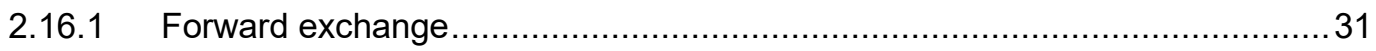

2.16.2 Back-exchange and NMR real-time acquisition ......................................... 31

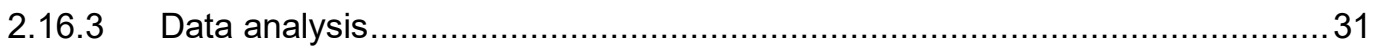

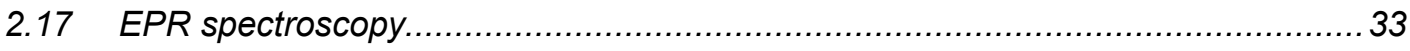



3.1 Biophysical characterization of monomeric aSyn ............................................. 36

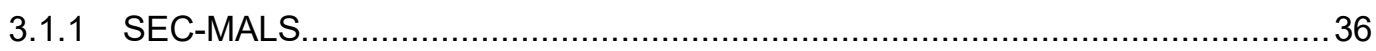

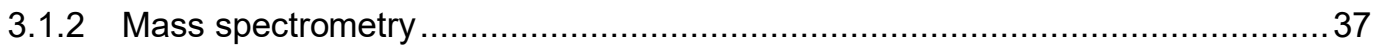

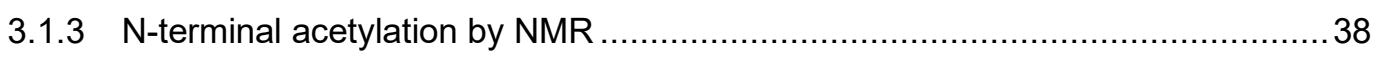



3.2.1 Amplification of aSyn aggregates from brain extracts ...................................40

3.2.2 Aggregation of high and low salt in vitro polymorphs...................................4 41

3.3 Characterization of aSyn aggregates by EM ............................................. 42

3.3.1 EM of aSyn aggregates amplified from patient brain material by PMCA ............42

3.3.2 EM pictures of high and low salt in vitro polymorphs ......................................43

3.4 Secondary structure analysis of aSyn fibrils by CD spectroscopy ........................ 43

3.5 Detection of conformational differences by fluorescent dye binding ...................... 44

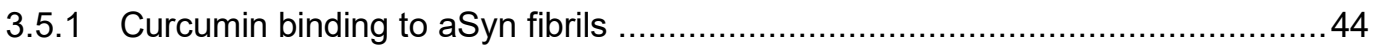

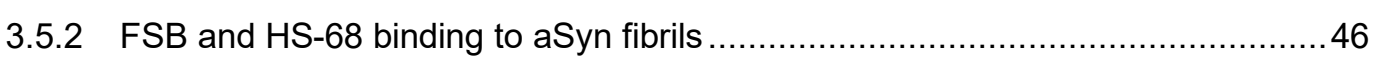

3.6 Hydrogen-deuterium exchange coupled to NMR ......................................... 47

3.6.1 Assignment of aSyn resonances in dissociation buffer .................................. 48

3.6.2 Hydrogen-deuterium back-exchange in dissociation buffer .............................50

3.7 HD exchange profile of the in vitro high and low salt polymorphs ........................54 
3.7.1 HD exchange profile of the high salt in vitro polymorph .................................54

3.7.2 Robustness of the high salt in vitro polymorph HD exchange profile .................57

3.7.3 HD exchange profile of the in vitro low salt polymorph ....................................58

3.7.4 Comparison of high- and low salt in vitro polymorphs...................................58

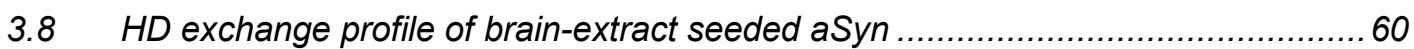



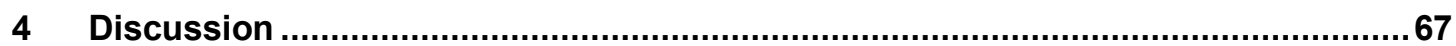

4.1 Evaluation HD exchange coupled to NMR spectroscopy .................................67

4.1.1 Accessibility of structural polymorphism by HD exchange ...............................67

4.1.2 Protonation levels in aSyn fibrils seeded by brain extracts ..............................68

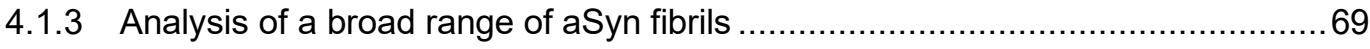

4.2 Amyloid polymorphism in other neurodegenerative diseases .............................. 70

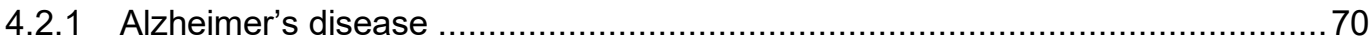



4.2.3 Disease-associated amyloid polymorphism in tauopathies and synucleinopathies 73

4.2.4 Structural characterization of $A \beta$ aggregates from $A D$ patient brain material .....74

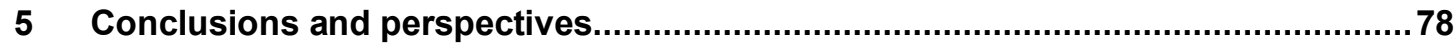

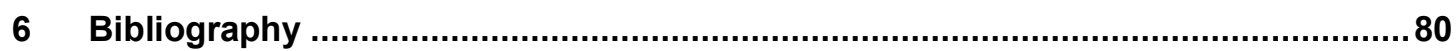

$7 \quad$ Appendix

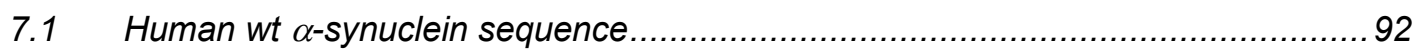

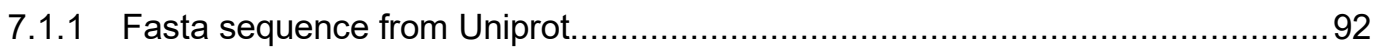

7.1.2 Physical and chemical parameters of $\alpha$-synuclein ........................................92

7.2 Resonance assignment of aSyn in dissociation buffer ....................................... 92

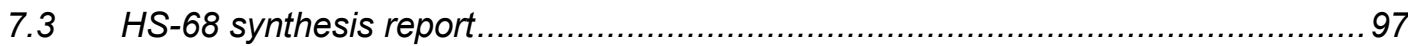

7.4 Mass spectrometry of non-acetylated $\alpha$-synuclein .......................................... 98

7.5 Pulse sequence used for real-time HD back-exchange ....................................... 99



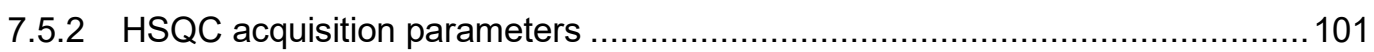

7.6 Residue-specific protonation levels of aSyn fibrils......................................... 102

7.6.1 aSyn fibrils seeded by brain extract of patient brain .................................. 102




7.7 Dipolar modulation of aSyn in vitro polymorphs analyzed by DEERNet neural network

7.8 Biophysical characterization of $\alpha$-synuclein membrane interactions and the close homologue $\beta$-synuclein 109

7.8.1 Protein-membrane interactions by NMR spectroscopy .... 109

7.8.2 Protein-membrane interactions by CD spectroscopy 110

\section{Index of Tables}

Table 1. Overview of common neurodegenerative disorders and the disease defining

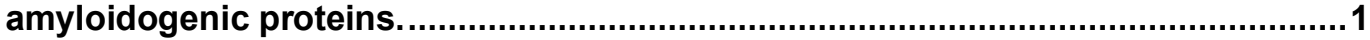

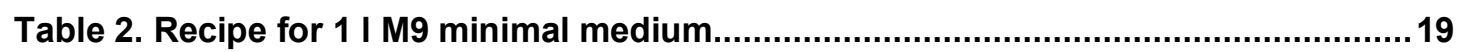

Table 3. Demographics of donor brains from synucleinopathy patients........................22

Table 4. 3D experiments recorded for resonance assignment of aSyn in dissociation

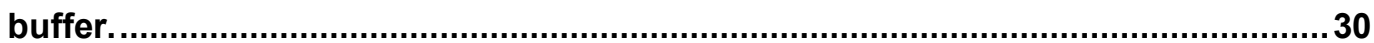

Table 5. Resonance assignment of aSyn in dissociation buffer. ...................................96



Table 7. Back-fitted initial protonation levels of brain-extract seeded aSyn fibrils.....104

Table 8. Back-fitted initial protonation levels of aSyn in vitro polymorph fibrils. ........107 


\section{Table of Figures}

Figure 1-1. Antibody staining of hallmark protein deposits. ........................................



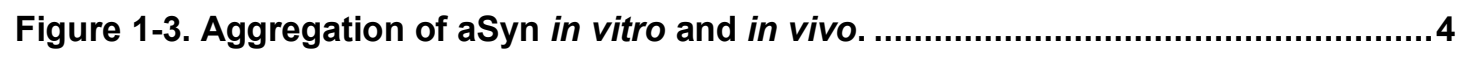

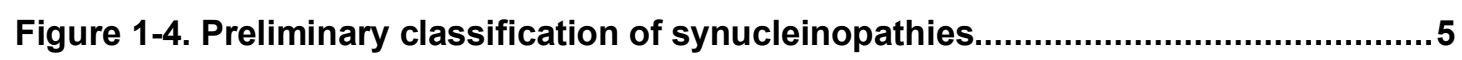

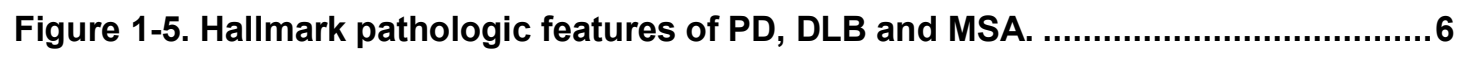

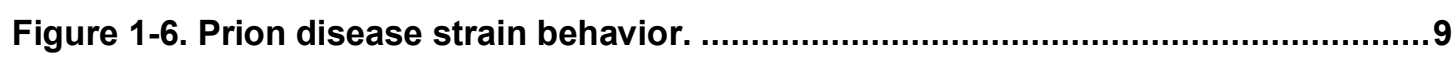

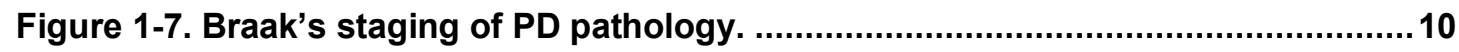

Figure 1-8. Strain behavior in neurodegenerative diseases. .......................................13

Figure 1-9. High resolution structures of in vitro aSyn fibrils.....................................15

Figure 2-1. Purification of $\mathrm{N}$-terminally acetylated $\alpha$-synuclein..................................21

Figure 2-2. Basic principle of cyclic amplification of aSyn fibrils using PMCA..............23

Figure 2-3. Aggregation scheme for PMCA end products..........................................25

Figure 2-4. Overview of CD spectra of pure secondary structures................................26

Figure 2-5. Chemical structural formula of fluorescent dyes.......................................27

Figure 2-6. Simplified scheme of 2D HSQC NMR spectroscopy. ..................................28

Figure 2-7. Schematic description of HD exchange coupled to NMR spectroscopy......32

Figure 2-8. MTSL-labeled aSyn A90C/T54C incorporated into fibril structure at 1:30....34

Figure 3-1. SEC-MALS of aSyn in comparison to non-acetylated $\alpha$-synuclein................37

Figure 3-2 Validation of $\mathrm{N}$-terminal acetylation of aSyn by mass spectrometry. ............38



Figure 3-4. Amplification of aSyn from brain extracts. ............................................40

Figure 3-5. Electron micrographs of aSyn fibrils amplified from brain extracts. ...........42

Figure 3-6. Electron micrographs of aSyn in vitro polymorphs..................................43

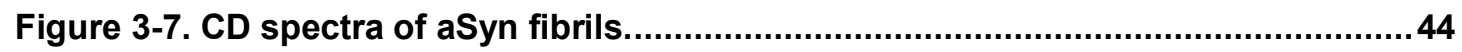

Figure 3-8. Curcumin binding to aSyn fibrils..........................................................45

Figure 3-9. Absolute fluorescence of curcumin binding to aSyn fibrils. .......................46

Figure 3-10. FSB and HS-68 binding to aSyn fibrils................................................... 47

Figure 3-11. Secondary chemical shift differences..................................................48

Figure 3-12. HSQC spectrum of aSyn in dissociation buffer.......................................49

Figure 3-13. HD back-exchange monitored by HSQCs in real-time................................51

Figure 3-14. Back-exchange curves of hsAsyn polymorph........................................52

Figure 3-15. Data transformation from intensities to absolute protonation levels..........53

Figure 3-16. Residue-specific protonation levels in high salt polymorph (hsAsyn) fibrils

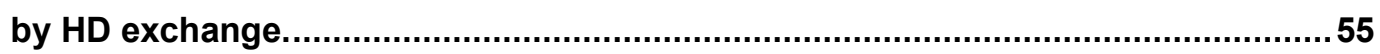

Figure 3-17. HD exchange profile mapped on high-resolution cryoEM structure..........56

Figure 3-18. HD exchange profiles for in vitro high salt polymorph control experiments. 
Figure 3-19. Residue-specific protonation levels in low salt polymorph (IsAsyn) fibrils



Figure 3-20. Comparison of HD exchange profile of high and low salt in vitro polymorphs

Figure 3-21. Residue-specific protonation levels of brain-extract seeded aSyn fibrils.. 61 Figure 3-22. Conformational properties of aSyn fibrils amplified from PD, MSA and DLB

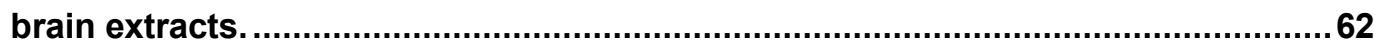

Figure 3-23. Extended data for brain-extract seeded fibrils. ......................................63

Figure 3-24. Continuous wave EPR spectra of aSyn fibrils.........................................64

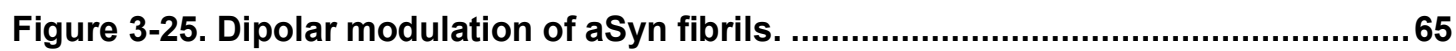

Figure 3-26. Dipolar modulation of aSyn fibrils, extended information. .........................66

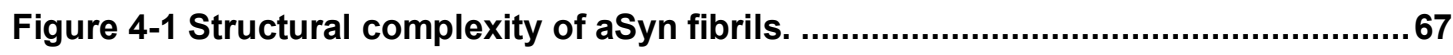

Figure 4-2. Different packing arrangements of the same protofilament kernel lead to



Figure 4-3. Cross-sections of the PHF and SF cryo-EM structures. ............................71

Figure 4-4. Polymorphic structure of tau inclusions in $\mathrm{AD}$ and $\mathrm{PiD}$ in comparison.......72

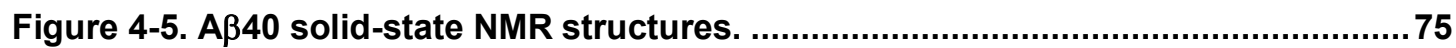

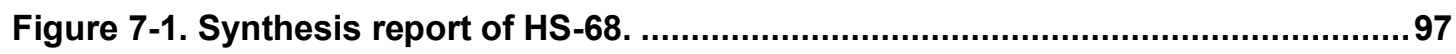

Figure 7-2. Mass spectrometry of non-acetylated $\alpha$-synuclein ...................................98

Figure 7-3. Dipolar modulation of aSyn fibrils using DEERNet...................................108

Figure 7-4. SUV titration of $\alpha$ - and $\beta$-synuclein by NMR spectroscopy........................110

Figure 7-5. SUV titration of $\alpha$ - and $\beta$-synuclein by CD spectroscopy...........................111 


\section{Abbreviations}

\begin{tabular}{|c|c|}
\hline aSyn & $\mathrm{N}$-terminally acetylated $\alpha$-synuclein \\
\hline CJD & Creutzfeldt-Jakob disease \\
\hline DLB & Dementia with Lewy bodies \\
\hline EM & Electron microscopy \\
\hline EPR & Electron paramagnetic resonance \\
\hline \multirow[t]{2}{*}{ FSB } & (E,E)-1-fluoro-2,5-bis(3-hydroxycarbonyl-4-hydroxy) \\
\hline & styrylbenzene \\
\hline $\mathrm{GCl}$ & Glial cytoplasmatic inclusions \\
\hline HD & Hydrogen-deuterium \\
\hline \multirow[t]{2}{*}{ hsAsyn } & High salt aSyn polymorph (50 mM Tris- $\mathrm{HCl}, \mathrm{pH} 7.5,150 \mathrm{mM}$ \\
\hline & $\left.\mathrm{KCl}, 0.02 \% \mathrm{NaN}_{3}\right)$ \\
\hline HSQC & Heteronuclear single quantum coherence/correlation \\
\hline IDP & Intrinsically disordered protein \\
\hline IsAsyn & Low salt aSyn polymorph (5 mM Tris- $\left.\mathrm{HCl}, \mathrm{pH} 7.5,0.02 \% \mathrm{NaN}_{3}\right)$ \\
\hline MSA & Multiple system atrophy \\
\hline \multirow[t]{2}{*}{ MTSL } & S-(1-oxyl-2,2,5,5-tetramethyl-2,5-dihydro-1H-pyrrol-3-yl) \\
\hline & methyl methanesulfonothioate \\
\hline NAC & Non-A $\beta$ component \\
\hline NMR & Nuclear magnetic resonance \\
\hline NTFs & Neurofibrillary tangles of tau protein \\
\hline NUS & Non-uniform sampling \\
\hline PAGE & Polyacrylamide gel electrophoresis \\
\hline PD & Parkinson's disease \\
\hline PFF & Pre-formed fibrils \\
\hline PHF & Paired-helical filaments of tau in AD \\
\hline PiD & Pick's disease, also called frontotemporal dementia \\
\hline PMCA & Protein misfolding cyclic amplification \\
\hline PrP & Prion protein \\
\hline QELS & Quasielastic light scattering \\
\hline SDS & Sodium dodecyl sulfate \\
\hline SEC & Size-exclusion chromatography \\
\hline SF & Straight filaments of tau in AD \\
\hline SN & Supernatant \\
\hline UC & Ultracentrifugation \\
\hline
\end{tabular}





\section{Introduction}

\subsection{Neurodegenerative disorders}

Age-related ${ }^{1}$ neurodegenerative disorders form a class of heterogeneous diseases, differing in pathology and clinical symptoms. The common hallmark and class-defining pathology is the intra- and extracellular aggregation of particular proteins in the human brain (Table 1). They are therefore also named proteinopathies. Figure 1-1 shows three examples of aggregated protein deposits. These protein deposits are also often referred to as amyloids in analogy to the polysaccharide amylose ${ }^{2}$. Amyloids as well as starch reacts positively with iodine, that allowed histological staining early on.

\section{Neurodegenerative disease}

Parkinson's disease ${ }^{3}$

Alzheimer's disease ${ }^{5}$

Amyotrophic lateral sclerosis ${ }^{8}$

Huntington's disease ${ }^{11}$

Creutzfeldt-Jakob disease ${ }^{13}$

\section{Amyloidogenic proteins}

$\alpha$-synuclein ${ }^{4}$

$\mathrm{A} \beta^{6}, \mathrm{tau}^{7}$

TDP $-43^{9}$, SOD $1^{10}$

Huntingtin protein $^{12}$

Prion protein ${ }^{14}$

Table 1. Overview of common neurodegenerative disorders and the disease defining amyloidogenic proteins.

a

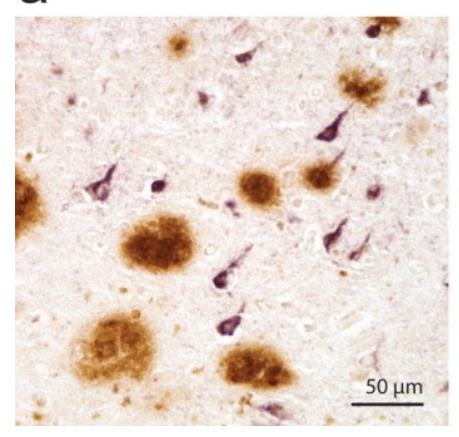

b

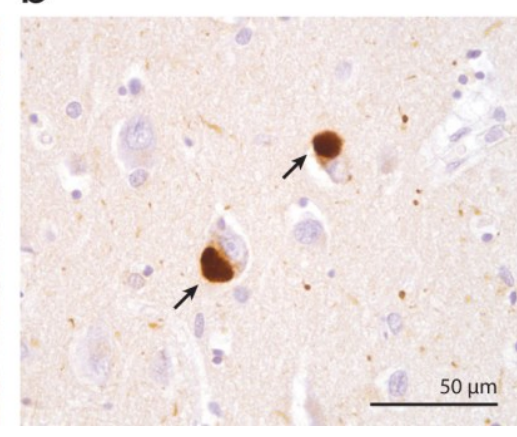

C



Figure 1-1. Antibody staining of hallmark protein deposits.

(a) In brown extracellular $A \beta$ plaques and in purple intracellular neurofibrillary tau tangles of AD. (b) Lewy bodies (arrow) common in PD and DLB. (c) TDP-43 (arrow) deposits of ALS, taken from Walker $\mathrm{LC}^{15}$.

Neurodegeneration is a complex multifactorial interplay ${ }^{16}$ that differs from one disease to the other, but it always manifests in the loss of neuronal and non-neuronal glia cells due to cell death in the brain over time. In an aging society with growing life expectancy, age-related neurodegenerative disorders have become a heavy burden for the health care systems and they are a major public health priority for policy makers worldwide ${ }^{17}$. 


\section{$1.2 \alpha-S y n u c l e i n$}

The human protein $\alpha$-synuclein (aSyn) is 140 amino acids long and has a molecular weight of $14.46 \mathrm{kDa}$. The human gene SNCA is located on the long arm of chromosome 4 at position $21^{18}$. The protein was first described by Maroteaux et al. in $1988^{19}$ studying the electric organ of Torpedo californica. They already showed high similarities to a 140 amino acid sequence in a cDNA library of rat brain. The name synuclein was derived from its apparent localization in the presynaptic nerve ends and the nucleus. Very quickly a homologue was purified and characterized from bovine brain ${ }^{20,21}$ only occurring in synapses around neuronal cells and not in glial cells.

Ueda et al. ${ }^{22}$ then established the first link to neurodegeneration. They identified two short peptide fragments $X$ and $Y\left(X: N-{ }_{61} E_{\text {EQVTNVGGAVVTGVTAVAQK }}{ }_{80}-\mathrm{C}, \mathrm{Y}: \mathrm{N}-\right.$ ${ }_{81}$ TVEGAGSTAAATGFV $V_{95}-\mathrm{C}$ ) in purified amyloids from the frontal cortex of an $A D$ patient. With the sequence information of the peptides Ueda et al. were able to amplify the full-length aSyn DNA sequence from a human fetal brain cDNA library. The region spanned by the two fragments is therefore called non-A $\beta$ component (NAC). Jakes et al. ${ }^{23}$ and $\mathrm{Ji}$ et al. ${ }^{24}$ completed the synuclein family by identifying $\beta$ - and $\gamma$-synuclein respectively. $\beta$ - and $\gamma$-synuclein are lacking the NAC region and are not known to form amyloids in vivo. There are two relevant point mutations of $\beta$-synuclein that are associated with dementia with Lewy bodies and might be disease relevant ${ }^{25,26}$.

\subsubsection{Structure}

Under native functional conditions aSyn is considered to be a monomeric intrinsically disordered protein (IDP) ${ }^{27,28,29}$. The protein however maintains long-range tertiary interactions $^{30,31}$ and higher compactness than a random coil ensemble. On the sequence level, the protein can be divided into three regions, an amphipathic $\mathrm{N}$ terminus (residues 1-60), a hydrophobic central region (NAC region, residues 61-95) and an acidic C-terminal region (residues 96-140) that is rich in prolines (Figure 1-2). Although aSyn is highly dynamic in solution and can exchange between a large number of conformations, the protein acquires $\alpha$-helical secondary structure upon interaction with phospholipid bilayers and micelles mainly driven by the amphipathic $\mathrm{N}$-terminus. In the case of SDS micelles aSyn forms a bent-helix ${ }^{32}$ (Figure 1-2b), whereas with phospholipid bilayers it forms an extended $\alpha$-helix ${ }^{33}$ orchestrating the curvature of biological membranes. 
a

b

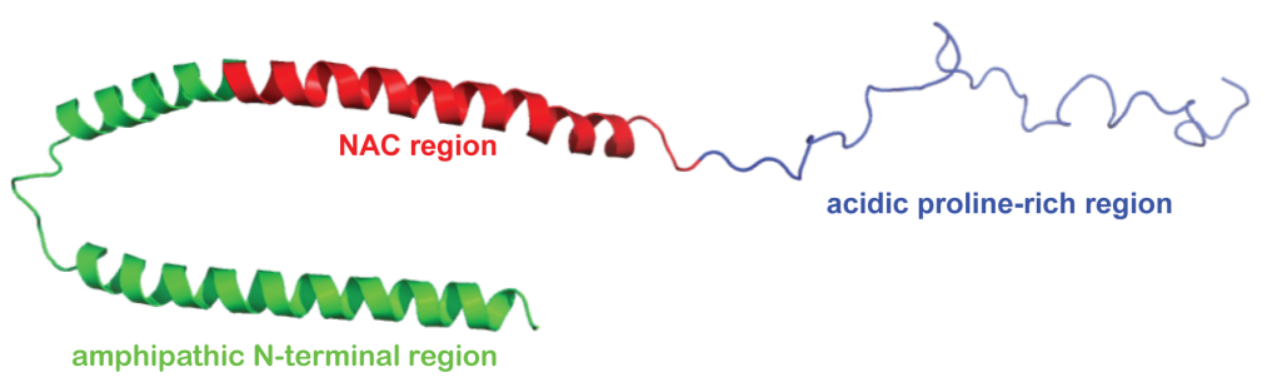

Figure 1-2. aSyn sequence and structural properties.

(a) aSyn sequence, amphipathic N-terminal region underlined in green, NAC region in red and acidic proline-rich region in blue. In orange mutations associated with familial forms of synucleinopathies are indicated: A30P, E46K, H50Q, G51D, A53E and A53T. The proline residues are indicated by bold letters, the KTKEGV-motif of the seven 11-residue imperfect repeat regions is shown in cyan. (b) Cartoon representation of aSyn bound to SDS micelles, curved $\alpha$-helix V3-V37 and K45-T92, PDB: 1XQ8.

Of special importance in the context of synucleinopathies, aSyn can also form amyloid fibrils ${ }^{4}$. Amyloid fibrils are highly structured and thermodynamically stable ${ }^{34}$ protein assemblies, that consist of intermolecular $\beta$-strands of polypeptide chains arranged perpendicular to the fibril axis and stacked by hydrogen bonding with a spacing of $4.7 \AA^{35}$, this arrangement is also named the cross- $\beta$ structure ${ }^{36,37}$. In in vitro studies the interconversion of aSyn into amyloid fibrils has been extensively studied using a wide variety of aggregation conditions with varying $\mathrm{pH}^{38}$, ionic strength, temperature ${ }^{39}$, concentration, additives ${ }^{40}$ and stimulation procedures. Figure 1-3a summarizes the different structural steps. Monomeric aSyn is converted into mature fibrils, also called preformed fibrils (PFF), involving the formation of transient on-pathway oligomers ${ }^{41}$ with $\beta$-sheet content. In vivo it is much more challenging to follow aSyn aggregation over time (Figure 1-3b). Physiological monomeric aSyn can be detected in the terminal ends of synapses. Granular structures might represent oligomeric or early fibrillar states of synuclein based on cell culture experiments, whereas complex structures 
such as pale bodies and Lewy bodies can only be observed in patients, however a simplified mouse model for pale bodies exists ${ }^{42}$.



Figure 1-3. Aggregation of aSyn in vitro and in vivo.

(a) In vitro aggregation scheme of monomeric aSyn. (b) Detectable morphological species in vivo by histological antibody staining. Figure adapted from these publications ${ }^{43,44,45}$.

\subsubsection{Functional aspects}

The $\alpha$-helical state of aSyn has been associated with its potential biological function in the presynaptic terminals that is until today only poorly understood. There is evidence, that aSyn-membrane interactions are crucial in synaptic transmission ${ }^{46}$ as well as the cycling of synaptic vesicles via endocytosis ${ }^{47}$. An important finding potentially related to the biological function of aSyn was obtained by George et al. ${ }^{48}$ on the bird species zebra finch, where synuclein mRNA and protein expression levels are downregulated over a period during development when the birds develop their final adult song, while the levels stay low during adult life in comparison to brain regions not involved in song control. The authors correlated their findings with neuronal plasticity. Directly related to aSyn's function in the presynaptic terminals is its role as a member in the assembly of the SNARE-complex, where it acts as a noncanonical chaperone ${ }^{49}$. This interaction is believed to happen through protein-protein interactions of aSyn's acidic C-terminus with synaptobrevin-2/VAMP2 while interacting with phospholipid bilayers of biological membranes through its $\mathrm{N}$-terminus ${ }^{50}$. Burré et al. ${ }^{50}$ also showed that aSyn undergoes oligomerization bound to membranes and that this step is crucial for the supposed chaperone function in membrane fusion and exocytosis. 


\subsection{Synucleinopathies}

The term $\alpha$-synucleinopathies or also just synucleinopathies defines all members of a family of proteinopathies sharing as a common feature intracellular protein aggregates of aSyn ${ }^{51,52}$ in neuronal and/or glial cells. There are two major pathologies: on one hand Lewy-related $\alpha$-synuclein pathologies (LRP) comprising Lewy bodies and Lewy neurites and on the other hand glial cytoplasmatic inclusions $(\mathrm{GCl})^{53,54}$. Lewy $\mathrm{FH}$ was the first to describe these inclusions in brain sections of PD patients in 1912 and Tretiakoff $\mathrm{C}$ later on in 1919 proved their presence in the substantia nigra ${ }^{55}$ naming these structures in Lewy's honor. Another key finding is the presence of Lewy bodies in the cortex ${ }^{56}$. Pale bodies are considered precursors of Lewy bodies ${ }^{57}$.

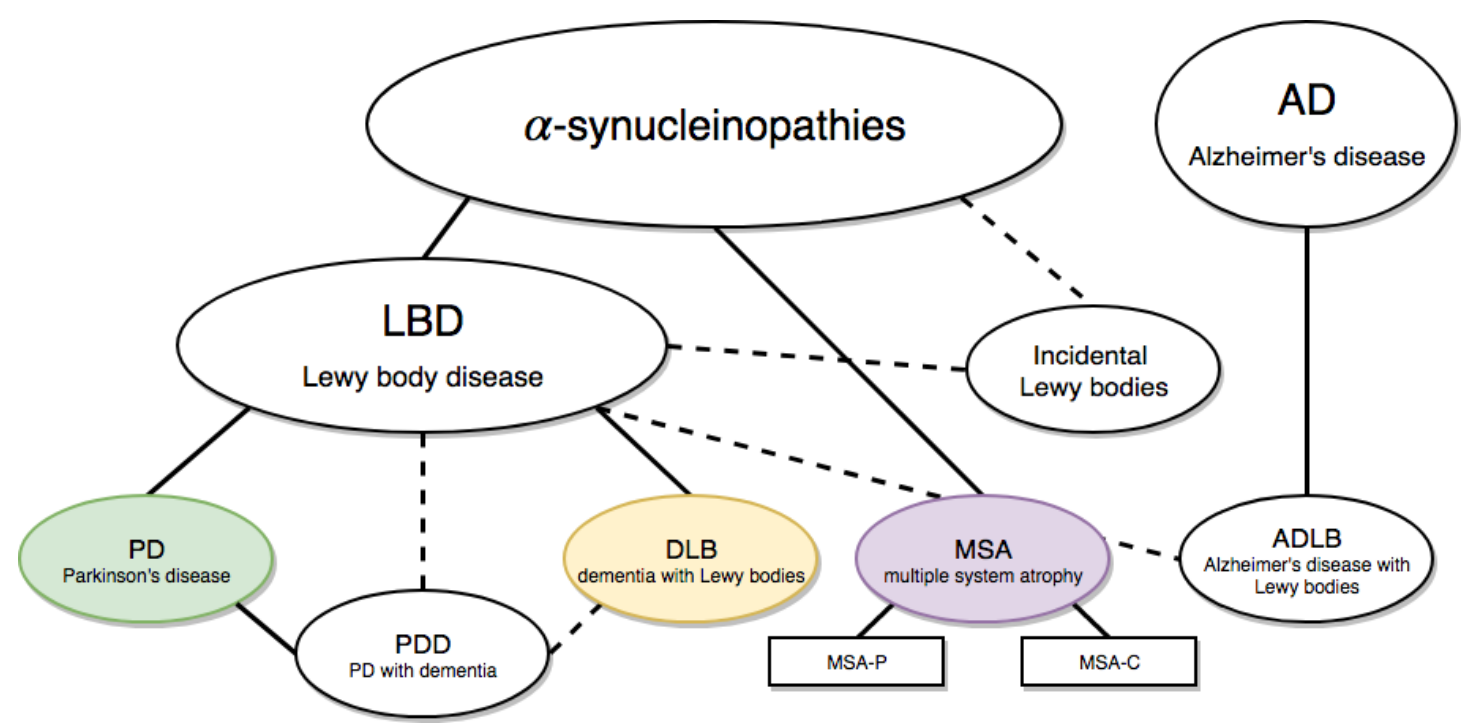

Figure 1-4. Preliminary classification of synucleinopathies.

Preliminary classification of synucleinopathies and associated diseases using information provided by Alafuzoff et al..$^{58}$ and McCann et al. ${ }^{59}$.

Lewy body composition has been in the focus of research over many years and fibrillar forms of aSyn have been identified as the key element often associated with posttranslational modifications such as phosphorylation ${ }^{60}$, mainly residue $\mathrm{S} 129$, as well as high levels of aSyn ubiquitination ${ }^{61}$ and others. In fact anti-ubiquitin antibodies were used for Lewy body staining before knowing about aSyn's role in synucleinopathies ${ }^{4}$. Importantly Lewy bodies are very diverse in their composition and should not be seen as pure inclusions of fibrillar synuclein. Indeed, more than 90 molecular components ${ }^{62}$ have been identified and some studies identified even more possible candidates ( $\sim 00$ proteins ${ }^{63}$ ), and similar findings have been reported for aSyn inclusions in animal 
model systems ${ }^{42}$. To the present day, it is not clear how LRPs and GCls are associated with toxicity and cell death.

The best medical diagnosis of synucleinopathies still relies on the postmortem examination of defined brain areas using immunohistochemical techniques. Figure 1-4 shows the different diseases in a hierarchic chart.

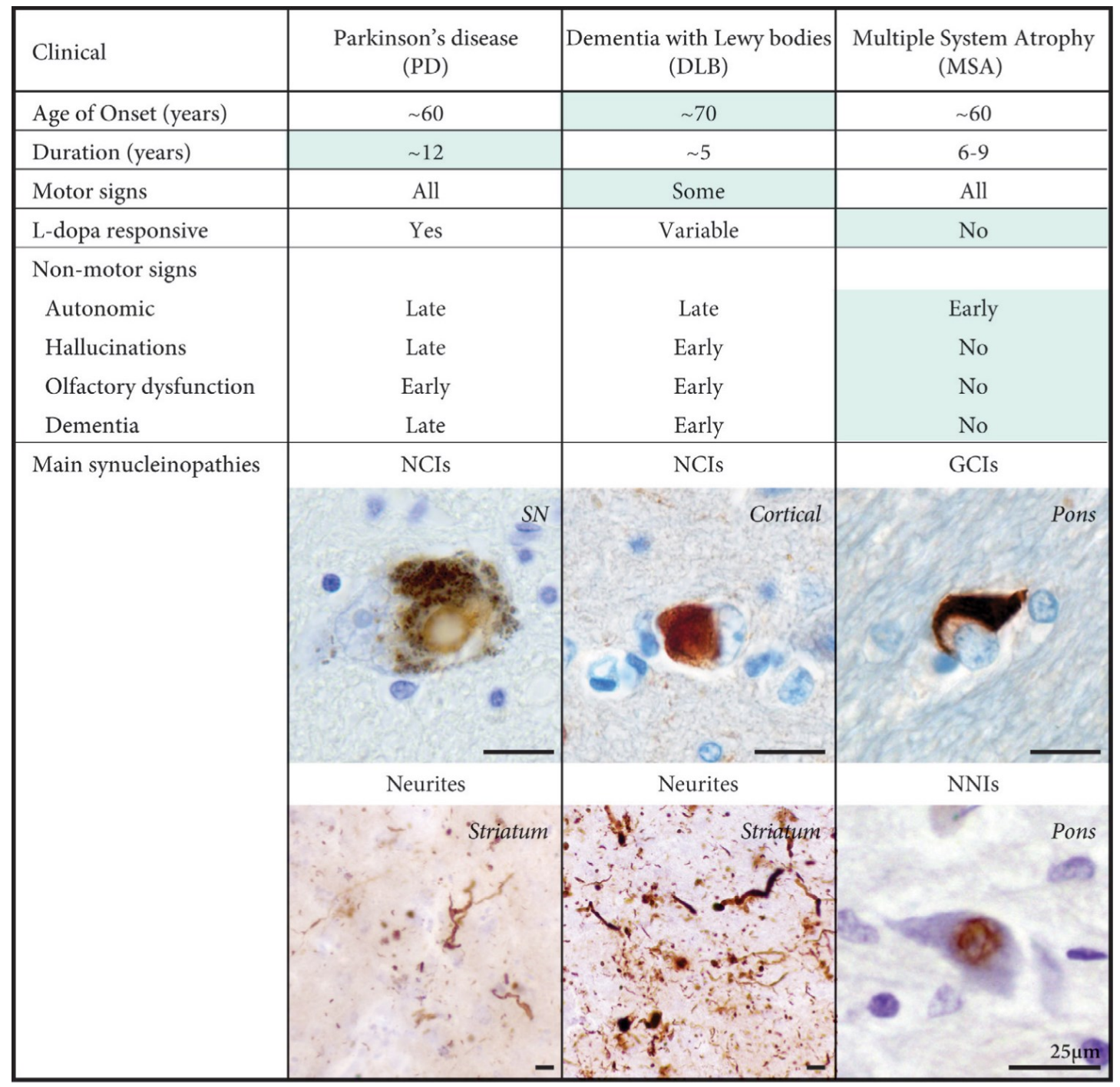

Figure 1-5. Hallmark pathologic features of PD, DLB and MSA.

Parkinson's disease (PD), Dementia with Lewy bodies (DLB) and Multiple System Atrophy (MSA). NCl: neuronal cytoplasmatic inclusions, GCl: glial cytoplasmatic inclusions, $\mathrm{SN}$ : substantia nigra. In green distinct pathologic features are indicated. Taken from McCann et al. ${ }^{59}$ 


\subsubsection{Parkinson's disease}

Parkinson's disease (PD) is the second most common age-related neurodegenerative disorder ${ }^{64}$ after Alzheimer's disease (AD) affecting 950 individuals per 100,000 in the $U^{65}$ which translates approximately in a prevalence rate of $1 \%$ in the Western world. A European prevalence study suggests that by the year 2030 between 8.7 and 9.3 million people will be affected by $\mathrm{PD}^{66}$.

The main clinical criteria for PD diagnosis is the presence of two out of three motor signs: bradykinesia, rigidity and resting tremor ${ }^{67}$. In addition, non-motor symptoms such as sensory impairments, REM sleep disorders, depression, constipation, pain as well as cognitive deterioration can occur, often becoming noticeable before the onset of motor symptoms.

The mutations $\mathrm{A} 30 \mathrm{P}^{68}, \mathrm{E} 46 \mathrm{~K}^{69}, \mathrm{H} 50 \mathrm{Q}^{70}, \mathrm{G}^{6} \mathrm{D}^{71}, \mathrm{~A}^{6} \mathrm{~T}^{72}$ and $\mathrm{A} 53 \mathrm{E}^{73}$ of aSyn are associated with familial forms of PD, as well as SNCA gene duplication ${ }^{74}$ and triplication ${ }^{75}$. Most of PD cases are sporadic. Key PD symptoms are directly linked to cell death of pigmented dopaminergic neurons in substantia nigra and are signs of dopamine deficiency. Post-mortem LPR in SN are detectable. Depigmentation of the substantia nigra can be readily observed in brain autopsy. Some PD patients also develop dementia and for some pathologists it is even classified as a distinct Lewy body disease, Parkinson's disease with dementia (PDD) (Figure 1-4). LPRs do not differ much between PD, PDD and LBD. For correct assessment of the disease type, it is absolutely crucial that in case of PD and PDD, signs of dementia start after the advent of motor symptoms and vice versa of DLB ${ }^{59}$.

Besides symptomatic pharmacological therapy of $\mathrm{PD}^{76}$ by L-DOPA and surgical deep brain stimulation ${ }^{77}$, there is down to the present day no cure for the diseases. Thus, further fundamental research is required, in order to better understand and characterize the disease and eventually help the millions of people affected.

\subsubsection{Dementia with Lewy bodies}

Dementia with Lewy bodies overlaps closely in its pathology with PD. It follows Alzheimer's disease as the most common form of dementia ${ }^{78}$. The worsening of cognitive functions associated with dementia has to precede motor symptoms for at least one year. LBP seems to be more pronounced in the cerebral cortex in comparison to PD (Figure 1-5). 


\subsubsection{Multiple system atrophy}

Multiple system atrophy (MSA) shows two disease subtypes. The first subtype named MSA-P is associated with classical symptoms also occurring in PD such as bradykinesia, tremor, rigidity and postural instability. The second subtype MSA-C affects mainly the cerebellum and the associated functions causing gait and speech problems as well as oculomotor dysfunctions ${ }^{79}$. MSA has a similar onset age as PD, but the survival time is much shorter with patients dying within 6-9 years. ${ }^{59}$ The most striking difference appears in the pathology, in MSA unlike any other synucleinopathy, the affected cells are non-neuronal oligodendrocytes of the brain glia and the hallmark feature are aSyn-positive glial cytoplasmatic inclusions (GCI) (Figure 1-5). Currently it is still an open question if the misfolded aSyn is expressed by the oligodendrocytes themselves ${ }^{80}$ or if there is a transmission pathway that allows transport of aSyn from neurons to glial cells $\mathbf{s}^{81}$ and equally important what is the conformation of the aSyn species that occur in this neuron-to-glia transmission.

\subsubsection{ADLB and others}

Lewy body pathology has also been observed in a subpopulation of patients diagnosed with Alzheimer's disease, specially within the amygdala ${ }^{82}$. These cases might represent a distinct pathologic entity or represent mixed-type pathologies of DLB and $A D^{83}$, making it difficult to categorize unanimously. In addition, it is known that Lewy bodies occur in asymptomatic patients, which is referred to as "incidental Lewy bodies" in the field. These cases could also represent preclinical stages of true synucleinopathies.

\subsection{Prion hypothesis and prion-like behavior of aSyn}

\subsubsection{Prion disease}

The native prion protein $\left(\mathrm{PrP}^{\mathrm{c}}\right)$ has in humans a length of 253 amino acids ${ }^{84}$ and is expressed in vertebrates ${ }^{85}$. The structure of $\operatorname{PrP}^{c}$ is known for mice ${ }^{86}$ and sheep ${ }^{87}$, it contains a globular $\mathrm{C}$-terminal domain comprised of three $\alpha$-helices and an intrinsically disordered $\mathrm{N}$-terminus. Native $\mathrm{PrP}^{\mathrm{c}}$ can misfold in vitro and in vivo in a $\beta$-sheet rich confirmation forming amyloid fibrils. The misfolded and infectious form of the protein is referred to as $\mathrm{PrP}^{\mathrm{Sc}}$, named after the neurodegenerative disease it causes in sheep, scrapie disease. The exact conformation of $\mathrm{PrP}^{\mathrm{sc}}$ fibrils is still unknown, but it lacks the $\alpha$-helical moiety of its native monomeric form ${ }^{88}$. $\operatorname{PrP}^{c}$ can spontaneously change its conformation and convert de novo into $\mathrm{PrP}^{\mathrm{Sc}}$. $\mathrm{PrP}^{\mathrm{Sc}}$ has autocatalytic activity and 
converts monomeric $\mathrm{PrP}^{\mathrm{c}}$ into $\mathrm{PrP}^{\mathrm{Sc}}$, also referred to as templated seeding. In humans it causes Creutzfeldt-Jakob disease (CJD) in two variants with distinct pathology ${ }^{89}$, sporadic (sCJD) and familial Creutzfeldt-Jakob disease (fCJD) $)^{90}$ associated with mutations in the prion gene (Prnp).

All prion diseases are transmissible, they are truly communicable ${ }^{91}$. In case of CJD the only reported transmissions were based on the usage of prion-contaminated hormone preparations from human cadavers ${ }^{92}$. There is a third form of CJD, the so called variant CJD (VCJD) that happens to cross-species infection of the cattle prion disease, bovine spongiform encephalopathy (BSE), by consumption of contaminated beef ${ }^{93}$. Genetically distinct $\mathrm{PrP}^{\mathrm{Sc}}$ of cattle causes the aggregation of human $\mathrm{PrP}^{\mathrm{c}}$ into $\mathrm{PrP}^{\mathrm{sc}}$. The prions of VCJD are biochemically distinct and show a different pathology with increased contagiousness. Transmission among humans via blood transfusions is possible and they conserve the characteristics of $\mathrm{vCJD}^{94}$. They are considered a prion strain and differences in pathology seem to be conformationally encoded ${ }^{15,95}$ (Figure 1-6).



Figure 1-6. Prion disease strain behavior.

Structurally and pathologically distinct population of prion strains causing different prion transmissible diseases. Taken from Tamgüney et al. ${ }^{96}$

\subsubsection{Protein misfolding and seeded aggregation}

The conversion of monomeric aSyn in the absence of fibrils or oligomers is called de novo aggregation. Hashimoto et al. ${ }^{97}$ reported for the first time that recombinant aSyn aggregates in vitro into amyloid fibrils (Figure 1-3). Wood et al. ${ }^{98}$ shortly after showed that the aggregation could be speeded up by the addition of preformed fibrils obtained by de novo aggregation. This opened the field for in depth biophysical investigations of aSyn aggregation and the aggregation kinetics, as well as variants of aSyn associated with familial mutations ${ }^{99}$. It is important to point out that aSyn under quiescent conditions does not form fibrils at $37^{\circ} \mathrm{C}$ and $\mathrm{pH} 7.4$ and stays in solution, shown in detail by Buell et al. ${ }^{100}$. The same group also showed, that the addition of seeds under quiescent conditions leads to fibril elongation, excluding secondary 
nucleation processes. In the prion field two techniques were developed to amplify prion strains by converting recombinant $\mathrm{PrP}^{\mathrm{C}}$ into $\mathrm{PrP}^{\mathrm{Sc}}$ with seeds from cellular material ${ }^{101}$ named real-time quaking induced conversion ${ }^{102}$ (RT-QuIC) and protein misfolding cyclic amplification ${ }^{103}$ (PMCA). Both techniques are now established in the synuclein field. RT-QuIC is thoroughly tested on cerebral spine fluid (CSF) of patients ${ }^{104}$ for diagnosis purposes. Herva et al. recently improved the PMCA and showed that PMCA end products are bona fide synuclein amyloid fibrils maintaining the structural properties ${ }^{105}$. Jung et al. used brain homogenates of pathologic tissues ${ }^{106}$ and also independently showed conservation of structural properties.

\subsubsection{Spreading in synucleinopathies}

Braak et al. introduced in a milestone research a staging system for AD pathology ${ }^{107}$ and applied the methodology later on to $\mathrm{PD}^{108}$, correcting recently some assumptions on the initial origin in the early phases of the disease ${ }^{109}$. There is also an alternative staging system by McKeith et al. ${ }^{110}$ and in addition, Beach et al. proposed modifications to Braak's stages ${ }^{111}$.

Stage $1 / 2$


Stage $3 / 4$


Stage $5 / 6$


Figure 1-7. Braak's staging of PD pathology.

Arrows indicate temporal disease progression in the central nervous system over anatomically interconnected brain regions, red color indicates severity of PD pathology. Key brain areas are listed in the upper panel. Taken from Goedert et al. ${ }^{112}$ 
Braak's disease progression model is based on the detailed analysis of individual postmortem brains of PD diagnosed patients, grouping their lesions due to severity and brain regions affected. He could thereby show, that all patients investigated follow a pattern that can be correlated with a temporal progression of $\mathrm{PD}$, starting in the brain stem. The open question remains if the pathology starts independently developing at different time points and/or different velocities or if the pathology travels along connected brain areas and ultimately spreads from cell-to-cell. Inevitably the question arises, what would be the nature of the "pathogen" and what are the routes along which the "pathogen" travels ${ }^{113}$. The best candidate for the pathogen is aSyn itself. For the spreading nature of pathology in neurodegenerative disorders caused by amyloidogenic proteins themselves, the term "prion-like" is established.

Pioneering work of two groups independently showed via postmortem analysis of PD brains, that Lewy body pathology can spread from neuronal tissue of the patients into the newly grafted tissue for patients that received fetal tissue transplants and survived for less than twenty years ${ }^{114,115}$.

A plethora of animal model studies, see Table 1 of Steiner et. $\mathrm{al}^{116}$, were performed where in vitro aggregated recombinant aSyn fibrils under different aggregation conditions were applied via intracranial injection into specific brain areas (e.g. striatum or substantia nigra) of the animal models of choice, mainly in rodent models. Afterwards the affected brain area and neighboring regions were thoroughly investigated and spreading of pathology into distal areas were consistently shown. Thanks to these experimental studies, we have strong evidence that aSyn fibrils get internalized by neuronal cells in vivo and stimulate LB-like pathology and neurotoxicity. Internalized aSyn fibrils also seem to stimulate the aggregation of endogenously expressed $a \mathrm{aSyn}^{117}$. Some of these experiments however fall short on answering the burning question of cell-to-cell transmission unanimously. Due to the experimental design and the intrinsic limitations of the approach, it cannot be always ruled out that aSyn pathology is induced due to internalization of exogenous injected material in distal areas to the injection site. If not, pathology in distal areas could only stem from de novo pathology close to the site of injection and would imply true cell-to-cell transmission ${ }^{116,118}$. A recent study by Rey et al. ${ }^{119}$ seems to rule out this issue by applying PFF to the olfactory bulb of a mouse model and observing widespread aSyn pathology in distant brain regions. In conclusion, there is strong evidence for prion-like cell-to-cell transmission in synucleinopathies and a similar picture evolves for other neurodegenerative disorders associated with $A \beta$, tau and huntingtin ${ }^{120}$. 
1.4.4 Prion strain-like behavior in synucleinopathies and structural polymorphism In 2013, Guo et al. ${ }^{121}$ showed that in vitro aSyn fibrils obtained through de novo aggregation (strain $A$ ) differ from in vitro fibrils that were obtained through repetitive seeding (strain B) and show distinct pathology in primary neuron cultures upon fibril transduction. Guo et al. worked on a C-terminally truncated aSyn construct only comprising residues 1-120 and bearing a C-terminal Myc tag and not wt aSyn. Strain $A$ and $B$ also differed in a proteinase $K$ digestion assay, indicating the presence of conformational differences. In addition, Bousset et al. ${ }^{122}$ characterized two in vitro aSyn fibril polymorphs that were obtained under high- $(50 \mathrm{mM}$ Tris- $\mathrm{HCl}, 150 \mathrm{mM} \mathrm{KCl}, \mathrm{pH}$ 7.5) and low (5 mM Tris- $\mathrm{HCl}, \mathrm{pH} 7.5)$ salt conditions using a de novo aggregation approach. Solid-state NMR spectroscopy showed that these two types of in vitro fibrils differ structurally with additional $\beta$-structure present in the $\mathrm{N}$-terminal region of the low salt polymorph. Bousset et al. also showed, that the high and low salt polymorph of aSyn (hsAsyn and IsAsyn respectively) cause different toxicities in a neuronal cell line upon transduction. In a follow-up publication by Peelaerts et al. ${ }^{117}$, the two polymorphs were shown to result in distinct pathology in a rat animal model (Wistar rats). In addition, the proteinase $\mathrm{K}$ digestion suggested that the confirmation of the polymorphs was conserved throughout in vivo passaging. Thus, increasing evidence suggests that aSyn not only leads to templated seeding in vivo and in vitro, but it also conserves structural features of the primary seed summed up under the term imprinting. The term prion-like seems to be therefore justified, because a true prion behavior with disease transmission among humans has not been observed ${ }^{123}$ yet.

Because these findings were obtained using in vitro prepared fibrils, it remains to be shown if strain-like behavior also occurs in patient brains and if differences in pathology among the distinct synucleinopathies are associated with conformationally distinct aSyn strains? To address the question, Prusiner et al. ${ }^{124}$. used brain homogenates of 14 postmortem brains of patients diagnosed with multiple system atrophy (MSA) and Parkinson's disease (PD), six PD cases included in the study, in combination with a hemizygous mouse model (TgM83+/-) that overexpresses human full-length A53T aSyn protein. The mice were intracerebrally injected with brain homogenate and 120 days post-injection the animals were sacrificed and the brains analyzed for neuropathology. Only brain homogenate of MSA patients was able to induce neuropathology with atypical Lewy body pathology in neurons. MSA-infected mouse brain homogenate can also induce pathology upon reapplication to a new TgM83+/- cohort. PD brain homogenate in contrast did not cause neurodegeneration in the mouse model, neither in the first generation with human brain homogenate nor 
in the following generation with homogenized mouse brain. This provides further support for the strain model of synucleinopathies (Figure 1-8).



Figure 1-8. Strain behavior in neurodegenerative diseases.

General scheme of strain behavior in neurodegenerative diseases, taken from Walker LC ${ }^{15}$.

Prusiner et al. also report that MSA as well as PD brain homogenate failed to induced pathology in mice expressing human or mouse wt aSyn. A similar study by Bernis et al. ${ }^{125}$ showed neuropathology in mice expressing $w t$ human aSyn for brain homogenate of MSA patients and patients with incidental Lewy body pathology, which questioning the unique role of aSyn strains in MSA.

\subsubsection{Fluorescent dyes as molecular probes for disease-specific conformations}

The binding of fluorescent dyes to amyloid structure is well established and used to stain protein deposits in tissue ${ }^{126,127}$. The Congo red derivative FSB binds amyloid plaques of $A \beta$ as well as NTFs of tau protein ${ }^{128,129}$. FSB and Curcumin also allowed determination of intersubject variability of amyloid- $\beta$ aggregates in Alzheimer's disease ${ }^{130}$, while fluorescent polythiophene dyes were used to distinguish prion strains $^{131}$. The polythiophene derivative HS-68 was developed to successfully probe differences between NTFs and amyloid plaques ${ }^{132}$.

\subsection{High resolution structures of in vitro aSyn fibrils}

The first high resolution structure of human wt aSyn in vitro fibrils was solved by Tuttle et al. ${ }^{133}$ using solid-state NMR (PDB accession code: 2 NOA). Tuttle et al. showed that the monomeric aSyn entity of an aSyn fibril under their in vitro aggregation condition forms a Greek key fold (Figure 1-9c) stabilized by hydrogen bonds in register along the fibril axis. The fibrils were de novo aggregated in a sodium buffer (50 mM NaP, pH 7.4, 0.1 mM EDTA, $0.02 \% \mathrm{NaN}_{3}$ ). 
In July 2018 two groups almost simultaneously published cryo-electron microscopy structures of in vitro aSyn fibrils (Figure 1-9 a, b). Guerrero-Ferreira et al. ${ }^{134}$ aggregated a C-terminally truncated construct of aSyn (residues 1-121) in Dulbecco's phosphate-buffered saline (DPBS: $2.66 \mathrm{mM} \mathrm{KCl}, 1.47 \mathrm{mM} \mathrm{KH}_{2} \mathrm{PO}_{4}, 137.93 \mathrm{mM} \mathrm{NaCl}$, $8.06 \mathrm{mM} \mathrm{Na}_{2} \mathrm{HPO}_{4}, \mathrm{pH}$ 7.0-7.3). They were able to confirm the Greek key topology, but in contrast to Tuttle et al. the aSyn fibrils consist of two entangled protofibrils that form a protofibril interface from residue 50 to 57 . The group was able to model aSyn residues 38 to 95 into the electron density map (PDB accession code: $6 \mathrm{H6B}$ ) at a resolution of $3.4 \AA$ (Figure 1-9a). For the missing residues no electron density was observed, indicating that those residues are not part of the fibril core and show high conformational flexibility in the absence of intermolecular hydrogen bonds.

$\mathrm{Li}$ et al. ${ }^{135}$ determined the structure of another aSyn in vitro fibril by cryo-EM. Full-length wt aSyn was fibrillated under identical high salt conditions as in Bousset et al. ${ }^{122}$ and Peelaerts et al. ${ }^{117}$. The structure of Li et al. thus provides a high-resolution view of the "high salt polymorph" (hsAsyn) investigated by Bousset et al. and Peelaerts et al.. Li et al. deposited a structure (PDB accession code: 6A6B) comprising residues 37 to 99 at a resolution of $3.07 \AA$ (Figure 1-9b). The structure of a single aSyn molecule is highly similar for residues 38-95 in the cryo-EM structures of Guerrero-Ferreira et al. and Li et al. and also closely overlaps with the Greek key topology determined by solidstate $\mathrm{NMR}^{133}$. In addition, the structure of Li et al. displays the dimer of two protofibrils with an almost identical protofilament interface ranging from residue 50 to 57 . The two deposited cryo-EM structures, however, differ on the quaternary level. Li et al.'s structure is left-handed while Guerrero et al.'s structure right-handed. These differences could be due to the two groups using different aSyn constructs and/or differences in aggregation buffers. 
a

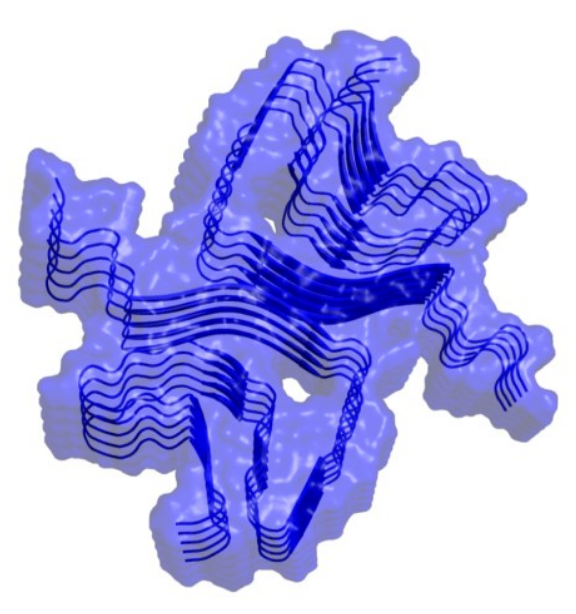

C

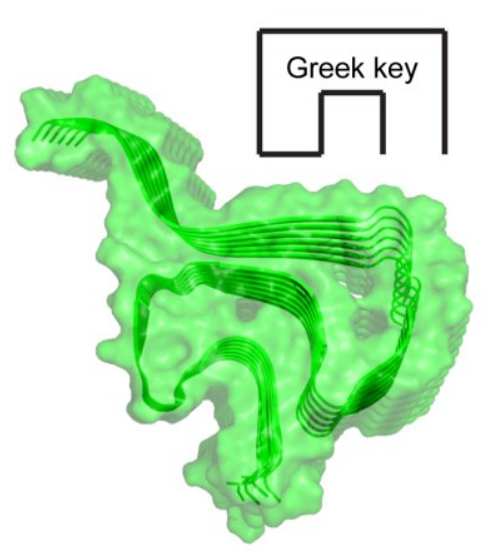

b

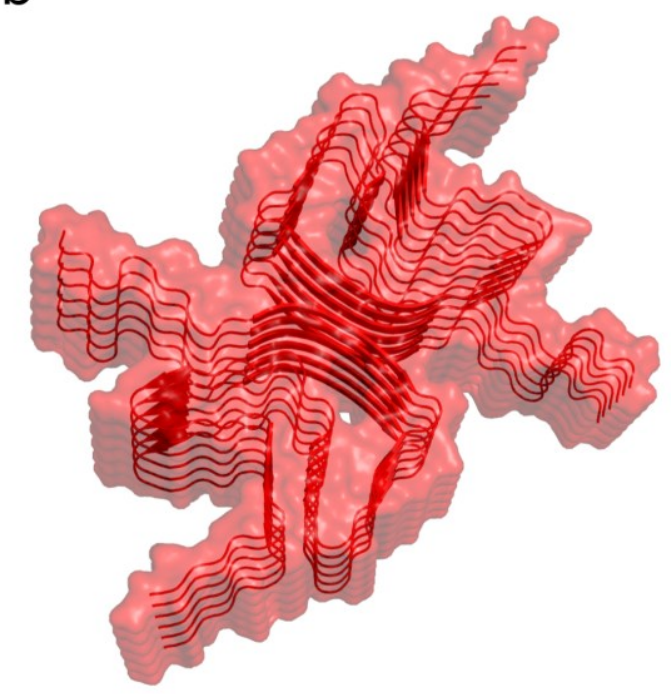

d

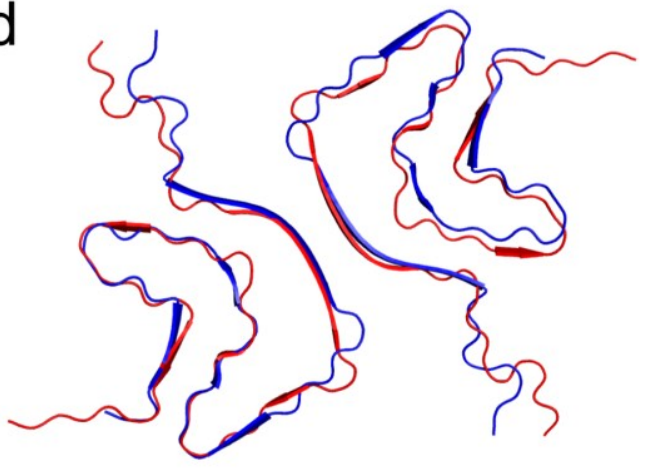

Figure 1-9. High resolution structures of in vitro aSyn fibrils.

(a) In blue cryo-EM structure of Guerrero-Ferreira et al. (July 3, 2018), PDB-code: 6H6B, residue 38-95. (b) In red cryo-EM structure of Li et al. (July 31, 2018), PDB: 6A6B, residue 3799. (c) Solid-state NMR structure of Tuttle et al. (2016), PDB: 2NOA, residue 37-99. (d) Planar overlay of cryo-EM structures blue $(6 \mathrm{H} 6 \mathrm{~B})$ and in red $(6 \mathrm{~A} 6 \mathrm{~B})$.

\subsection{Aims and outline}

In the field of neurodegenerative diseases, and in particular for synucleinopathies, the paradigm has evolved that these diseases are caused by prion-like misfolded protein aggregation and spreading of pathology through the nervous system. On the other hand, there is striking evidence that $\alpha$-synuclein fibrils generated in vitro under different aggregation conditions show conformational diversity, so called "polymorphs", associated with distinct toxicities in cells and animal models.

Since synucleinopathies comprise a broad spectrum of pathologies and symptoms that cluster in distinguishable disease types with striking differences in the 
manifestation of the disease on the molecular level, the temptation of merging the two observations into one single scientific concept, the strain-like behavior of $\alpha$-synuclein in synucleinopathies, is high and lively debated in the field. However, the final cornerstone is missing and as convincing as the concept appears, further experimental evidence is needed to proof the hypothesis.

The work of this thesis directly aims to address $\alpha$-synuclein's potential strainlike behavior in synucleinopathies. To this end, the project followed a clear strategy:

1. The amplification of disease-relevant $\alpha$-synuclein fibrils from patient brains of distinct synucleinopathies (PD, MDA and DLB) through PMCA by collaborators.

2. The setup of a hydrogen-deuterium (HD) exchange coupled to NMR spectroscopy pipeline based on an established protocol using two well characterized in vitro $\alpha$-synuclein polymorphs achieving single-residue resolution.

3. Biophysical characterization of $\alpha$-synuclein fibrils for both in vitro $\alpha$-synuclein fibrils as well as for $\alpha$-synuclein fibrils amplified from patients' brains by negative-stain EM, CD and fluorescent dye binding.

4. Correlation of single-residue HD exchange results by NMR for the high and low salt polymorphs with available data in literature. In addition, for the high salt polymorph mapping of HD exchange data to the available high-resolution cryoEM structure.

5. Inter-disease correlation of HD exchange data and mapping to high-resolution cryo-EM structure.

6. Characterization of $\alpha$-synuclein fibrils by ortholog high-resolution EPR spectroscopy through collaboration. 


\section{Materials and methods}

\subsection{Chemical reagents}

(E,E)-1-fluoro-2,5-bis(3-hydroxycarbonyl-4-hydroxy)styrylbenzene (FSB) from Santa Cruz Biotechnology (Lot \# D1012, sc-359845), Curcumin from Sigma-Aldrich (Lot \# WXBC5818V, 08511-10MG), HS-68 synthesized by the Synthetic Chemistry Facility of the MPIBPC Göttingen, (1-oxy-2,2,5,5-tetramethyl-d-pyrroline-3-methyl)methanethiosulfonate (MTSL) from Toronto Research Chemicals, Ammonium chloride $\left({ }^{15} \mathrm{~N}, 99 \%\right)$ from Cambridge Isotope Laboratories, D-Glucose (U-13C6, 99\%) from Cambridge Isotope Laboratories, Deuterium oxide from Sigma-Aldrich (7568221.107KG), Guanidine thiocyanate (BioUltra, >99.0\%) from Sigma-Aldrich (50980).

\subsection{Bacterial strains and plasmids}

Cell lines: $\quad$ Competent BL21(DE3) cells purchased from Invitrogen (44-0048)

Plasmids: $\quad$ pT7-7 aSyn WT provided by the Lansbury Laboratory, Harvard Medical School, Cambridge

pNatB (pACYCduet-naa20-naa25) purchased from Addgene (53613)

\subsection{Equipment}

Bruker NMR spectrometers (400 - $950 \mathrm{MHz}$ ), Eppendorf Thermomixer comfort, Eppendorf Entris precision balance, IKA KS 4000i incubator, INFORS HT Multitron incubator, GFL water bath, AVESTIN EmulsiFlex C3 French press, Beckman Coulter centrifuge Avanti JXN-26, Beckman Coulter JLA-8.1 rotor, Beckman Coulter JA-25 rotor, Eppendorf Centrifuge 5810R, Eppendorf Centrifuge 5424R, GE Äkta prime plus, GE Äkta pure, Eppendorf BioSpectrometer, Beckman Coulter Optima MAX-XP Ultracentrifuge, Bandelin Sonorex Digitec ultrasonic bath, Agilent Technologies Cary Eclipse fluorescence spectrophotometer, Applied Photophysics qCD Chirascan spectrophotometer, Agilent Technologies 1260 Infinity HPLC pump and UV detection unit, Wyatt miniDAWM TREOS, Wyatt Optilab T-rEX, Wyatt DynaPro NanoStar, ThemoFisher Herasafe KS clean bench, Heidolph Duomax 1030 shaker, Sartorius CPA 2245 analytical balance, BioRad GelDoc XR+, TECAN Spark 20 M plate reader, ThermoScientific Heratherm incubator, Mono Q 5/50 GL, GE Superdex 75 and Superdex 200 16/600 GL, GE Superdex 75 and S200 26/600, GE Superdex 10/300 GL, Sharp microwave. 


\subsection{Software}

Topspin (Bruker), Sparkcontrol v2.2 (TECAN), Pro-Data Chirascan v4.5 (Applied Photophysics), NMRPipe ${ }^{136}$ v8.9, Prism v7 (GraphPad), Excel v16 (Microsoft), MATLAB R2016b (MathWorks), DeerAnalysis2018 ${ }^{137,138}$, CcpNmr Analysis ${ }^{139}$ v2.4, PyMOL v2.2.0, Illustrator CS v5.1 (Adobe), Word v16 (Microsoft), EndNote X8, Affinity Photo v1.6.

\subsection{Buffers}

Low salt buffer: $\quad 5 \mathrm{mM}$ Tris- $\mathrm{HCl}, \mathrm{pH} 7.5,0.02 \% \mathrm{NaN}_{3}$

High salt buffer: $\quad 50 \mathrm{mM}$ Tris- $\mathrm{HCl}, 150 \mathrm{mM} \mathrm{KCl}, \mathrm{pH} 7.5,0.02 \% \mathrm{NaN}_{3}$

HEPES buffer: $\quad 50 \mathrm{mM} \mathrm{HEPES}, 100 \mathrm{mM} \mathrm{NaCl}, \mathrm{pH} 7.4,0.02 \% \mathrm{NaN}_{3}$

\subsection{Protein preparation}

Purification was performed as previously described by Hoyer et al. ${ }^{38}$. If not explicitly stated otherwise, aSyn refers to $\mathrm{N}$-terminally acetylated $w t \alpha$-synuclein. Stefan Becker and Karin Giller (NMR2, Max-Planck-Institute for Biophysical Chemistry, Göttingen, Germany) kindly provided non-acetylated $w t \alpha$-synuclein for reference experiments, stock solution $0.3 \mathrm{mM}$ in HEPES buffer. Sol Cima (Translational Structural Biology in Dementia, DZNE, Göttingen, Germany) helped with the preparation of aSyn.

\subsubsection{Transformation and glycerol stock}

Human $\mathrm{N}$-terminally acetylated wt $\alpha$-synuclein (aSyn) was obtained by co-transfection of $E$. coli BL21 (DE3) cells with pT7-7 plasmid encoding for wt human $\alpha$-synuclein and NatB acetylase complex of Schizosaccharomyces pombe ${ }^{140}$ using pNatB plasmid. The pT7-7 plasmid was kindly provided by the Lansbury Laboratory, Harvard Medical School, Cambridge MA. The pNatB plasmid (pACYCduet-naa20-naa25) was a gift from Dan Mulvihill (Addgene plasmid \# 53613). BL21(DE3) Competent E. coli cells were thawed on ice, $300 \mathrm{ng}$ of each plasmid DNA was added and incubated for 5 minutes on ice. Heat shock was performed for $45 \mathrm{sec}$ at $42{ }^{\circ} \mathrm{C}$ on an Eppendorf Thermomixer comfort. $200 \mu \mathrm{L}$ of autoclaved LB medium was added and the temperature was set to $37^{\circ} \mathrm{C}$. The BL21 (DE3) cells were incubated for another 30 minutes under shaking, $650 \mathrm{rpm} .50 \mu \mathrm{L}$ of cells were plated out on LB-agar plates with $100 \mu \mathrm{g} / \mathrm{ml}$ ampicillin (Amp) and $25 \mu \mathrm{g} / \mathrm{ml}$ chloramphenicol (Chl), plates were incubated at $37^{\circ} \mathrm{C}$ o/n. A single E. coli colony was picked the following day and o/n culture grown 
in $7 \mathrm{ml} \mathrm{LB}$ medium $(100 \mu \mathrm{g} / \mathrm{ml} \mathrm{Amp,} 25 \mu \mathrm{g} / \mathrm{ml} \mathrm{Chl})$ at $37^{\circ} \mathrm{C}$. Eventually $500 \mu \mathrm{L}$ of o/n culture was mixed with $500 \mu \mathrm{L}$ of sterile $50 \%$ glycerol in water in a $2 \mathrm{ml}$ screw top tube, gently mixed and shock frozen in liquid nitrogen, storage at $-80^{\circ} \mathrm{C}$.

\subsubsection{Protein expression}

$25 \mathrm{ml}$ LB medium preculture $(100 \mu \mathrm{g} / \mathrm{ml} \mathrm{Amp,} 25 \mu \mathrm{g} / \mathrm{ml} \mathrm{Chl})$ was inoculated with BL21(DE3) pT7-7 pNatB glycerol stock and grown o/n at $37^{\circ} \mathrm{C}$. A sterile disposable inoculation loop was used to scratch the glycerol stock surface and then inserted into the LB medium of the preculture. For unlabeled aSyn expression, $2 \mathrm{ml}$ of o/n preculture was added to $1 \mathrm{I}$ of LB medium (Amp/Chl) and grown to $\mathrm{OD}_{600}$ of $\sim 0.8$, induced with $0.5 \mathrm{mM}$ IPTG final concentration and incubated at $25^{\circ} \mathrm{C} \mathrm{o} / \mathrm{n}$. For ${ }^{15} \mathrm{~N}$-labeled and double-labeled $\left({ }^{13} \mathrm{C}-{ }^{15} \mathrm{~N}\right.$-labeled) aSyn, the protocol was slightly adapted. $4 \mathrm{ml}$ of the $20 \mathrm{ml}$ o/n LB medium preculture was shortly centrifuged in a $15 \mathrm{ml}$ Falcon tube and the pellet resuspended in $4 \mathrm{ml} 1 \times \mathrm{M} 9$ minimal medium (Amp, Chl) before adding it to $1 \mathrm{I}$ of $1 \mathrm{x}$ M9 medium (Amp/Chl) supplemented with ${ }^{15} \mathrm{NH}_{4} \mathrm{Cl}(0.5 \mathrm{~g} / \mathrm{l})$ and in case of double-labeled supplemented additionally with $\mathrm{D}-{ }^{13} \mathrm{C}$-Glucose $(4 \mathrm{~g} / \mathrm{l})$ and further grown to $\mathrm{OD}_{600}$ of $\sim 0.8$. $0.5 \mathrm{mM}$ IPTG was added for induction and protein expression happened at $25^{\circ} \mathrm{Co} / \mathrm{n}$.

\begin{tabular}{ll}
\hline Compound & Amount \\
\hline M9 salts $(5 \mathrm{x})$ & $200 \mathrm{ml}$ \\
Trace elements & $10 \mathrm{ml}$ \\
$\mathrm{CaCl}_{2}(1 \mathrm{M})$ & $300 \mathrm{\mu l}$ \\
$\mathrm{MgSO}_{4}(1 \mathrm{M})$ & $1 \mathrm{ml}$ \\
Thiamine $(10 \mathrm{mg} / \mathrm{ml})$ & $0.1 \mathrm{ml}$ \\
$\mathrm{D}-\mathrm{Glucose} / \mathrm{D}-{ }^{13} \mathrm{C}-$ Glucose & $4 \mathrm{~g} / 4 \mathrm{~g}$ \\
${ }^{15} \mathrm{NH}_{4} \mathrm{Cl}$ & $0.5 \mathrm{~g}$ \\
$\mathrm{H}_{2} \mathrm{O}$ (deionized) & up to $\mathrm{V}_{\mathrm{t}}=1 \mathrm{l}$ \\
\hline
\end{tabular}

Table 2. Recipe for 1 I M9 minimal medium.

M9 salts (5x): $\quad 30 \mathrm{~g} \mathrm{Na}_{2} \mathrm{HPO}_{4}, 20 \mathrm{~g} \mathrm{KH}_{2} \mathrm{PO}_{4} \times 3 \mathrm{H}_{2} \mathrm{O}, 2.5 \mathrm{~g} \mathrm{NaCl}$ dissolved in $1 \mathrm{I} \mathrm{H}_{2} \mathrm{O}$ (deionized)

Trace elements: $\quad 0.6 \mathrm{FeSO}_{4} \times 7 \mathrm{H}_{2} \mathrm{O}, 0.115 \mathrm{~g} \mathrm{MnCl}_{2} \times 4 \mathrm{H}_{2} \mathrm{O}, 0.08 \mathrm{~g} \mathrm{CoCl}_{2} \times 6 \mathrm{H}_{2} \mathrm{O}$, $0.07 \mathrm{ZnSO}_{4} \times 7 \mathrm{H}_{2} \mathrm{O}, 0.03 \mathrm{CuCl}_{2} \times 2 \mathrm{H}_{2} \mathrm{O}, 0.002 \mathrm{~g} \mathrm{H}_{3} \mathrm{BO}_{3}, 0.025 \mathrm{~g}$ $\left(\mathrm{NH}_{4}\right)_{6} \mathrm{Mo}_{7} \mathrm{O}_{24} \times 4 \mathrm{H}_{2} \mathrm{O}, 0.5 \mathrm{~g}$ EDTA dissolved in $100 \mathrm{ml} \mathrm{H} \mathrm{H}_{2} \mathrm{O}$ (deionized) 


\subsubsection{Cell harvest and purification}

Cells were harvested at $4{ }^{\circ} \mathrm{C}$ for 15 minutes at $7000 \mathrm{rpm}$ using a Beckmann Coulter centrifuge with a JLA-8.1 rotor. The supernatant (SN) was discarded and the bacteria pellet stored for further use at $-20^{\circ} \mathrm{C}$. The cell pellets were resuspended in $20 \mathrm{ml}$ per 1 I culture volume lysis buffer (10 mM Tris- $\mathrm{HCl}$, pH 8, $1 \mathrm{mM}$ EDTA, 1mM PMSF). Cell lysis was achieved using a French press. The cell lysate was boiled in a water bath at $\sim 100{ }^{\circ} \mathrm{C}$ for 30 minutes and centrifuged in a JA-25 rotor at $13,500 \mathrm{rpm}$ at $4{ }^{\circ} \mathrm{C}$ for 30 minutes, the SN was saved. DNA of SN was precipitated by adding at a ratio of $1: 10$ streptomycin sulfate from a stock solution in water $(100 \mathrm{mg} / \mathrm{ml})$. The DNA precipitation was enhanced by constant shaking for 15 minutes at $4{ }^{\circ} \mathrm{C}$. The precipitated DNA was separated as a pellet from the solution by a 30-minute centrifugation step, using a JA25 rotor $\left(13,500 \mathrm{rpm}, 4^{\circ} \mathrm{C}\right)$. Thereafter aSyn and residual protein impurities were precipitated by adding ammonium sulfate, weighing in the salt to a final concentration of $360 \mathrm{mg} / \mathrm{ml}$ and incubating the solution for 15 minutes at $4{ }^{\circ} \mathrm{C}$ under constant shaking. The protein pellet was harvested by centrifugation as described earlier and the $\mathrm{SN}$ discarded. The pellet was either stored at $-20{ }^{\circ} \mathrm{C}$ or directly used for further purification steps.

The pellet was then resuspended in $25 \mathrm{mM}$ Tris-HCl buffer, $\mathrm{pH} 7.7$ and dialysed o/n against $4 \mathrm{~L}$ of the same buffer, Roche protease inhibitor added. The dialysate was sterile filtered and applied to a Mono Q 5/50 GL anion exchange column. To the column a salt gradient (25 mM Tris- $\mathrm{HCl}, \mathrm{pH} 7.7,0-1 \mathrm{M} \mathrm{NaCl}$ ) was applied, aSyn elutes around $300 \mathrm{mM} \mathrm{NaCl}$. The aSyn fraction was collected and loaded onto a Superdex gel filtration column (S75 and/or S200) in order to remove high and low weight impurities. Gel filtration was always performed in fresh, sterile filtered and degassed HEPES buffer. The final pure protein yield was concentrated via centrifugal concentrators (MWCO $5 \mathrm{kDa}$ ) and sterile filtered $(0.22 \mu \mathrm{m})$. Aliquots at concentrations between 500 $\mu \mathrm{M}$ and $1 \mathrm{mM}$ were shock-frozen in liquid nitrogen and stored at $-80^{\circ} \mathrm{C}$.

All steps of the purification were monitored by SDS-PAGE with $15 \%$ gels and the protein concentrations were determined by an Eppendorf BioSpectrometer using $100 \mu \mathrm{L}$ quartz cuvettes and an extinction coefficient of $5960 \mathrm{M}^{-1} \mathrm{~cm}^{-1}$. An example purification is shown in Figure 2-1. 
a
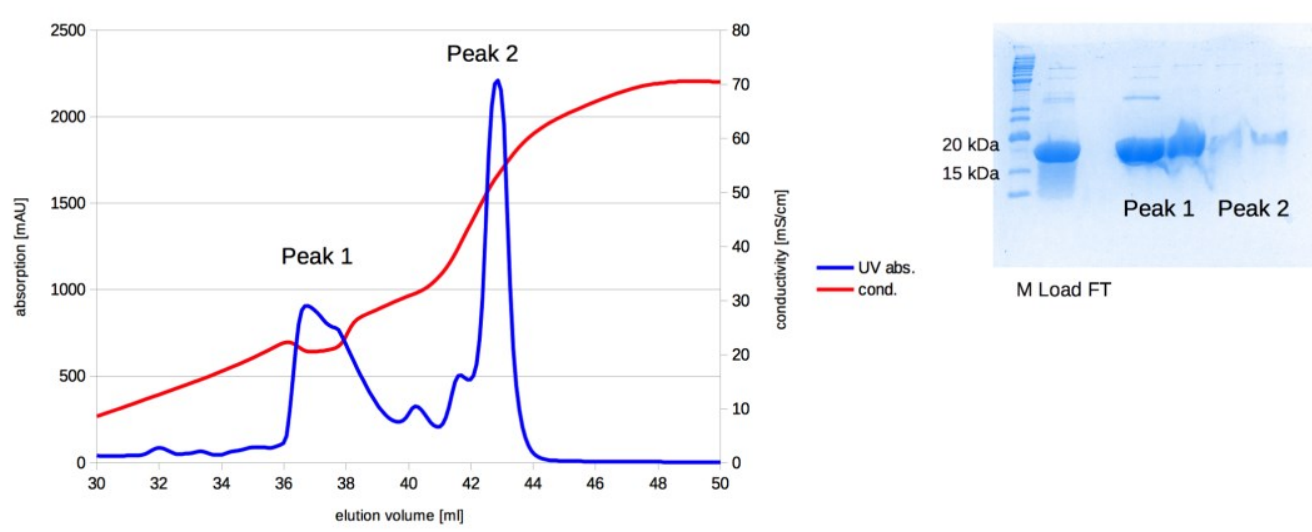

b

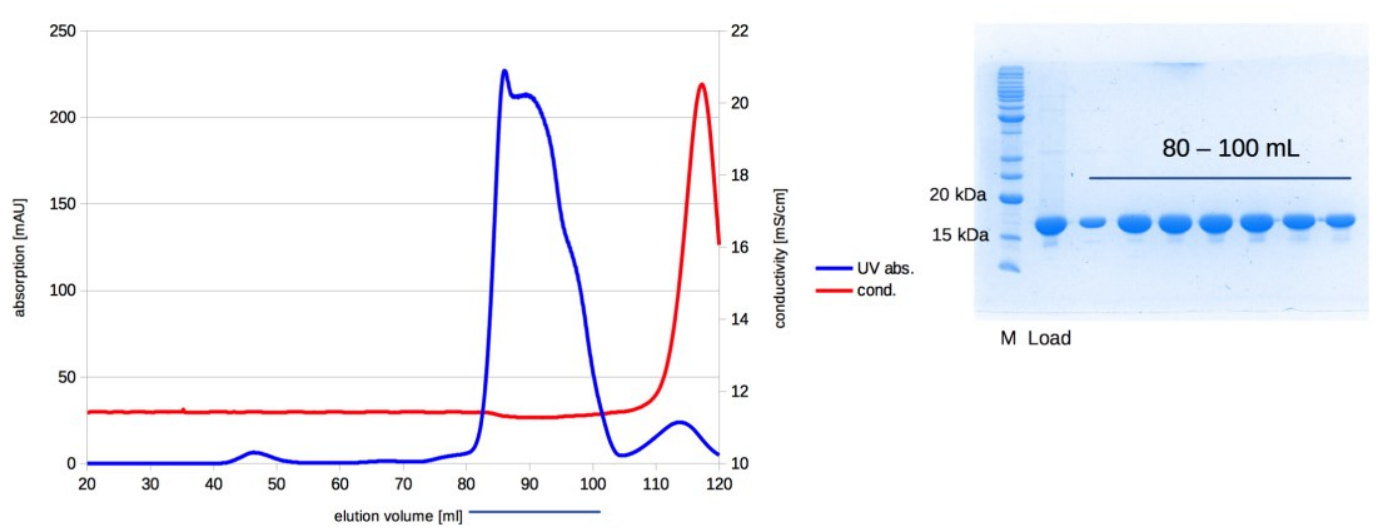

Figure 2-1. Purification of $\mathrm{N}$-terminally acetylated $\alpha$-synuclein.

(a) Mono Q 5/50 anion exchange column run. (b) Gel filtration on Superdex 200 10/300 GL. Using GE Äkta pure, 15\% SDS-PAGE, M: Marker (BenchMark, Invitrogen), FT: flow through.

\subsection{SEC-MALS}

The standard technique multi-angle light scattering ${ }^{141}$ coupled with size exclusion chromatography (SEC-MALS ${ }^{142}$ ) was used for the biophysical characterization of monomeric in vitro aSyn as described before by Burré et al. ${ }^{143}$. The combination of MALS with analytical size exclusion allows absolute mass determination at any position of the elution chromatogram characterizing the different conformational species of soluble protein as well as the analysis of mixed samples.

$200 \mu \mathrm{L}$ of aSyn and non-acetylated $\alpha$-synuclein at a concentration of $50 \mu \mathrm{M}$ were injected onto a Superdex 200 10/300 GL analytical column (GE) using the HPLC system 1260 Infinity with UV detection unit (Agilent Technologies) in combination with the Wyatt system: MALS detector miniDAWM TREOS, RI detector Optilab T-rEX and online QELS detector DynaPro NanoStar. The data analysis was performed with the 
Wyatt Astra software package (Astra 7) using a standard BSA (Sigma) protein solution run at a concentration of $1 \mathrm{mg} / \mathrm{ml}$ for peak alignment and band broadening.

\subsection{Mass spectrometry of aSyn}

Kerstin Overkamp (NMR2, Max-Planck-Chemistry of Biophysical Chemistry, Göttingen, Germany) performed all mass spectrometry experiments. She was provided with a $1 \mathrm{mg} / \mathrm{ml}$ aSyn solution in HEPES buffer. N-terminal acetylation of aSyn was validated using a single quadrupole mass detection system (SQ Detector 2) from Waters in combination with a HPLC system from ACQUITY with diode-array detectors (DAD). The DAD spectrum combines all absorption properties of the sample between 210 and $400 \mathrm{~nm}$. Mass-to-charge ratios (m/z) from 400 to 2500 Da were recorded. For HPLC, a XSelect Peptide CSH C18 column from Waters was chosen with buffers A: $0.1 \%$ TFA in $\mathrm{H}_{2} \mathrm{O}$ (deionized) and $\mathrm{B}$ : $0.1 \%$ TFA in acetonitrile. The theoretical molecular weight $\left(\mathrm{M}_{\mathrm{w}}\right)$ of $\mathrm{N}$-terminally acetylated aSyn is: $14.502 \mathrm{kDa}$ (Appendix: 7.1.2).

\subsection{Patient material}

The patient material used in this study was kindly provided by Glenda M. Halliday (Brain and Mind Centre, Sidney Medical School, Sidney, Australia). Table 3 summarizes the anonymized patient data.

\begin{tabular}{lrlrlr}
\hline $\begin{array}{l}\text { Disease } \\
\#\end{array}$ & Age & Gender & $\begin{array}{l}\text { Postmortem } \\
\text { delay (hrs) }\end{array}$ & Cause of death & $\begin{array}{l}\text { Disease duration } \\
\text { (yrs) }\end{array}$ \\
\hline PD1 & 79 & Male & 17 & Acute myocardial infarction & 7 \\
PD2 & 82 & Male & 22 & Cardiorespiratory failure & 7 \\
PD3 & 81 & Female & 29 & Cardiorespiratory failure & 22 \\
\hline MSA1 & 82 & Male & 8 & Cardiorespiratory failure & 7 \\
MSA2 & 71 & Female & 19 & Hypostatic pneumonia & 6 \\
\hline DLB1 & 69 & Male & 28 & Cardiorespiratory failure & 3 \\
DLB2 & 78 & Male & 3 & Anorexia/cachexia & 7 \\
\hline
\end{tabular}

Table 3. Demographics of donor brains from synucleinopathy patients.

PD: Parkinson's disease, MSA: multiple system atrophy, DLB: Dementia with Lewy bodies. 


\subsection{PMCA and aSyn aggregation}

\subsubsection{Homogenization of patient's material and PMCA}

The human amygdala samples of the synucleinopathy patients (Table 3 ) were homogenized and amplified by PMCA as described earlier by Jung et al. ${ }^{106}$ (Figure 2-2). Homogenization and PMCA was done by Byung Chul Jung (Department of Biomedical Sciences, Neuroscience Research Institute, Seoul, Korea).

The amygdala samples were diluted $10 \%(\mathrm{w} / \mathrm{v})$ in PBS homogenization buffer (1\% Triton X-100 in PBS, Roche protease inhibitor added) and sonicated with a Vibracell sonicator from Sonics. Protein misfolding cyclic amplification (PMCA) was performed with a Qsonica system comprising a microplate horn, a sound enclosure and a thermoelectric chiller. aSyn was diluted in PBS conversion buffer (1\% Triton X100 in PBS, Roche protease inhibitor added) to a final concentration of $2.5 \mu \mathrm{M} .50 \mu \mathrm{l}$ of aSyn were transferred into PCR tubes that contained $2.5 \mu \mathrm{g}$ of brain homogenates of the different patient material. Prior to PMCA, three PTFE beads were added to each PCR tube. The samples were subjected to cycles of $20 \mathrm{sec}$ sonication (amplitude 1\%) and $29 \mathrm{~min} 40 \mathrm{sec}$ of incubation at $37^{\circ} \mathrm{C}$. Total duration of the PMCA was 2 days.



Figure 2-2. Basic principle of cyclic amplification of aSyn fibrils using PMCA.

Taken from Jung et al. ${ }^{106}$.

\subsubsection{Preparation of aSyn fibrils}

aSyn aggregation was performed at $37^{\circ} \mathrm{C}$ using $250 \mu \mathrm{M}$ monomeric aSyn taken from the supernatant (SN) of freshly thawed aSyn on ice after ultracentrifugation (UC) for 1 hour and contained $0.02 \% \mathrm{NaN}_{3}$. All UC steps were performed on an Optima MAX-XP (Beckman Coulter) using a TLA 100.3 rotor with a rotor speed of 55,000 rpm. aSyn concentrations were determined by UV absorption $\left(5960 \mathrm{M}^{-1} \mathrm{~cm}^{-1}\right)$ at $280 \mathrm{~nm}$ using an Eppendorf BioSpectrometer. Fibrillization was monitored by ThT fluorescence (see section 2.12). 


\section{De novo aggregation}

In case of de novo fibrillization of high (hsAsyn; $50 \mathrm{mM}$ Tris- $\mathrm{HCl}, 150 \mathrm{mM} \mathrm{KCl}, \mathrm{pH} 7.5$, $0.02 \% \mathrm{NaN}_{3}$ ) and low salt (IsAsyn; $5 \mathrm{mM}$ Tris- $\mathrm{HCl}, \mathrm{pH} 7.5,0.02 \% \mathrm{NaN}_{3}$ ) in vitro polymorphs, monomeric aSyn (unlabeled and ${ }^{15} \mathrm{~N}$-labeled) was dialyzed against the respective aggregation buffer at $4{ }^{\circ} \mathrm{C}$ o/n, followed by fibrillization as described in Bousset et al. ${ }^{122}$. After harvesting (see fibril harvest) the in vitro fibrils, the fibril pellets were either directly used in hydrogen-deuterium (HD) exchange experiments or for subsequent seeded aggregation experiments the de novo aggregated fibril pellets were resuspended in fresh aggregation buffer to a final concentration of $250 \mu \mathrm{M}$, water bath (Bandelin Sonorex) sonicated for $1 \mathrm{~min}$, aliquoted and shock-frozen in liquid nitrogen for storage at $-80^{\circ} \mathrm{C}$.

\section{$\underline{\text { In vitro seeded aggregation, high and low salt }}$}

Seeded aggregation of the in vitro polymorphs was performed in the same manner as de novo aggregation, but adding $0.5 \%(\mathrm{w} / \mathrm{w})$ of room temperature thawed seeds to the aggregation reaction followed by fibril harvest through UC. If not stated otherwise, all data reported for in vitro polymorphs were obtained through seeding.

\section{Seeded aggregation of synucleinopathic fibrils obtained through PMCA}

For the patient-derived aSyn fibrils, 0.5\% (w/w) PMCA end product was added to 250 $\mu \mathrm{M}$ aSyn stock solution (50 mM HEPES, $100 \mathrm{mM} \mathrm{NaCl}, \mathrm{pH} 7.4,0.02 \% \mathrm{NaN}_{3}$ ) and initially water bath sonicated for 10 minutes. This mixture was aggregated under quiescent conditions in $1.5 \mathrm{ml}$ Eppendorf cups in a Heratherm (ThermoScientific) incubator (Figure 2-3) at $37{ }^{\circ} \mathrm{C}$. After complete fibril formation the fibrils were harvested.

\section{Fibril harvest}

Fibrils were harvested by UC ( $55 \mathrm{krpm}$ ) for 2 hours at $25^{\circ} \mathrm{C}$ and the $\mathrm{SN}$ was separated from the pellet. aSyn concentrations in the $\mathrm{SN}$ were determined spectrophotometrically to estimate fibril yield. 




Figure 2-3. Aggregation scheme for PMCA end products.

\subsection{Negative stain transmission electron microscopy}

Electron microscopy experiments were performed by Gudrun Heim (EM-Facility, MaxPlanck-Institute for Biophysical Chemistry, Göttingen, Germany). Samples were adsorbed onto 400 mesh carbon-coated copper grids and the buffer was removed using a filter paper. Subsequently, samples were stained by the addition of $1 \%$ uranyl acetate solution, which was subsequently dried with a filter paper. The grids were imaged using a FEI CM 120 electron microscope with a Tietz F416 CMOS camera.

\subsection{Thioflavin T fluorescence}

The assembly of aSyn fibrils was monitored over time by Thioflavin T (ThT) fluorescence. ThT dye's application in in vitro binding of amyloid fibrils was described by Naiki et al. ${ }^{144}$. A ThT stock solution of $5 \mathrm{mM}$ ThT in deionized water was produced, sterile filtered, aliquoted and stored at $-20{ }^{\circ} \mathrm{C}$. For each aggregation assay a fresh $10 \mu \mathrm{M}$ ThT glycine buffer solution (50 mM glycine in deionized water, $\mathrm{pH}$ adjusted to 8.8 and sterile filtered) was produced. The ThT solution was always kept in the dark and stored at RT. $2 \mu$ laliquots of aSyn aggregation sample (de novo and/or seeded) were taken at different time points from the total sample volume and added to $200 \mu \mathrm{l}$ of $10 \mu \mathrm{M}$ ThT in glycine buffer to monitor the fibril assembly progress via a Cary Eclipse fluorescence spectrophotometer (Agilent Technologies). The samples were thoroughly mixed at RT and $180 \mu \mathrm{l}$ were transferred to a black 96-well plate (Greiner) and the maximum emission value was determined at $482 \mathrm{~nm}$ exciting the sample well at 440 $\mathrm{nm}$ (ex. bandwith: $10 \mathrm{~nm}$, em. bandwith: $10 \mathrm{~nm}$, gain: 600V PMT detector). 


\subsection{CD spectroscopy}

Circular dichroism (CD) spectroscopy is a powerful tool to estimate the secondary structure elements of proteins in the far-ultraviolet (UV) spectrum ${ }^{145}$.

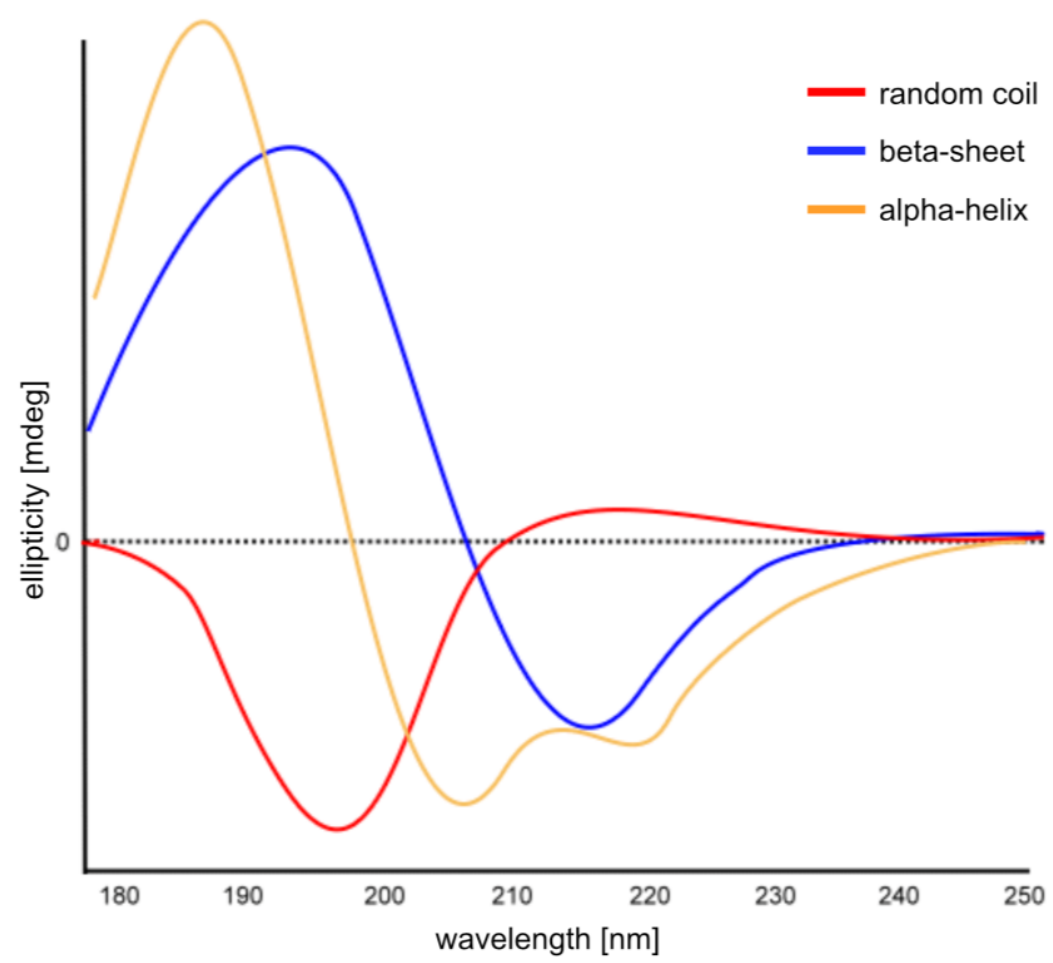

Figure 2-4. Overview of CD spectra of pure secondary structures.

Adapted from Farid, R. S. ${ }^{146}$

For characterization of aSyn fibrils, $5 \mu \mathrm{L}$ of aggregation sample were centrifuged for 10 minutes at 20,000 $\mathrm{g}$ in an Eppendorf centrifuge 5424. The pellets were resuspended in $60 \mu \mathrm{l}$ deionized water after discarding the supernatants and transferred to a $0.02 \mathrm{~cm}$ pathlength cuvette. CD data were collected from $185 \mathrm{~nm}$ to $260 \mathrm{~nm}$ by using a Chirascan qCD spectrometer (Applied Photophysics) at $20^{\circ} \mathrm{C}, 1.5$ time-per-point (s) in $1 \mathrm{~nm}$ steps. The datasets were averaged from two repeats. All spectra were baseline corrected against deionized water and smoothed (window size: $8,4^{\text {th }}$ order polynom).

\subsection{Fluorescent dye binding}

The dyes (E,E)-1-fluoro-2,5-bis(3-hydroxycarbonyl-4-hydroxy)styrylbenzene (FSB) and curcumin were purchased from Santa Cruz Biotechnology and Sigma-Aldrich. The polythiophene acetic acid derivative HS-68 was synthesized by the Synthetic Chemistry Facility (Max-Planck-Institute for Biophysical Chemistry) according to published procedures ${ }^{147}$ as a sodium salt, see (Appendix: Figure 7-1). 




Figure 2-5. Chemical structural formula of fluorescent dyes.

The fluorescent dyes curcumin and FSB were dissolved in DMSO, HS-68 in deionized water at a stock solution of $5 \mathrm{mM}$. The stock solution was then aliquoted and stored at $-20{ }^{\circ} \mathrm{C}$ in the dark. Working solutions were freshly prepared from the dye stocks to a final concentration of $50 \mu \mathrm{M}$ of the respective dye in $50 \mathrm{mM}$ HEPES, $100 \mathrm{mM} \mathrm{NaCl}$, $\mathrm{pH} 7.4$, the final DMSO concentration for curcumin and FSB was $1 \%$.

$100 \mu \mathrm{L}$ of working dye solution was mixed thoroughly with $10 \mu \mathrm{L}$ of aSyn fibrils to a final protein-to-dye ratio of $\sim 1: 6$. The samples were transferred to a 96-well microplate (PS, $\mu C L E A R$, black, chimney well from Greiner). The fluorescence measurements were performed on a Spark 20M reader (TECAN) and read from the top at $25{ }^{\circ} \mathrm{C}$ with z-Position: $17191 \mu \mathrm{m}$, intensity 505 averaged over 30 flashes, $2 \mathrm{~nm}$ steps.

Curcumin: $\quad$ ex.: $440 \mathrm{~nm}$, gain: 80 , ex. bandwidth: $20 \mathrm{~nm}$, em. bandwidth: $20 \mathrm{~nm}, 485-800 \mathrm{~nm}$. FSB: $\quad$ ex.: $380 \mathrm{~nm}$, gain: 60 , ex. bandwidth: $10 \mathrm{~nm}$, em. bandwidth: $10 \mathrm{~nm}, 450-800 \mathrm{~nm}$. HS-68: $\quad$ ex.: $450 \mathrm{~nm}$, gain: 60 , ex. bandwidth: $10 \mathrm{~nm}$, em. bandwidth: $10 \mathrm{~nm}, 510-800 \mathrm{~nm}$.

All spectra were baseline subtracted by dye buffer blank measurements and smoothed (windows size: $8,4^{\text {th }}$ order polynom). 


\subsection{NMR spectroscopy}

2.15.1 Heteronuclear single quantum coherence (HSQC)

Due to the small gyromagnetic ratio of hetero nuclei (such as ${ }^{15} \mathrm{~N}$ and ${ }^{13} \mathrm{C}$ ) in comparison to protons $\left({ }^{1} \mathrm{H}\right)$, a vast amount of complex inverse magnetization transfer based pulse sequences were developed for liquid-state NMR spectroscopy achieving magnetization transfer from proton to the hetero nuclei of choice, followed by variable evolution periods under defined conditions and eventually ending with back-transfer of magnetization to ${ }^{1} \mathrm{H}$ for detection.

a

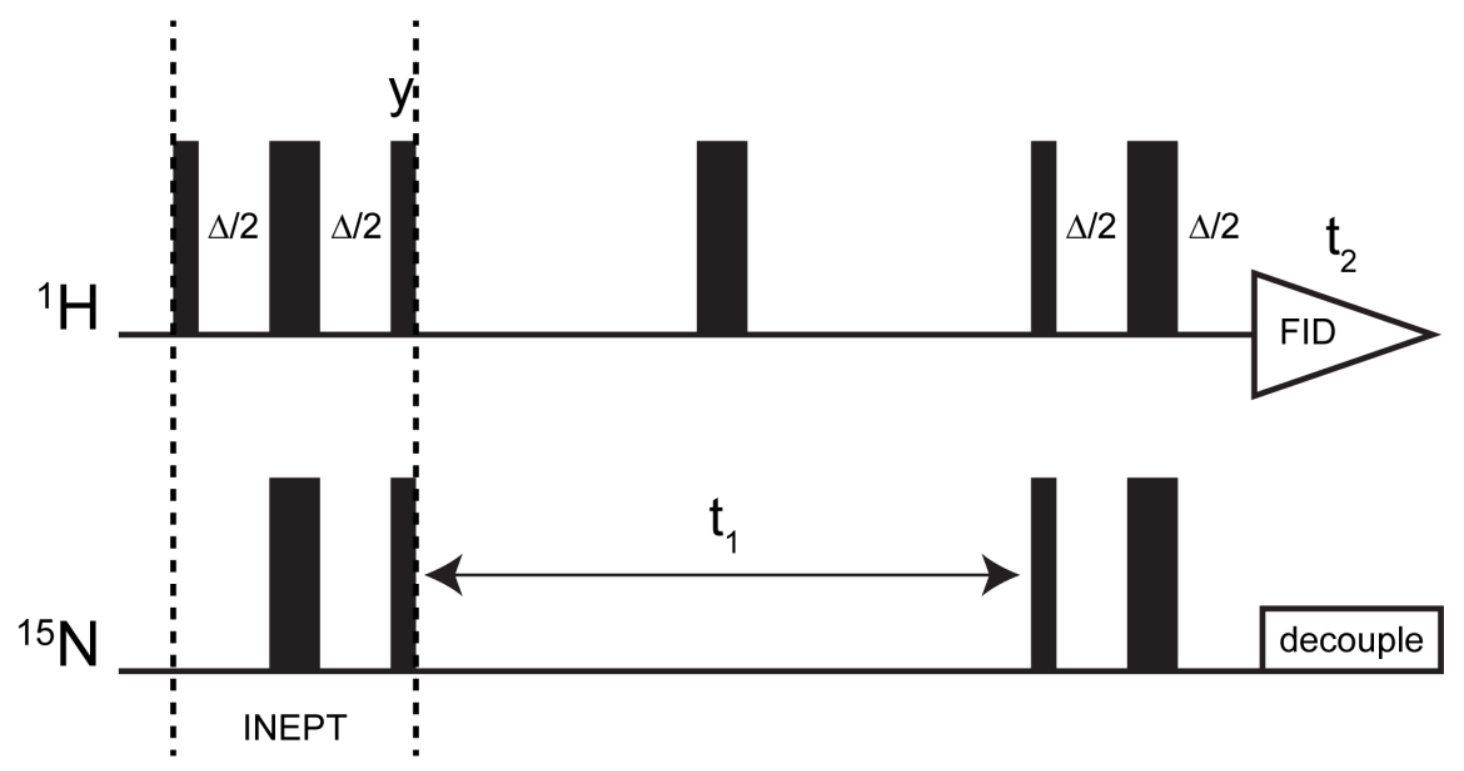

b



Figure 2-6. Simplified scheme of 2D HSQC NMR spectroscopy.

(a) Schematic visualization of a $\mathrm{HSQC}$ pulse sequence. Narrow bars represent $90^{\circ}$, wide bars $180^{\circ}$ pulses. All pulses have phase $\mathrm{x}$ if not stated otherwise. The INEPT element is indicated by dashed lines. $\Delta$ is set to $1 /\left(2 J_{\mathrm{NH}}\right)$ for complete transformation of in-phase into anti-phase 
magnetization. The block "decouple" stands for the ${ }^{15} \mathrm{~N}$ broad band decoupling pulse during acquisition. (b) The HSQC pulse sequence achieves magnetization transfer from the amide attached proton $\left({ }^{1} \mathrm{H}\right)$ to the nitrogen $\left({ }^{15} \mathrm{~N}\right)$ of the amide bond shown for the amino acid backbone of a polypeptide sequence. INEPT: insensitive nuclei enhancement through polarization transfer.

In case of the ${ }^{1} \mathrm{H}-{ }^{15} \mathrm{~N}$ HSQC pulse sequence ${ }^{148}$ (Figure 2-6 a) magnetization transfer from the proton to the ${ }^{15} \mathrm{~N}$ hetero nucleus is achieved via the INEPT element, evolution of chemical shift of ${ }^{15} \mathrm{~N}$ occurs then during $\mathrm{t}_{1}$ but not for ${ }^{1} \mathrm{H}$, a $180^{\circ} \times$ pulse centered in $\mathrm{t}_{1}$ applied only on ${ }^{1} \mathrm{H}$ refocuses the offset of ${ }^{1} \mathrm{H}$. The evolution period $\mathrm{t}_{1}$ is followed by a reverse-INEPT block restoring in-phase magnetization on ${ }^{1} \mathrm{H}$ preceding acquisition under $\mathrm{t}_{2}$ with broad band decoupling on ${ }^{15} \mathrm{~N}$ (Figure 2-6 a).

Recording a set of one-dimensional proton FIDs during $t_{2}$ incrementing the evolution period $t_{1}$ results in a two-dimensional dataset in the time domains $t_{2}$ and $t_{1}$. Operations can be directly performed on the time domains such as baseline correction, linear prediction and window multiplication. Eventually using the fast Fourier transform algorithm, the dataset is converted into a $2 \mathrm{D}{ }^{1} \mathrm{H}-{ }^{15} \mathrm{~N} \mathrm{HSQC}$ spectrum in the frequency domain plotted in parts per million (ppm). The final HSQC spectrum is obtained using empirically optimized phase correction. Since the HSQC pulse program correlates the protons directly bonded to nitrogen $\left({ }^{15} \mathrm{~N}\right)$, it is a powerful tool for protein science. The amide backbone of proteins and polypeptides own for each amino acid residue one proton single-bonded to nitrogen (Figure 2-6 b), except for proline residues (Pro, P) that cannot be studied by HSQC spectra.

The HSQC pulse sequence ${ }^{149-151}$ used in this study is shown in Appendix 7.5 in detail and is more complex than the scheme shown in Figure 2-6 a, however the same basic principles hold true. 


\subsubsection{Resonance assignment}

Backbone resonance assignments of aSyn in the dissolving buffer (4M GdSCN, 0.4\% formic acid, $\mathrm{pH} 2.5$ ) at a protein concentration of $600 \mu \mathrm{M}$ were obtained with the help of 3D $\mathrm{HNCO}^{152,153,154}, \mathrm{HN}(\mathrm{CA}) \mathrm{CO}^{155,154}, \mathrm{HNCA}^{152,153,154}, \mathrm{HN}(\mathrm{CO}) \mathrm{CA}^{153,154}$ and $\mathrm{HNCACB}^{156,157}$ experiments ${ }^{158}$, in which indirect frequency dimensions were sampled in a non-uniform manner (NUS) ${ }^{159}$ for the experiments stated in Table 4 using standard Bruker pulse sequences. The spectra were processed using nmrPipe ${ }^{136}$ with SMILE ${ }^{160}$ for NUS data and analyzed with CcpNmr Analysis software package ${ }^{139}$.

\begin{tabular}{llll}
\hline Experiment & Parameters & Spectrometer & Pulse sequence \\
\hline HNCO & $110 \times 60 \times 2048$, ns: 16 & $600 \mathrm{MHz}$ & hncogpwg3d \\
HNCO & $128 \times 80 \times 2048$, ns: 16, NUS $(25 \%)$ & $800 \mathrm{MHz}$, cryoprobe & hncogpwg3d_sct \\
HN(CA)CO & $105 \times 60 \times 2048$, ns: 32 & $600 \mathrm{MHz}$ & hncacogpwg3d \\
HNCA & $115 \times 60 \times 2048$, ns: 32, NUS $(25 \%)$ & $800 \mathrm{MHz}$, cryoprobe & hncagpwg3d_sct \\
HN(CO)CA & $104 \times 60 \times 2048$, ns: 24 & $600 \mathrm{MHz}$ & hncocagpwg3d \\
HNCACB & $124 \times 100 \times 2048$, ns: 40, NUS (30\%) & $800 \mathrm{MHz}$, cryoprobe & hncacbgpwg3d_sct \\
\hline
\end{tabular}

Table 4. 3D experiments recorded for resonance assignment of aSyn in dissociation buffer.

\subsubsection{Weighted chemical shift perturbations}

The weighted chemical shifts (CSP) were calculated using the relative chemical shifts in ${ }^{1} \mathrm{H}\left(\delta_{\mathrm{H}}\right)$ and ${ }^{15} \mathrm{~N}\left(\delta_{\mathrm{N}}\right)$ according to:

$$
d=\sqrt{\frac{1}{2} \cdot\left[\delta_{H}^{2}+\left(\frac{1}{5} \cdot \delta_{N}^{2}\right)\right]}
$$

\subsubsection{Secondary chemical shifts}

Secondary chemical shifts were calculated using CcpNmr Analysis software package ${ }^{139}$ based on the assignment (Appendix: Table 5) according to Schwarzinger et al. ${ }^{161}$.

\subsection{Hydrogen-deuterium exchange}

The combination of hydrogen-deuterium (HD) exchange studies with methods of rapid fibril dissociation and liquid-state NMR spectroscopy allows the determination of deuterium incorporation levels with single-residue resolution ${ }^{162,163,164}$. To further enhance the distinction for residues with different amide proton exchange rates, a mixture of $\mathrm{H}_{2} \mathrm{O}$ and $\mathrm{D}_{2} \mathrm{O}$ in the dissociation buffer was used. Hydrogen-deuterium (HD) 
exchange coupled to nuclear magnetic resonance (NMR) spectroscopy (Figure 2-7) was performed according to a previously established protocol by Cho et. al. ${ }^{162}$.

\subsubsection{Forward exchange}

Previous HD exchange studies conducted on in vitro aggregated aSyn fibrils established that a forward ( $\mathrm{fw}$ ) exchange time of 1.5 days allows robust determination of residue-specific protonation level ${ }^{162,164}$. To slow down HD exchange and thus allow a better definition of regions with small differences in solvent protection, forward HD exchange was done in $0.1 \%$ formic acid ( $p D 4.0$ ) in $99.9 \% \mathrm{D}_{2} \mathrm{O}$ at $37^{\circ} \mathrm{C}$. The forward $\mathrm{HD}$ exchange of amide protons in aSyn fibrils was stopped after 1.5 days, fibrils were collected by ultracentrifugation ( 2 hours, 200,000 g), frozen in liquid nitrogen and stored in a refrigerator at $-80^{\circ} \mathrm{C}$.

\subsubsection{Back-exchange and NMR real-time acquisition}

Subsequently, back-exchange in the dissociation buffer (4 M guanidine thiocyanate (GdSCN), 0.4\% formic acid, $\mathrm{pH} 2.5$, in $60 \% \mathrm{D}_{2} \mathrm{O}$ ) was followed by $120-1402 \mathrm{D}{ }^{1} \mathrm{H}-{ }^{15} \mathrm{~N}$ heteronuclear single quantum coherence (HSQC) experiments (experimental time for each HSQC: 8 min $16 \mathrm{sec}$; number of increments in ${ }^{15} \mathrm{~N}$-dimension: 128) recorded over a time period of $\sim 19$ hours at a temperature of $3{ }^{\circ} \mathrm{C}$. NMR experiments were measured on a Bruker $800 \mathrm{MHz}$ spectrometer equipped with a $5 \mathrm{~mm}$ triple-resonance, pulsedfield z-gradient cryoprobe. Preparative steps such as shimming and temperature equilibration resulted in a dead time of about 7 minutes (measured for each data set) before the start of the first HSQC.

\subsubsection{Data analysis}

Acquired spectra were processed with linear prediction for the ${ }^{15} \mathrm{~N}$ dimension and analyzed by NMRPipe ${ }^{136}$. The relative intensity (height) data for 103 HSQC-separated aSyn residues were extracted from $120-1402 \mathrm{D}{ }^{1} \mathrm{H}-{ }^{15} \mathrm{~N}$ HSQC spectra using the centered peak position of the final fully exchanged HSQC spectrum of the series normalized to a value of 1.0 using NMRPipe ${ }^{136}$. For each aSyn residue the backexchange curves were fitted to a single exponential decay using Prism (Graphpad). Knowing the exact dead time and HSQC experiment duration the relative $t_{0}$ intensity level was back-calculated and the scale was transformed into absolute protonation levels by using the average maximum intensity value of the five residues with highest signal intensity. 

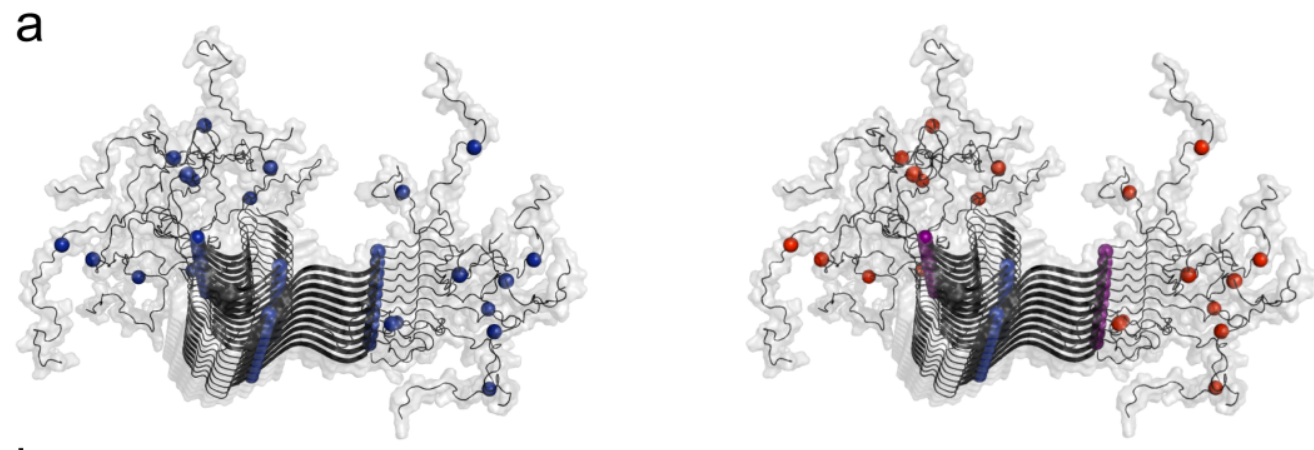

b
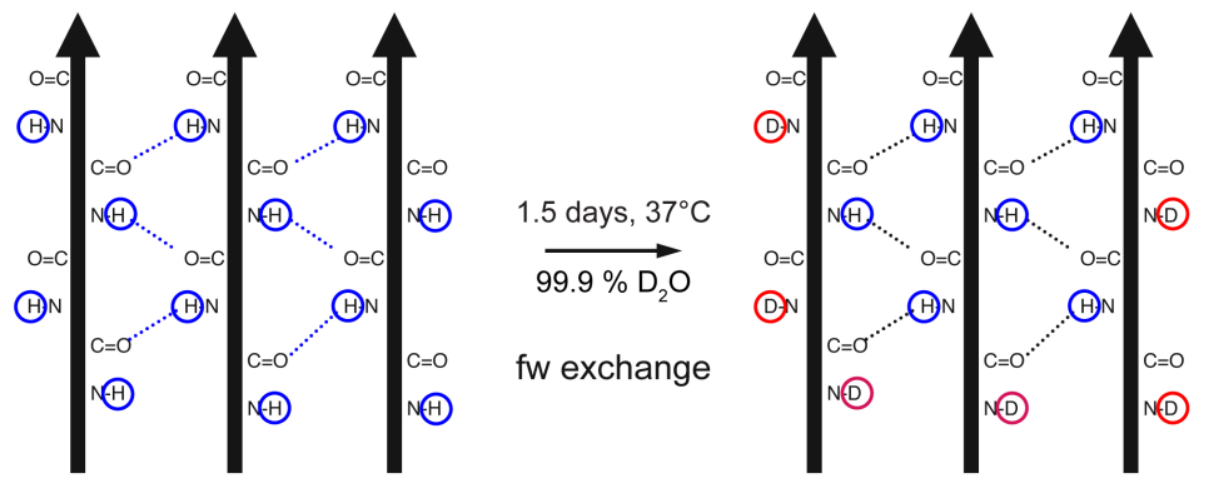

Harvested aSyn fibrils fully protonated $\mathrm{NH}$-backbone

Mixed protonation level of $\mathrm{NH}$-backbone after fw exchange
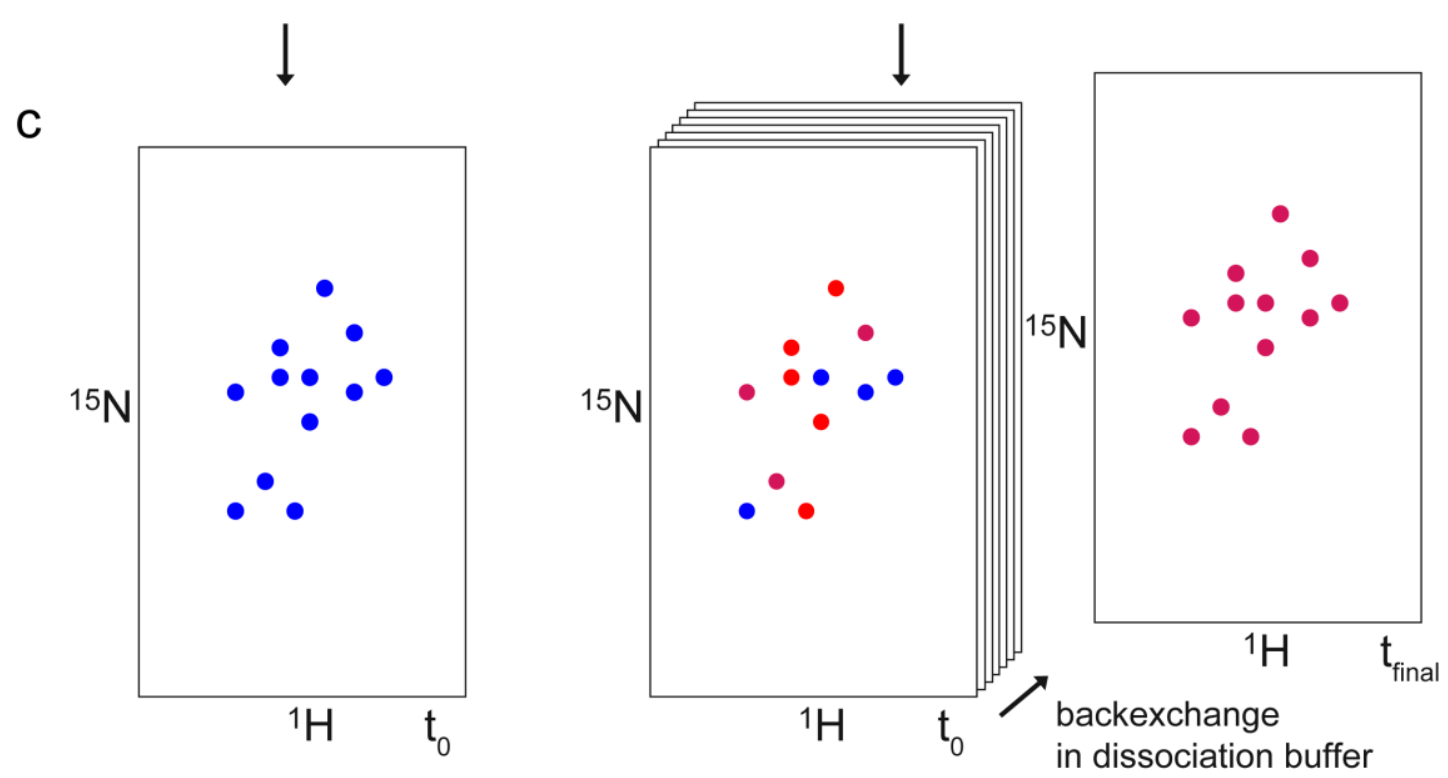

Figure 2-7. Schematic description of HD exchange coupled to NMR spectroscopy.

(a) aSyn fibril model before (left) and after fw exchange showing selected protons of the $\mathrm{NH}$ backbone, blue: fully protonated, red: fully deuterated, purple: intermediate protonation level. (b) Simplified HD fw exchange scheme. (c) HSQC intensities coding for protonation level. 


\subsection{EPR spectroscopy}

Monomeric aSyn was aggregated under quiescent conditions after initial 10 minutes water bath sonication as described in Chapter 2.10 .2 using $0.5 \%(\mathrm{w} / \mathrm{w})$ of aSyn fibrils (hsAsyn, IsAsyn, PD, MSA and DLB) as seeds in the presence of 1:30 MTSL-labeled aSyn-T54C/A90C following an established protocol of Pornsuwan S. et al. ${ }^{165}$. The total aSyn concentration was $200 \mu \mathrm{M}, 193.3 \mu \mathrm{M}$ of aSyn and 6.7 $\mu \mathrm{M}$ of MTSL-labeled aSynT54C/90C. MTSL-labeled aSyn, see Figure 2-8, was provided by Stefan Becker and Karin Giller (NMR2, Max-Planck-Institute for Biophysical Chemistry, Göttingen, Germany). The mutant protein aSyn-T54C/A90C was constructed using the QuikChange site-directed mutagenesis kit (Stratagene), and the introduced modifications were verified by DNA sequencing. Protein expression and purification was performed as described ${ }^{38}$. The reaction of the aSyn-T54C/A90C mutant protein with the nitroxide spin label MTSL was carried out as described ${ }^{30}$.

In brief, using size exclusion chromatography (PD-10 columns, Amersham Pharmacia Biosciences) the reducing agent DTT was removed from the buffer before labeling procedure. The $\alpha$-synuclein mutant (aSyn-T54C/A90C) was then equilibrated in Tris-HCl buffer (20 mM Tris- $\mathrm{HCl}, 100 \mathrm{mM} \mathrm{NaCl}, \mathrm{pH} 7.5)$ in presence of 5-fold molar excess of MTSL. The reaction was performed at $4{ }^{\circ} \mathrm{C}$ and stopped after 16 hours by removal of unreacted MTSL spin label using again PD-10 columns. The spin-labeled aSyn-T54C/A90C was concentrated using Microcon YM-3 (MWCO: 3 kDa) centrifugal filters (Amicon).

After fibril formation the fibrils were pelleted by UC (Optima MAX-XP, TLA 100.3 rotor, $55,000 \mathrm{rpm}, 25{ }^{\circ} \mathrm{C}, 2$ hours) and additionally washed with a fullydeuterated $50 \mathrm{mM}$ HEPES, $100 \mathrm{mM} \mathrm{NaCl}$, pD 7.81 buffer following a final harvest step of 1 hour. The pellet was shock-frozen in liquid nitrogen and stored at $-80^{\circ} \mathrm{C}$. For EPR measurements the respective fibril pellets were thawed at RT and resuspended in $30 \mu$ final volume $\mathrm{D}_{2} \mathrm{O}$ supplemented with $10 \%$ deuterated glycerol- $\mathrm{d}_{8}$ and $15 \mu \mathrm{L}$ were loaded into quartz tubes with an outer diameter of $1.6 \mathrm{~mm}$ and an inner diameter of $1.1 \mathrm{~mm}$. 
a



b

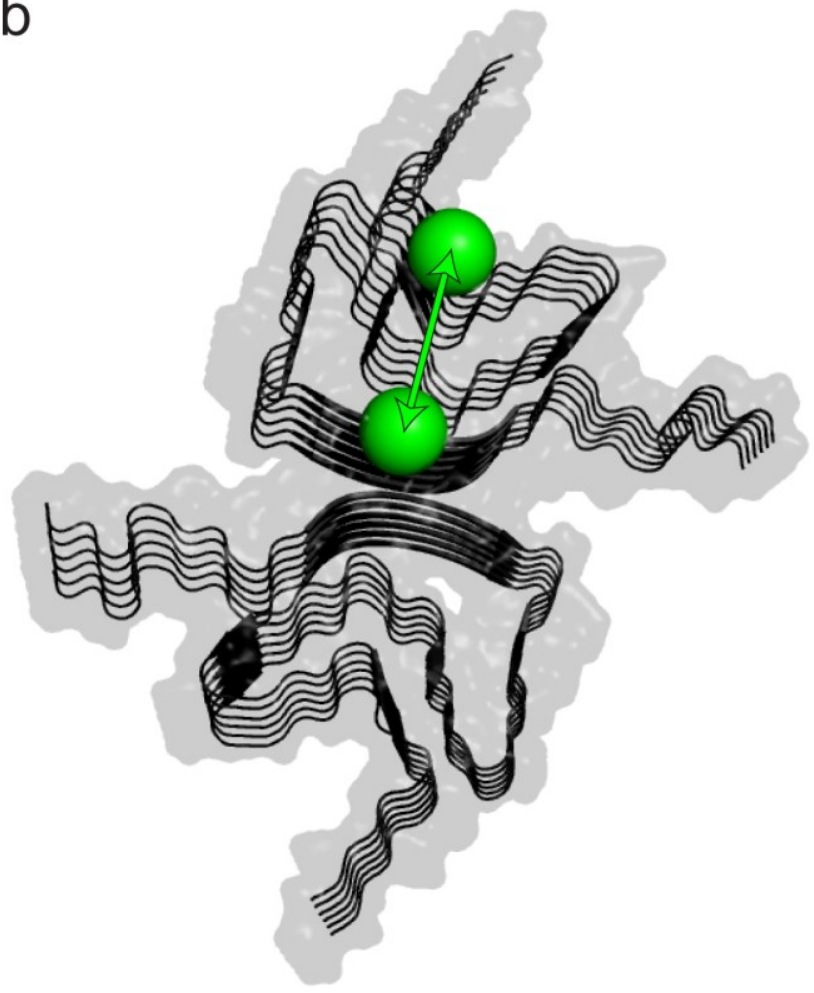

Figure 2-8. MTSL-labeled aSyn A90C/T54C incorporated into fibril structure at 1:30.

(a) MTSL-label attached to cysteine side chain of T54C and A90C mutations of aSyn. (b) Distance determination by EPR indicated with arrow between the two label positions in the structure of aSyn fibrils (PDB: 6A6B).

EPR measurements were done by Shu-Hao Liou (EPR spectroscopy, Max-PlanckInstitute for Biophysical Chemistry, Göttingen, Germany). Room-temperature continuous wave EPR spectra were recorded on an EleXsys E500 spectrometer at Xband (9.8 GHz), equipped with a Super-High $Q$ resonator (ER4122SHQE). Experimental conditions: microwave (MW) power $2 \mathrm{~mW}$, modulation amplitude $5 \mathrm{G}$, conversion time $10 \mathrm{~ms}$ and 150 scans. Continuous wave EPR spectra were simulated using the program 'Chili' in Easyspin software (version 5.1.12) ${ }^{166}$. Two nitroxide species with different weights were used in the simulation. After initial estimation of weights, multiple least-square optimizations were performed, in order to obtain the correlation times and weights for different nitroxide dynamics. For the fitting procedure, hyperfine and $g$ values as well as peak-to-peak line widths were kept within a range consistent with previous reports ${ }^{167}$.

Q-band (34 GHz) PELDOR measurements were performed on a Bruker EleXsys E580 spectrometer, equipped with a pulsed $170 \mathrm{~W}$ Q-band TWT-amplifier (Model 187Ka, Applied Systems Engineering Inc.) and an EN5107D2 EPR/ENDOR probe-head. PELDOR traces were measured using a four-pulse sequence $\left(\frac{\pi^{1}}{2}-\tau_{1}-\right.$ 
$\pi^{1}-\left(\tau_{1}+T\right)-\pi^{2}-\left(\tau_{2}-T\right)-\pi^{1}-\tau_{2}-$ [echo]) with probe pulse $\left({ }^{1}\right)$ and pump pulse $\left({ }^{2}\right)^{168} . T$ was incremented in $8 \mathrm{~ns}$ steps. The pump pulse frequency was located the maximum nitroxide absorption, determined by field-swept echo experiments. The probe pulse frequency was set to $80 \mathrm{MHz}$ lower than the pump frequency $(\Delta v=80$ $\mathrm{MHz}$ ). The probe and pump pulse lengths were determined by a nutation experiment. The length of the probe $\pi$ pulse was 26-32 ns, depending on the $\mathrm{Q}$-value of the cavity. The $\frac{\pi}{2}$ pulse length was set to be identical to the length of $\pi$ pulse by varying the pulse strength or amplitude $\left(w_{1}\right)$. This procedure minimizes the overlap between the pump and probe pulse and thus attenuates the so-called ' $2+1$ ' artifact. The $\pi$ pulse at pump frequency was around 22-26 ns, and $\tau_{1}$ was set to $200 \mathrm{~ns}$, in order to suppress deuterium modulations. DeerAnalysis $2018^{137,138}$ was used to analyze PELDOR spectra. Background subtraction was performed using $2^{\text {nd }}$ polynomial functions, followed by least-square fitting and Tikhonov regularization with L-curve selection. 


\section{Results}

\subsection{Biophysical characterization of monomeric aSyn}

$\mathrm{N}$-terminal acetylation is a post-translational modification commonly occurring in mammalian proteins ${ }^{169}$. $\alpha$-synuclein isolated from humans is considered to be ubiquitously acetylated in its first methionine residue ${ }^{60,170}$. On top of that $\mathrm{N}$-terminally acetylated $\alpha$-synuclein (aSyn) has distinct biophysical properties shown for membrane binding ${ }^{171}$ and protein-metal ion interactions ${ }^{172}$. In addition, aSyn is well known to show high variability in its aggregation behavior and the critical parameter besides working with protein of high purity is the absence of oligomeric higher molecular weight species that promote aggregation and eventually lead to inconsistent aggregation behavior of aSyn in vitro. Therefore, purified recombinant aSyn protein was studied with different biophysical techniques (SEC-MALS, mass spectrometry and NMR spectroscopy) to investigate protein purity, its conformational state as well as the completeness of the $\mathrm{N}$-terminal acetylation introduced as described in section 2.6 (Protein preparation).

By protein purification, purities higher 95\% were consistently obtained. The elution volume of aSyn from a size-exclusion column indicated an intrinsically disordered behavior.

\subsubsection{SEC-MALS}

Analytical size-exclusion coupled to multi-angle light scattering (SEC-MALS) showed that the recombinantly expressed aSyn elutes as a monomer from the analytical size exclusion column (Superdex 200 10/300 GL) under native-like aqueous conditions in solution. The detected Z-average molar mass $\left(\mathrm{M}_{\mathrm{z}}\right)$ for aSyn was $14.1 \pm 1.38 \mathrm{kDa}$ using the $\mathrm{RI}$ detector for concentration determination and three light scattering detectors $\left(45^{\circ}, 90^{\circ}\right.$ and $135^{\circ}$ angle), see Figure $3-1$. The theoretical expected molecular weight for aSyn is $14.50 \mathrm{kDa}$. $\alpha$-synuclein without $\mathrm{N}$-terminal acetylation from a different protein preparation was analyzed for comparison; the detected molecular weight was $13.8 \pm 1.37 \mathrm{kDa}$. The apparent molar mass of aSyn from SEC-MALS was $300 \mathrm{Da}$ higher than for non-acetylated $\alpha$-synuclein and aSyn also eluted slightly earlier from the column indicating a higher molecular weight. For cross-validation the UV signal was used as method for concentration determination showing also higher molecular mass for aSyn at a slightly lower absolute level (data not shown). 
a

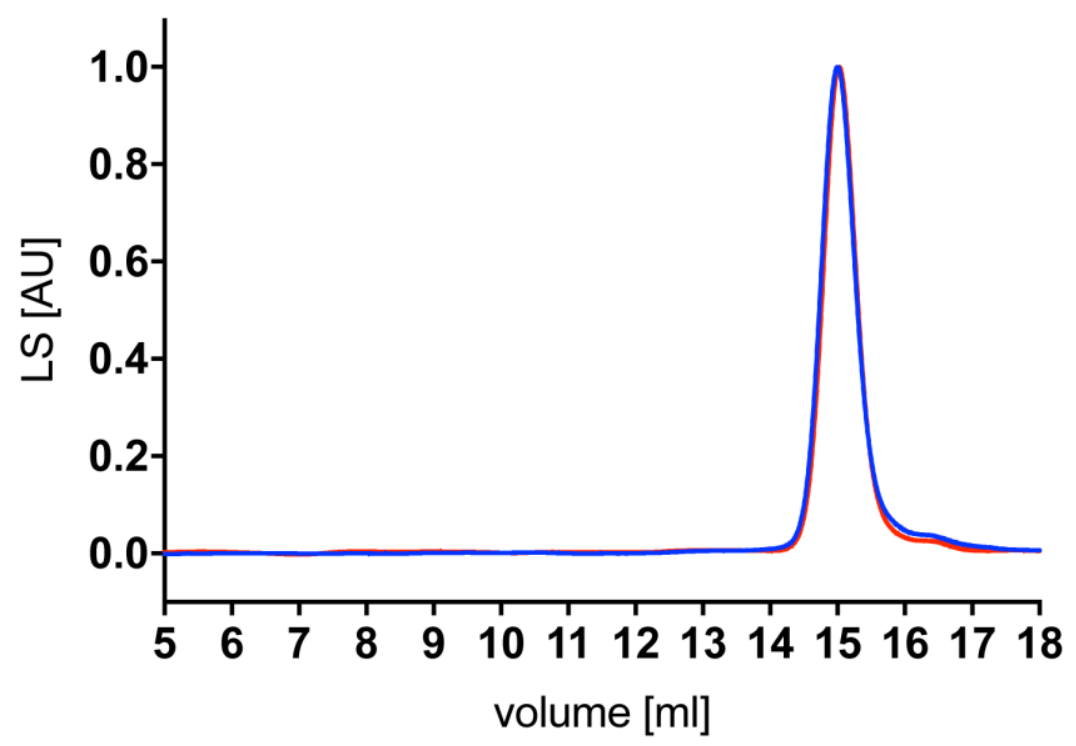

b

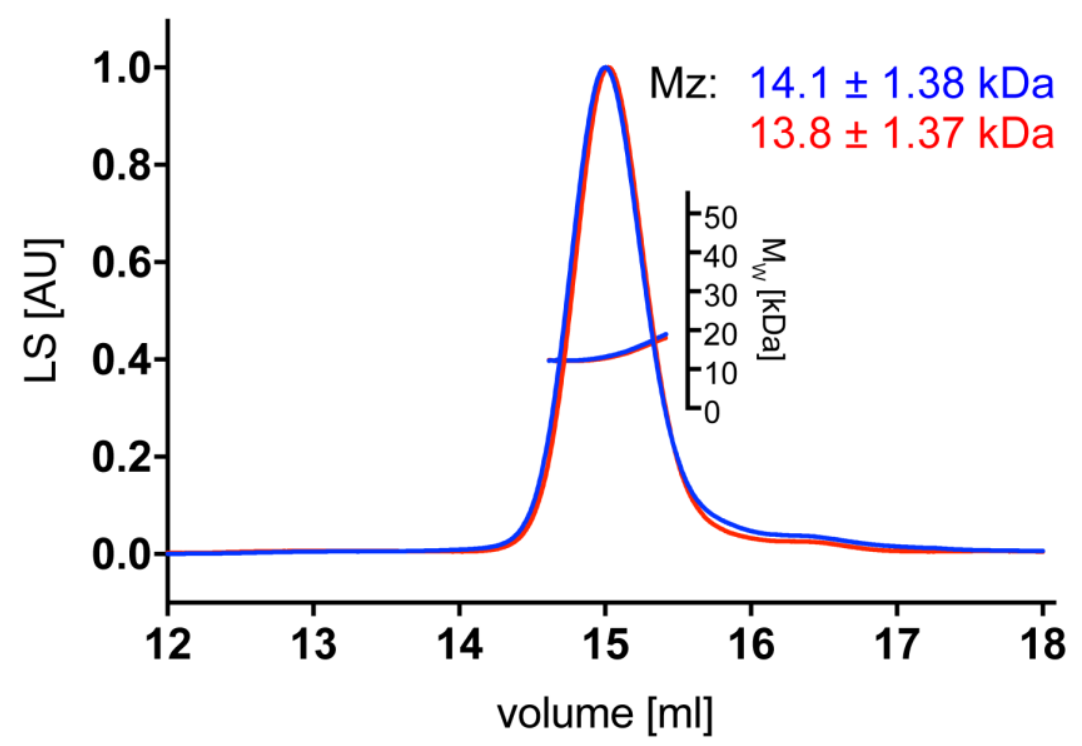

Figure 3-1. SEC-MALS of aSyn in comparison to non-acetylated $\alpha$-synuclein.

(a) SEC-MALS run showing normalized light scattering signal of the $90^{\circ}$ detector. (b) Zoom into the $\alpha$-synuclein elution peak, $\mathrm{M}_{z}$ molecular weight determined by RI signal. Blue: aSyn, red: non-acetylated $\alpha$-synuclein.

\subsubsection{Mass spectrometry}

To further validate the purity of the protein preparation as well as the completeness of the introduced N-terminal acetylation, HPLC in combination with mass spectrometry (MS-ESI) was chosen. A single peak eluted from the column with a retention time of $8.74 \mathrm{~min}$, the detected molecular mass was $14.505 \mathrm{kDa}$. 
a


Figure 3-2 Validation of $\mathrm{N}$-terminal acetylation of aSyn by mass spectrometry.

(a) HPLC run of aSyn. (b) m/z spectrum of aSyn. (c) Calculated average mass for HPLC elution.

\subsubsection{N-terminal acetylation by NMR}

Since the post-translational modification (PTM) of N-terminal acetylation changes the chemical environment of the initial aSyn residues and introduces structural perturbations in the ensemble due to higher rigidity of the $\mathrm{N}$-terminus, two-dimensional heteronuclear correlation NMR experiments $\left({ }^{1} \mathrm{H}-{ }^{15} \mathrm{~N}\right.$ HSQC) are sensitive to it and allow validation of $\mathrm{N}$-terminal acetylation (Figure 3-3). 

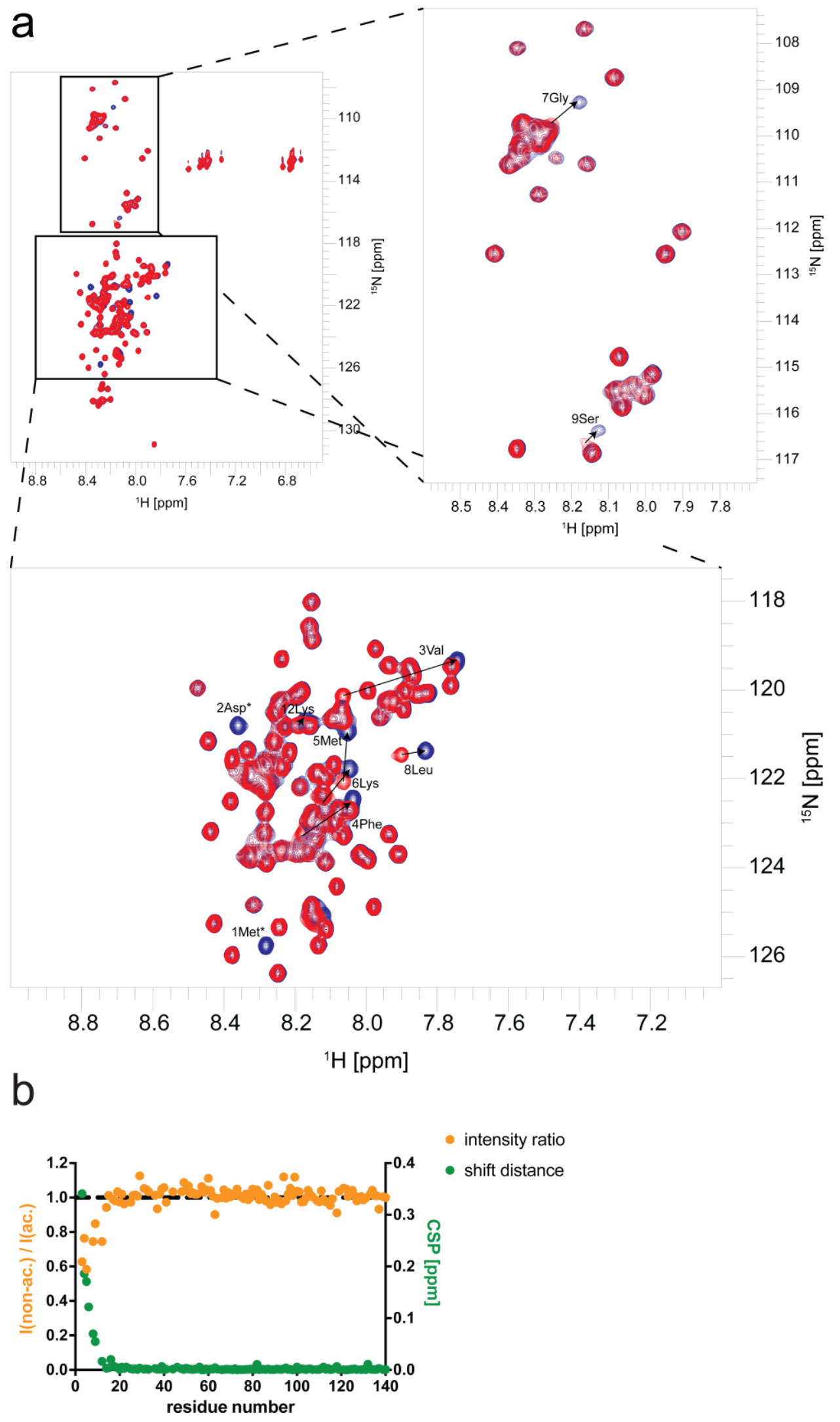

Figure 3-3. N-terminal acetylation validated by NMR.

(a) ${ }^{1} \mathrm{H},{ }^{15} \mathrm{~N}-\mathrm{HSQC}$ spectra at $288 \mathrm{~K}$ in HEPES buffer (50 mM HEPES, $100 \mathrm{mM} \mathrm{NaCl}, \mathrm{pH} 7.4$, $0.01 \% \mathrm{NaN}_{3}$ ). Perturbed residues are indicated in two regions. aSyn in blue, non-acetylated $\alpha-$ synuclein in red. (b) Intensity ratio in orange, chemical shift perturbation in green. 


\subsection{Amplification of aSyn aggregates}

\subsubsection{Amplification of aSyn aggregates from brain extracts}

aSyn aggregates from brain extracts of seven patients diagnosed with either PD, MSA or DLB (Patient material: Table 3) were amplified using protein misfolding cyclic amplification (PMCA) at low concentration of $2.5 \mu \mathrm{M}$ to prevent de novo aggregation. The PMCA end products were then used to seed aggregation under quiescent condition in the presence of unlabeled and ${ }^{15} \mathrm{~N}$-labeled aSyn at $250 \mu \mathrm{M}$. The seeded amplification step was introduced to obtain sufficient quantities of PMCA-amplified amyloid fibrils for further structural analysis.

a

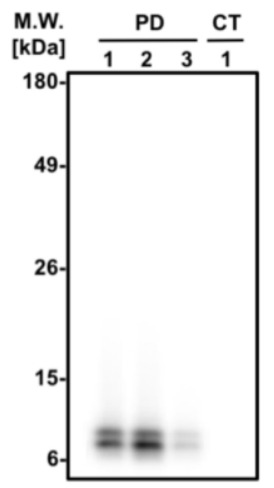

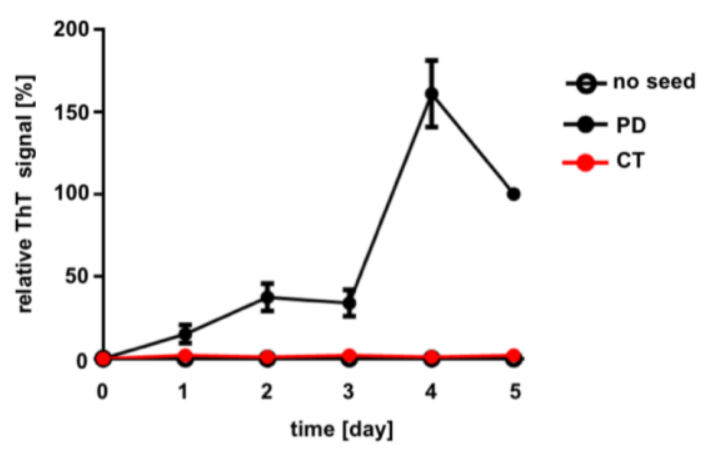

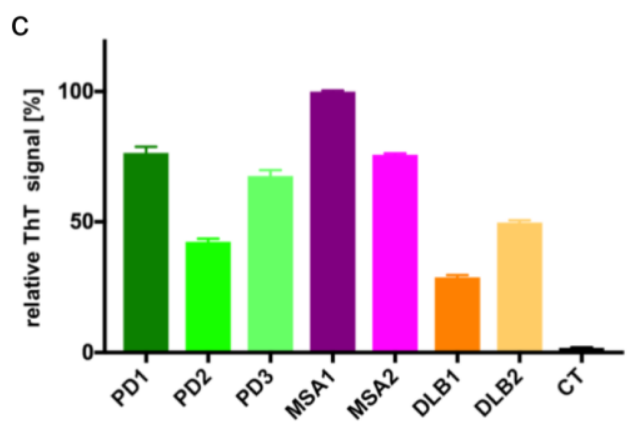

Figure 3-4. Amplification of aSyn from brain extracts.

(a) Immunoblotting of PMCA-amplified aggregates with PK digestion. Similar PK-resistant signals were detected in all three PD samples (PD1-PD3), whereas nothing remained in the control sample (CT; brain extract from an individual, in which a synucleinopathy was excluded). (b) ThT-binding kinetics of recombinant aSyn with or without nominated PMCA products as seeds. ThT-intensities for the three PD samples (PD1-PD3) were averaged and used to derive error bars. (c) Relative ThT fluorescence after seeding with different PMCA products. Error bars indicate standard deviation over three fluorescence measurements for each sample. 
The Korean collaborators characterized the PMCA end product seeded aSyn aggregates by Western blot analysis after proteinase $\mathrm{K}(\mathrm{PK})$ digestion with a monoclonal aSyn-specific antibody (BD Biosciences, \#610787) and monitored the seeded aggregation kinetics by thioflavin $\mathrm{T}$ binding assay as described by Jung et al. ${ }^{106}$.

Figure 3-4 a shows the amplification of PK-resistant aSyn aggregates in the case of PD patients (PD1, PD2 and PD3) using $0.5 \%$ (w/w) PMCA end products as seeds for seeded aggregation. The control brain extract (CT) PMCA end product in contrast did not result in the formation of PK-resistant aSyn aggregates, indicating the absence of competent aSyn seeds in the brain tissue. In addition, in panel b of Figure 3-4 the formation of ThT positive aSyn aggregates was monitored over time during the seeded aggregation amplification step. Again, the PMCA end product of control brain did not induce aSyn fibril formation, the ThT signal stayed low. The PMCA end products of the three PD patients however induce the formation of ThT-positive aSyn aggregates. Because recombinant aSyn in the absence of seeds did not form ThTpositive aSyn aggregates, de novo aggregation could be ruled out.

\subsubsection{Aggregation of high and low salt in vitro polymorphs}

Besides the amplification of aSyn form brain extracts, recombinant aSyn was additionally aggregated under two different, previously described buffer conditions through agitation ${ }^{117,122}$ but in the absence of PMCA-seeds. These two types of aSyn fibrils - obtained through de novo aggregation at ionic strengths of either $5 \mathrm{mM}$ (low salt; IsAsyn) or $200 \mathrm{mM}$ (high salt; hsAsyn) - differ in their conformations and neurotoxic phenotypes ${ }^{117,122}$. The de novo aggregated fibrils were subsequently used to seed aggregation of monomeric aSyn (unlabeled and ${ }^{15} \mathrm{~N}$-labeled). 


\subsection{Characterization of aSyn aggregates by EM}

Negative stain transmission electron microscopy (EM) with uranyl acetate was used to characterize the nature of aSyn aggregates seeded by PMCA end products for the different patients. For the high and low salt polymorphs (hsAsyn and IsAsyn), electron micrographs were already published by Bousset et al. ${ }^{122}$. In addition, EM was a valuable tool for quality control of the seeded aggregations as a yes/no readout for the fibrillization of aSyn.

\subsubsection{EM of aSyn aggregates amplified from patient brain material by PMCA}

The PMCA-seeded aSyn aggregates are genuine amyloid fibrils confirmed by negative-stain transmission EM. Figure 3-5 show representative micrographs for one patient of each type of synucleinopathy (PD1, MSA1 and DLB1). The aSyn fibrils have a diameter of $\sim 10$ to $12 \mathrm{~nm}$.



\section{MSA1}



\section{DLB1}

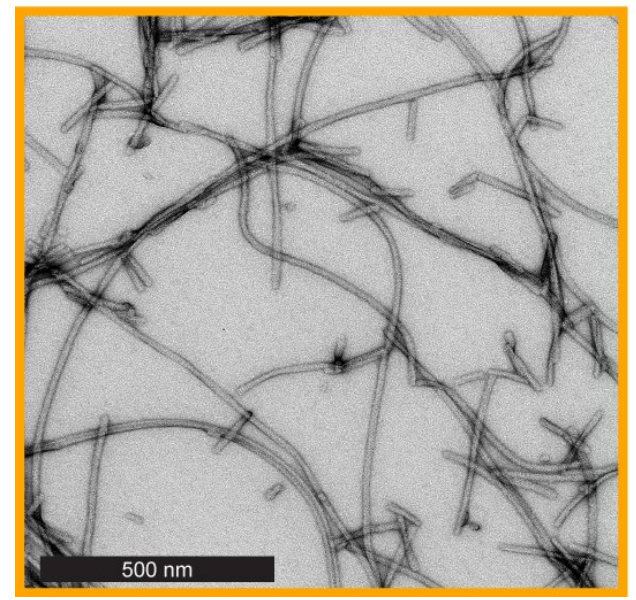

Figure 3-5. Electron micrographs of aSyn fibrils amplified from brain extracts.

Electron micrographs belong to fibrils amplified from brains extracts of PD, MSA and DLB. 
aSyn fibrils extracted from patients' brains are polymorphic with fibril diameters of $\sim 5$ and $\sim 10 \mathrm{~nm}^{4}$.

\subsubsection{EM pictures of high and low salt in vitro polymorphs}

The high salt polymorph, hsAsyn, shows fibrils with a diameter of $\sim 10$ to $12 \mathrm{~nm}$ as well as small single filaments of $\sim 5$ to $6 \mathrm{~nm}$.

\section{hsAsyn}

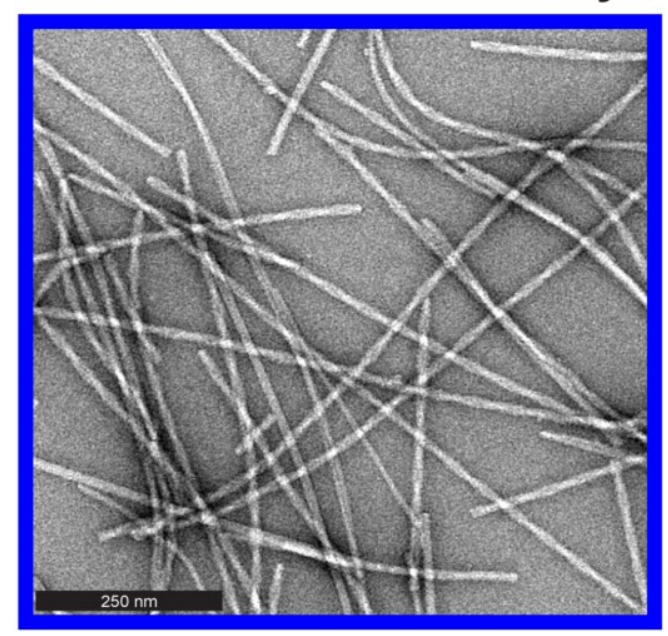

IsAsyn

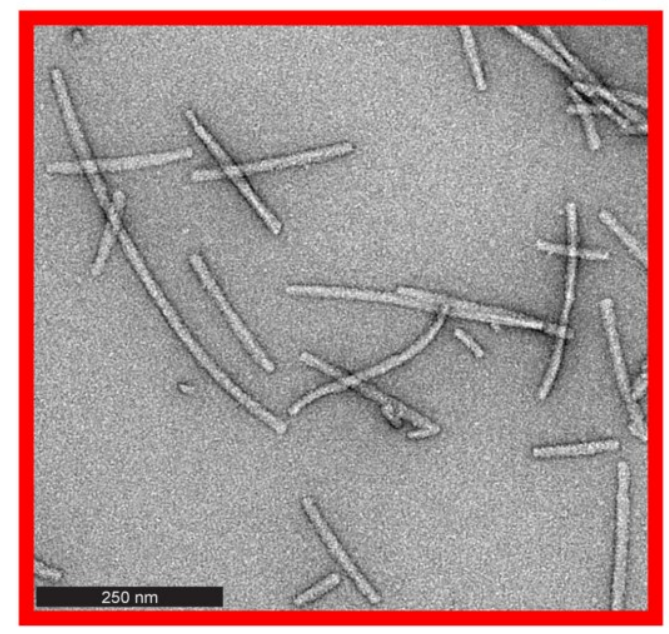

Figure 3-6. Electron micrographs of aSyn in vitro polymorphs.

Electron micrographs of the two in vitro aSyn polymorphs hsAsyn $(50 \mathrm{mM} \mathrm{Tris-} \mathrm{HCl}, 150 \mathrm{mM}$ $\mathrm{KCl}, \mathrm{pH} 7.5,0.02 \% \mathrm{NaN} 3)$ and IsAsyn (5 mM Tris- $\left.\mathrm{HCl}, \mathrm{pH} 7.5,0.02 \% \mathrm{NaN}_{3}\right)$.

\subsection{Secondary structure analysis of aSyn fibrils by CD spectroscopy}

Having obtained genuine aSyn fibrils in appearance by TEM characterization, circular dichroism (CD) was used to characterize their secondary structure. Figure 3-7 summarizes the results. The CD spectra show an amyloid $\beta$-sheet structure with a characteristic minimum at $\sim 220 \mathrm{~nm}$ and a maximum at $\sim 195 \mathrm{~nm}$. Random coil conformation of non-aggregated intrinsically disordered monomeric aSyn in solution was ruled out in the absence of the characteristic minimum at $\sim 200 \mathrm{~nm}$. 

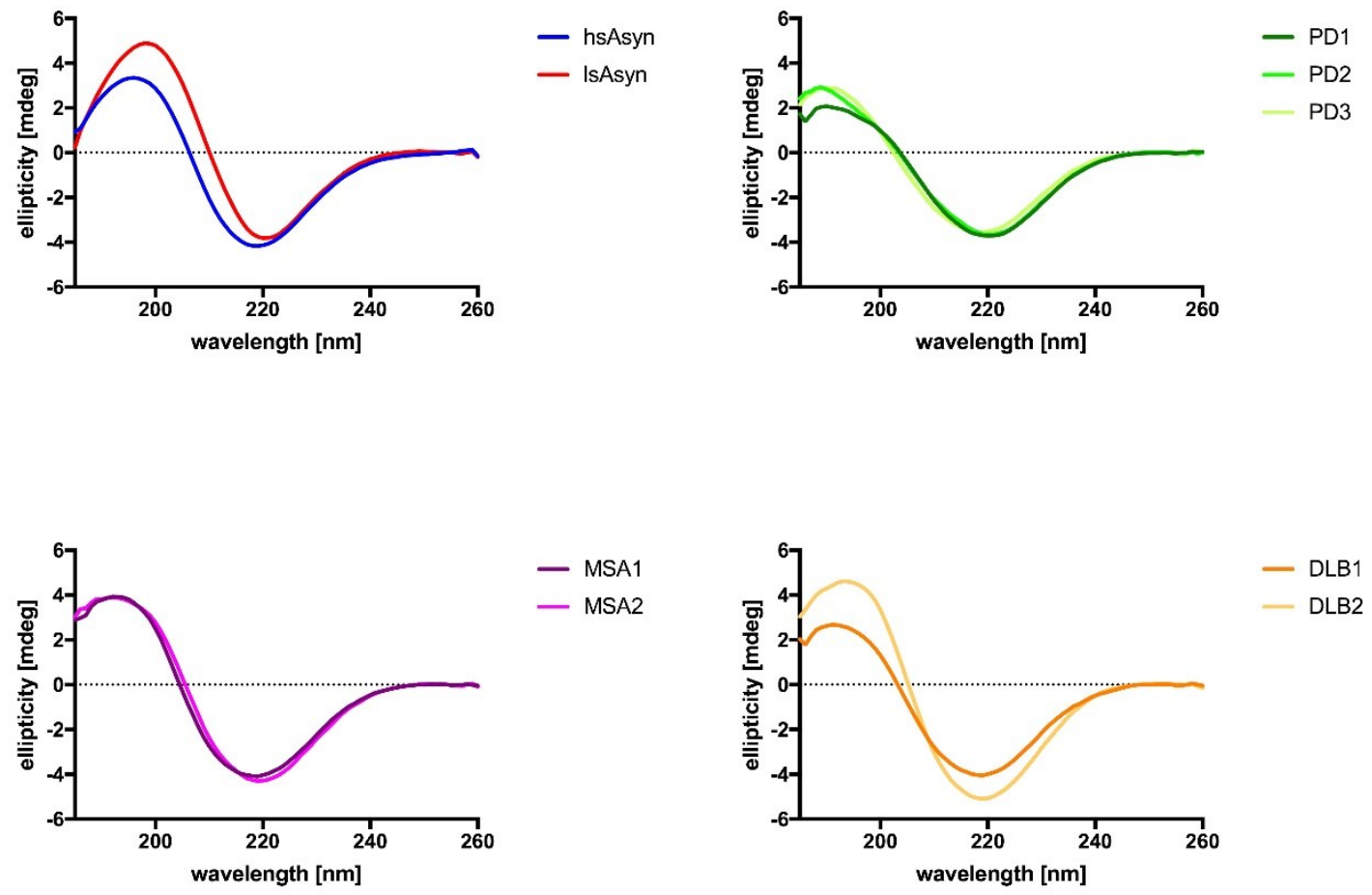

Figure 3-7. CD spectra of aSyn fibrils.

CD spectra of the two in vitro aSyn polymorphs (hsAsyn/blue, IsAsyn/red) and amyloid fibrils amplified from brain extracts of different patients (PD/green, MSA/purple, DLB/orange).

\subsection{Detection of conformational differences by fluorescent dye binding}

Fluorescent dyes that bind to specific amyloid conformations are able to differentiate prion strains and determine intersubject variability of amyloid- $\beta$ aggregates in Alzheimer's disease ${ }^{130,131}$. To investigate the presence of conformational differences between aSyn fibrils, fluorescence measurements in the presence of the amyloidbinding dyes curcumin, FSB and HS-68 $8^{130,132}$ were performed.

\subsubsection{Curcumin binding to aSyn fibrils}

Figure 3-8 $a$ and $b$ show the fluorescence spectrum of curcumin upon binding to aSyn fibrils for two independent measurements of the same sample fibril harvest. Both spectra show two local maxima, one at $\sim 519 \mathrm{~nm}$ and the other at $\sim 593 \mathrm{~nm}$. When normalized to the maximum observed for the hsAsyn polymorph at $\sim 519 \mathrm{~nm}$, a clear difference in the intensity of the maximum at $\sim 593 \mathrm{~nm}$ was evident between hsAsyn and IsAsyn. 
a



b

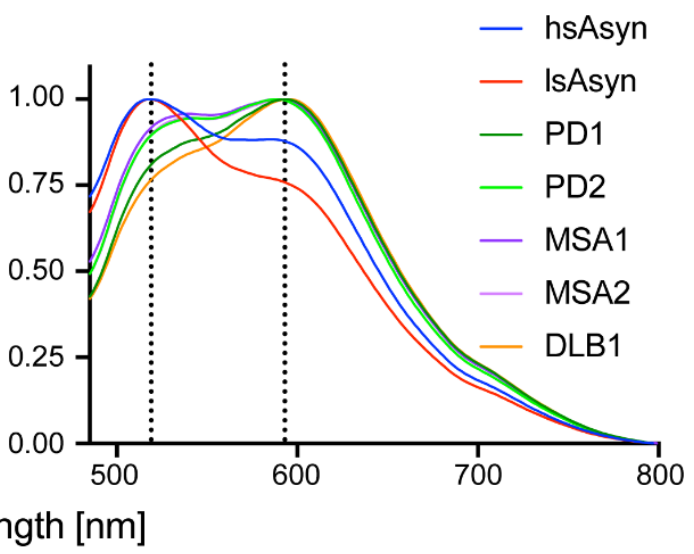

C

wavelength [nm]



Figure 3-8. Curcumin binding to aSyn fibrils.

(a) and (b): Normalized fluorescence spectra of curcumin in the presence of two in vitro aSyn polymorphs (hsAsyn/blue, IsAsyn/red) and amyloid fibrils amplified from brain extracts of different patients (PD/green, MSA/purple, DLB/orange). (c) Two-dimensional analysis of fluorescence properties of curcumin in the presence of different aSyn fibrils. The position of the global maximum is correlated to the ratio of fluorescence intensities at $\sim 519 \mathrm{~nm}$ and $\sim 593 \mathrm{~nm}$ (see panel $a$ and b). For each sample, fluorescence measurements were performed independently two times (two symbols each; representing intrinsic variability in the fluorescence measurements).

While the in vitro polymorphs displayed the maximal curcumin fluorescence at $\sim 519$ $\mathrm{nm}$, this maximum was shifted to $\sim 593 \mathrm{~nm}$ for the brain extract-amplified fibrils (Figure 3-8 a,b). Correlation of the position of the global maximum with the intensity ratios of the curcumin fluorescence at $\sim 519 \mathrm{~nm}$ and $\sim 593 \mathrm{~nm}$ further distinguished brain extractamplified fibrils from the in vitro polymorphs (Figure 3-8 c). Differences in the fluorescence spectra of samples amplified from PD, MSA and DLB brain extracts were also evident, including differences in the normalized curcumin fluorescence at $\sim 519$ 
$\mathrm{nm}$ (Figure 3-8 c). The raw absolute fluorescence is shown exemplarily for the graph of Figure 3-8 b in Figure 3-9.

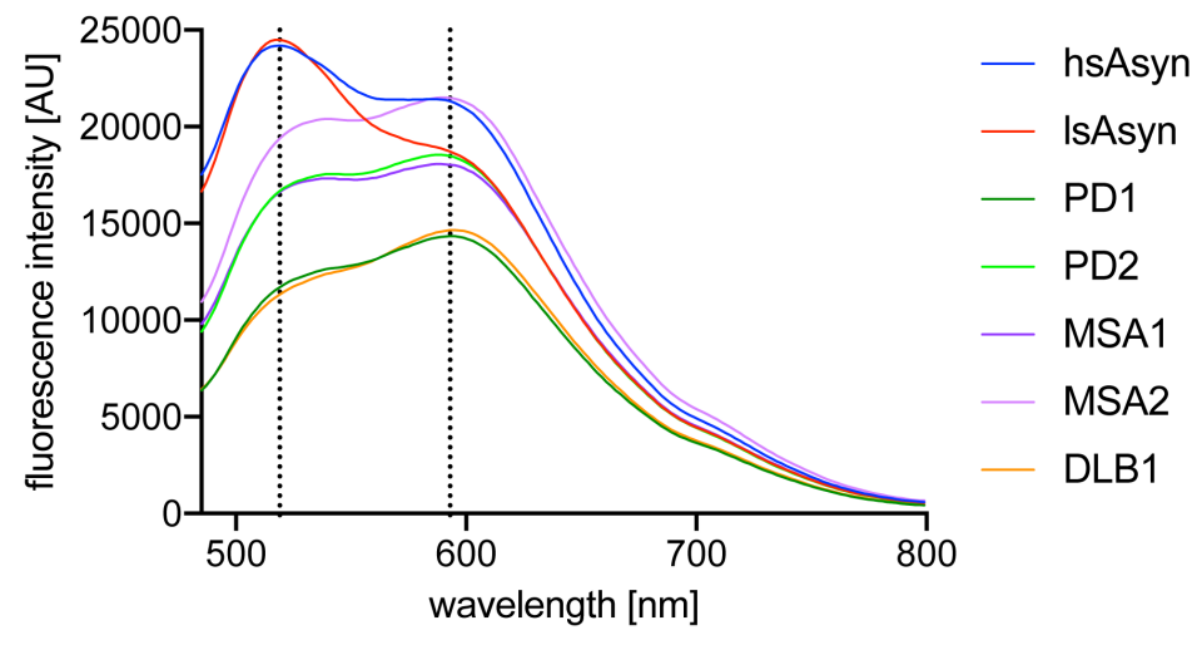

Figure 3-9. Absolute fluorescence of curcumin binding to aSyn fibrils.

Data shown here refers to the normalized graph of Figure 3-8b.

\subsubsection{FSB and HS-68 binding to aSyn fibrils}

In agreement with curcumin/aSyn fibril interactions, FSB also shifts the fluorescence emission maximum to longer wavelength in case of the brain extract-amplified fibrils. Differences in the fluorescence spectra of samples amplified from PD, MSA and DLB brain extracts were also evident in the absolute fluorescence intensities of FSB, see Figure 3-10 a. HS-68, Figure 3-10 b, shows stronger interaction with the high salt polymorph, hsAsyn. The fluorescent dyes indicated the presence of differences in the fibril conformations, mainly pointing out at differences between the in vitro polymorphs and the brain extract-amplified fibrils. In addition, the binding studies hinted towards conformational differences within the brain extract-amplified fibrils. 
a
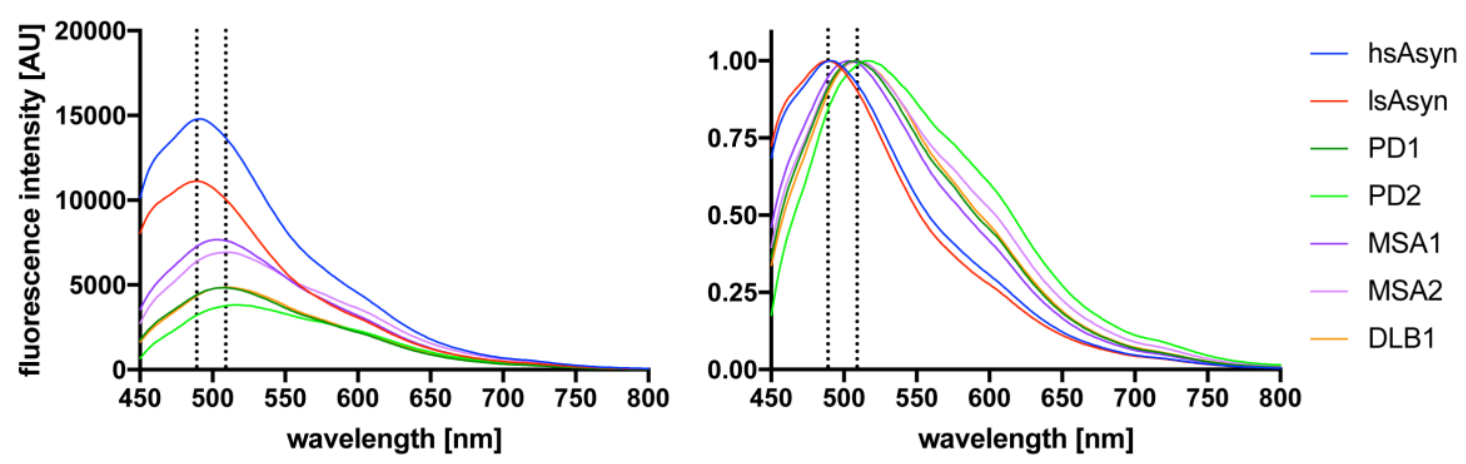

b
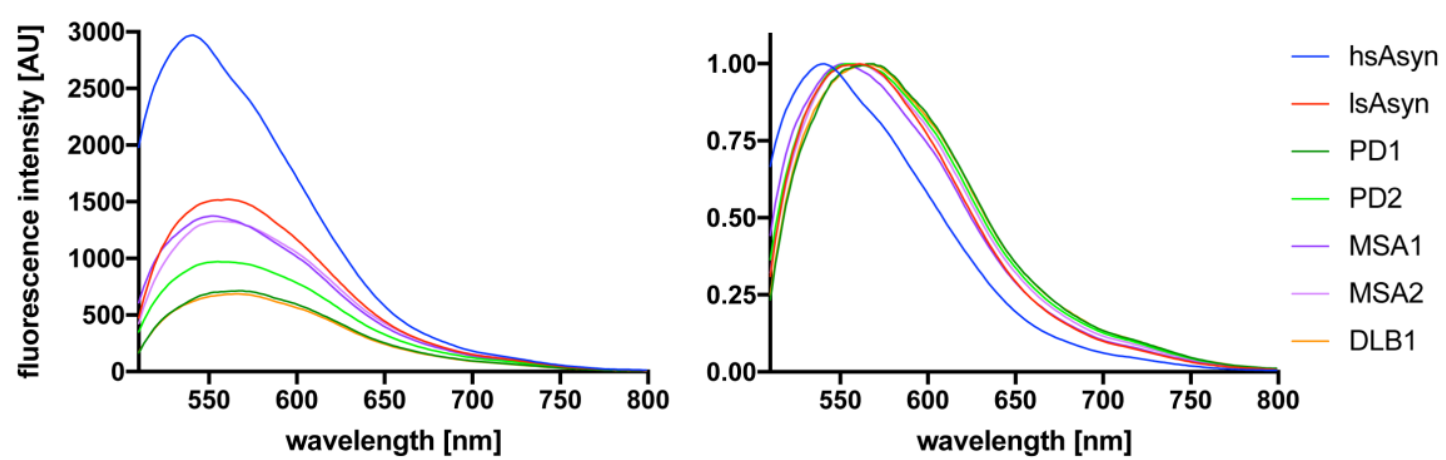

Figure 3-10. FSB and HS-68 binding to aSyn fibrils.

Fluorescence spectra of FSB (a) and HS-68 (b) in the presence of different amyloid fibers. Absolute and normalized fluorescence intensities are shown in the left and right column, respectively. The fluorescence maximum of FSB is shifted to longer wavelength in the presence of amyloid fibrils amplified from patient material (PD/green, MSA/purple, DLB/orange) when compared to the two in vitro polymorphs hsAsyn (blue) and IsAsyn (red). The average wavelengths of the fluorescence maxima of the two in vitro polymorphs $(\sim 489 \mathrm{~nm})$ and the aSyn fibrils amplified from patient brain extracts $(\sim 509 \mathrm{~nm})$ are marked by two dashed, vertical lines.

\subsection{Hydrogen-deuterium exchange coupled to NMR}

In order to gain insight into the conformational differences between different aSyn fibrils, hydrogen-deuterium (HD) exchange studies were conducted. When HD exchange studies are combined with methods of rapid fibril dissociation and liquidstate NMR spectroscopy, deuterium incorporation levels can be determined with single-residue resolution ${ }^{162-164}$. In the following sections the method is explained in detail, showing an in-depth analysis for a high salt polymorph. 


\subsubsection{Assignment of aSyn resonances in dissociation buffer}

An important prerequisite was the assignment of 131 (including prolines) out of 140 residues of aSyn in guanidinium thiocyanate dissociation buffer (4 M GdSCN, $0.4 \%$ formic acid, $\mathrm{pH}$ 2.5) with the help of $3 \mathrm{D} \mathrm{HNCO}, \mathrm{HN}(\mathrm{CA}) \mathrm{CO}, \mathrm{HNCA}, \mathrm{HN}(\mathrm{CO}) \mathrm{CA}$ and HNCACB experiments as described in section 2.15.1 - Resonance assignment and summarized in Appendix: 7.2. On one hand those assignments are essential for the analysis of HD exchange experiments and the calculation of initial protonation levels, on the other hand they allow analysis of the secondary structure of aSyn in the respective dissociation buffer. The presence of secondary structure elements would indicate intra- and potentially also intermolecular hydrogen bonds affecting the backexchange processes. In order to rule out this possibility, the secondary chemical shift differences to random coil NMR chemical shifts corrected for sequence-dependence ${ }^{161}$ are reported here for $\alpha$-carbon- $(\mathrm{C} \alpha)$, carbonyl- $\left(\mathrm{C}^{\prime}\right)$ and $\beta$-carbon $(\mathrm{C} \beta)$ resonances of aSyn.

a



C

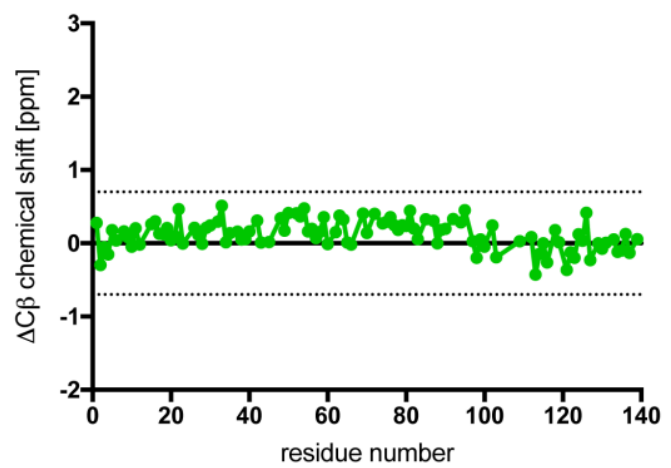

b

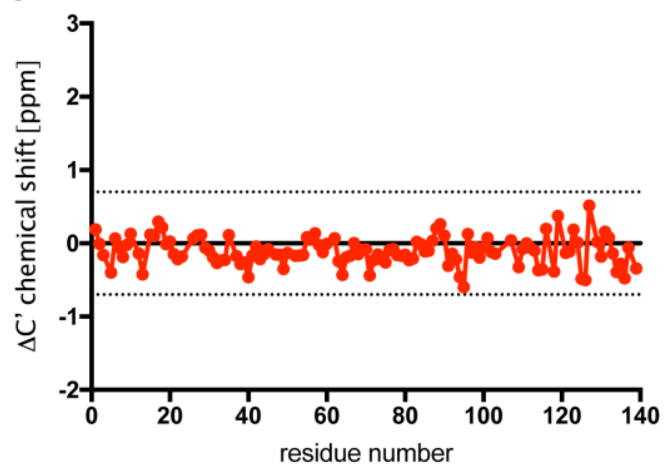

Figure 3-11. Secondary chemical shift differences.

(a) $C \alpha-$, (b) C'- and (c) $C \beta$-chemical shift differences. 


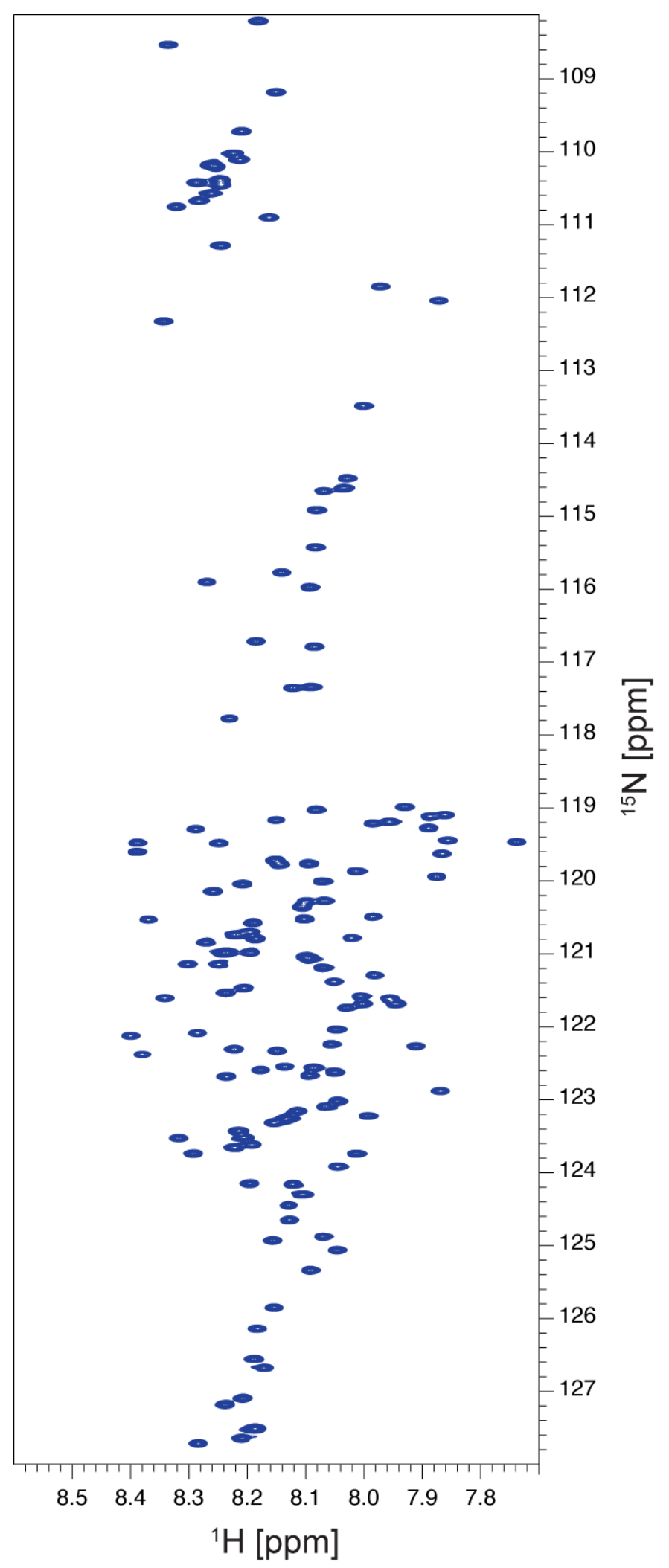

Figure 3-12. HSQC spectrum of aSyn in dissociation buffer.

Temp.: $276 \mathrm{~K}, 800 \mathrm{MHz}, 600 \mu \mathrm{M}$ aSyn in dissociation buffer (4 M GdSCN, 0.4\% formic acid, $\mathrm{pH} 2.5)$. 
The shift differences are small (Figure 3-11 a,b and c), therefore the presence of secondary structure elements could be ruled out. aSyn in the guanidinium thiocyanate dissociation buffer (Figure 3-12) behaves as a random coil and the buffer is suited for HD exchange measurements by NMR.

\subsubsection{Hydrogen-deuterium back-exchange in dissociation buffer}

Previous HD exchange studies conducted on in vitro aggregated aSyn fibrils established that a forward exchange time of 1.5 days allows robust determination of residue-specific protonation levels ${ }^{162,164}$. The data analysis from the acquisition of realtime HSQC spectra to the calculation of residue-specific protonation levels is shown here for in vitro seeded aSyn fibrils under high salt buffer conditions $(50 \mathrm{mM} \mathrm{Tris-HCl}$, $150 \mathrm{mM} \mathrm{KCl}, \mathrm{pH} 7.4,0.02 \% \mathrm{NaN}_{3}$ ), hsAsyn.

The forward-exchanged hsAsyn pellet was taken from the $-80{ }^{\circ} \mathrm{C}$ storage, thawed and resuspended in dissociation buffer, followed by sample transfer to the NMR tube. All steps were performed on ice to quench the back-exchange kinetics. The NMR sample was quickly inserted into a pre-shimmed and temperature-equilibrated (276 K) $800 \mathrm{MHz}$ cryoprobe-equipped NMR spectrometer and a series of HSQC spectra were recorded monitoring in real-time the back-exchange in dissociation buffer $(\mathrm{H}: 40 \%, \mathrm{D}: 60 \%)$ as described in detail in the Material and methods section.

Figure 3-13 shows on the left side in blue the initial HSQC spectrum of the realtime series, the absolute intensities by height are a close approximation of the intensity values at $t_{0}$ that report the initial protonation levels for each residue after forward exchange. The light blue spectrum on the right side of Figure 3-13 shows the final HSQC spectrum of the HSQC series, representing the fully back-exchanged hsAsyn spectrum where the intensities report the protonation level of the dissociation buffer.

The series of HSQC spectra was converted into an intensity table using the resonance assignment for aSyn in dissociation buffer. Peaks were picked in the final HSQC spectrum and centered to the maximum peak height. Using nmrPipe's seriesTab script, the intensity values for each residue of every HSQC plane were extracted. The intensity values of the final HSQC of each aSyn residue were set arbitrarily to 1 and the series normalized accordingly. Knowing the initial time delay from pellet resuspension to the start of the first HSQC as well as the duration of each HSQC pulse program, the table can be plotted against the absolute time axis shown in Figure 3-14. To back-calculate the initial intensity level of each aSyn residue, the time curves were fitted to a single exponential decay and the relative intensity values for $t_{0}(t=0$ hours $)$ were reported. The fit is shown as a continuous line in Figure 3-14. 


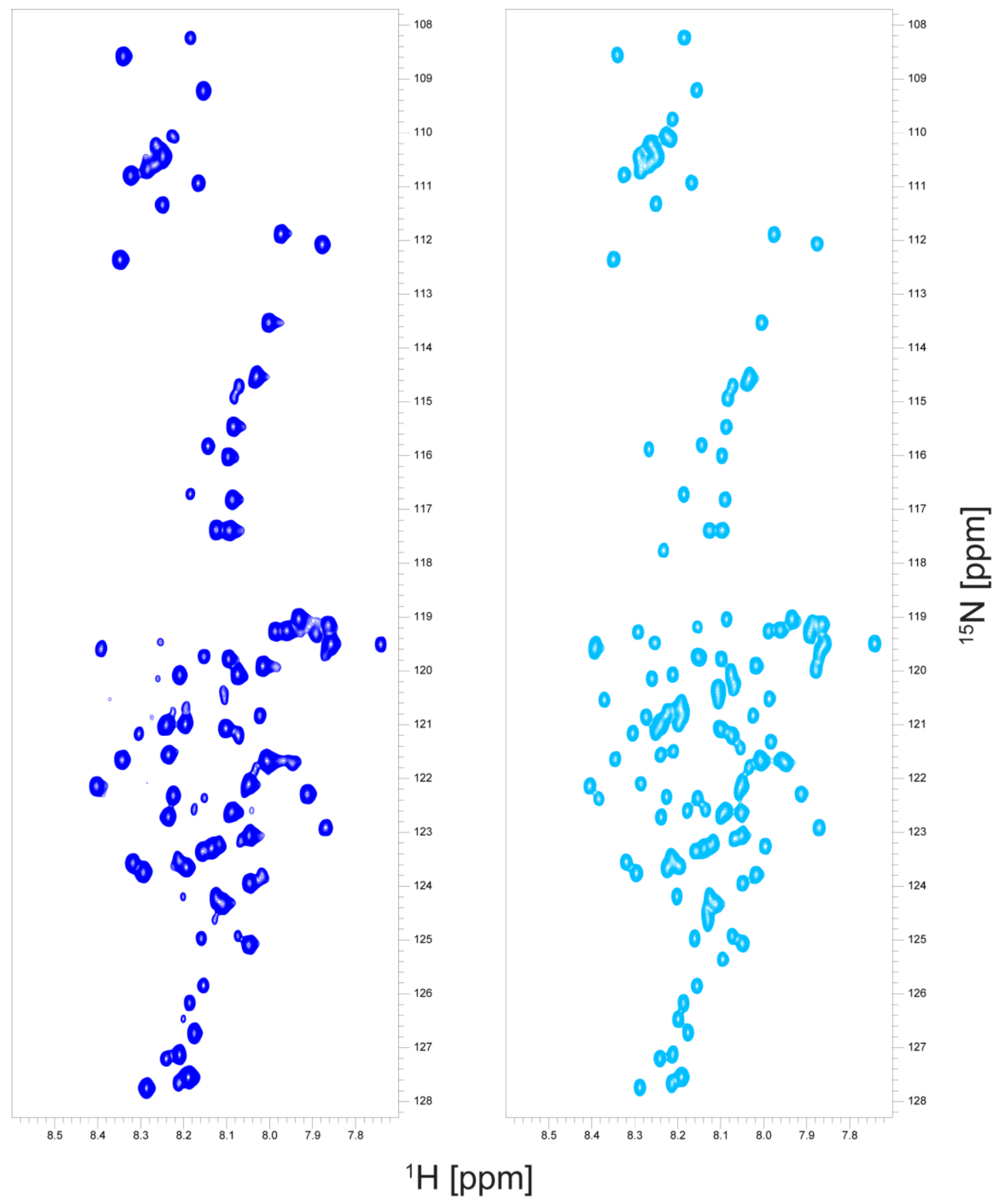

Figure 3-13. HD back-exchange monitored by HSQCs in real-time.

Full ${ }^{1} \mathrm{H}-{ }^{15} \mathrm{~N}$ HSQC spectra recorded after fibril dissociation at the beginning (left) and end (right) of the back-exchange period for the hsAsyn polymorph. 

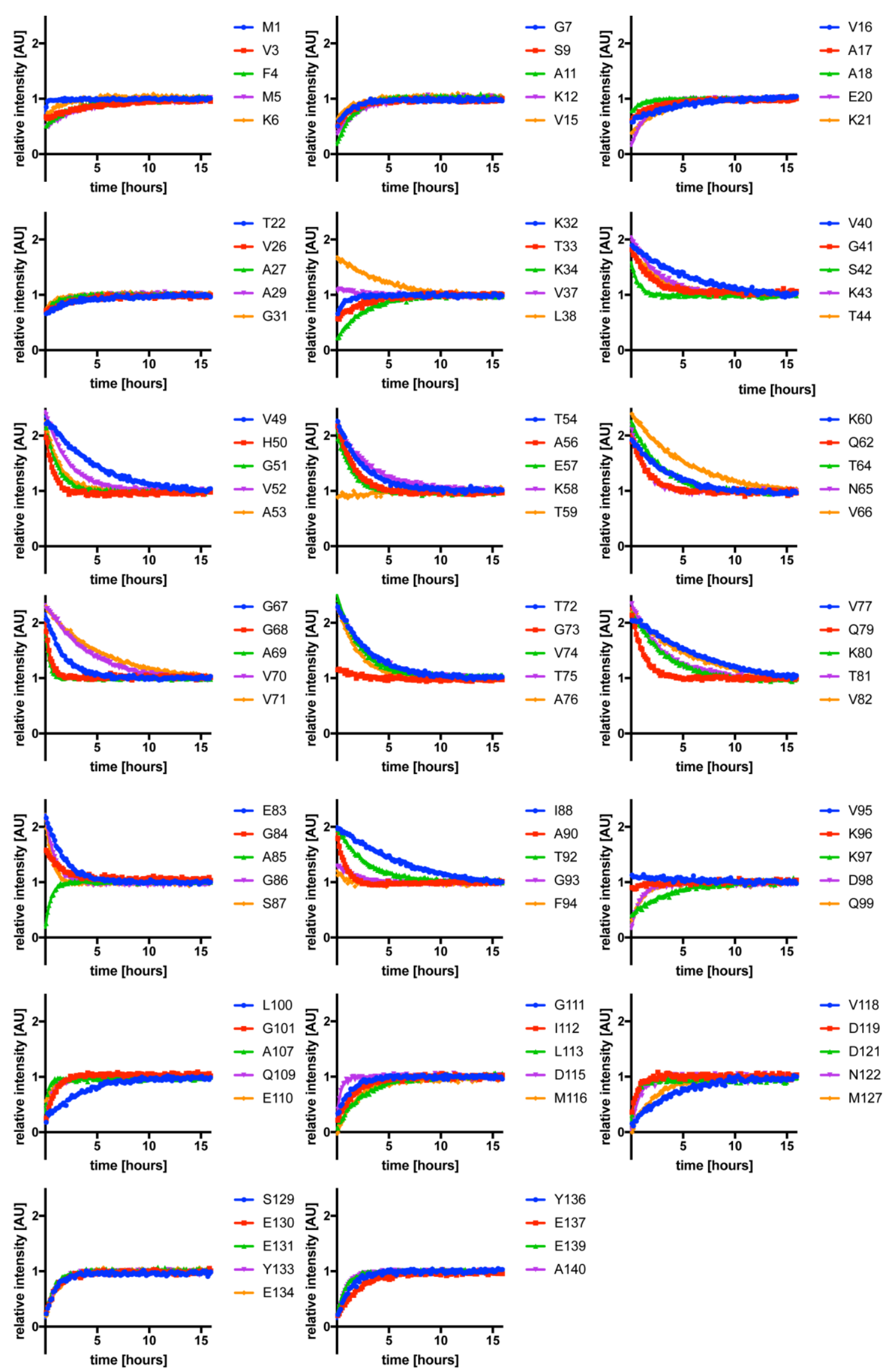

Figure 3-14. Back-exchange curves of hsAsyn polymorph.

99 non-overlapping HSQC cross peaks were included in analysis. 
All hsAsyn residues converged within the duration of the real-time measurement to the final normalized intensity value 1 . Differences in the rate constant are sequence- and residue type-dependent. The upper part of Figure 3-15 shows the back-fitted intensity values at $\mathrm{t}_{0}$. The lower part the absolute protonation levels in $\%{ }^{1} \mathrm{H}$.
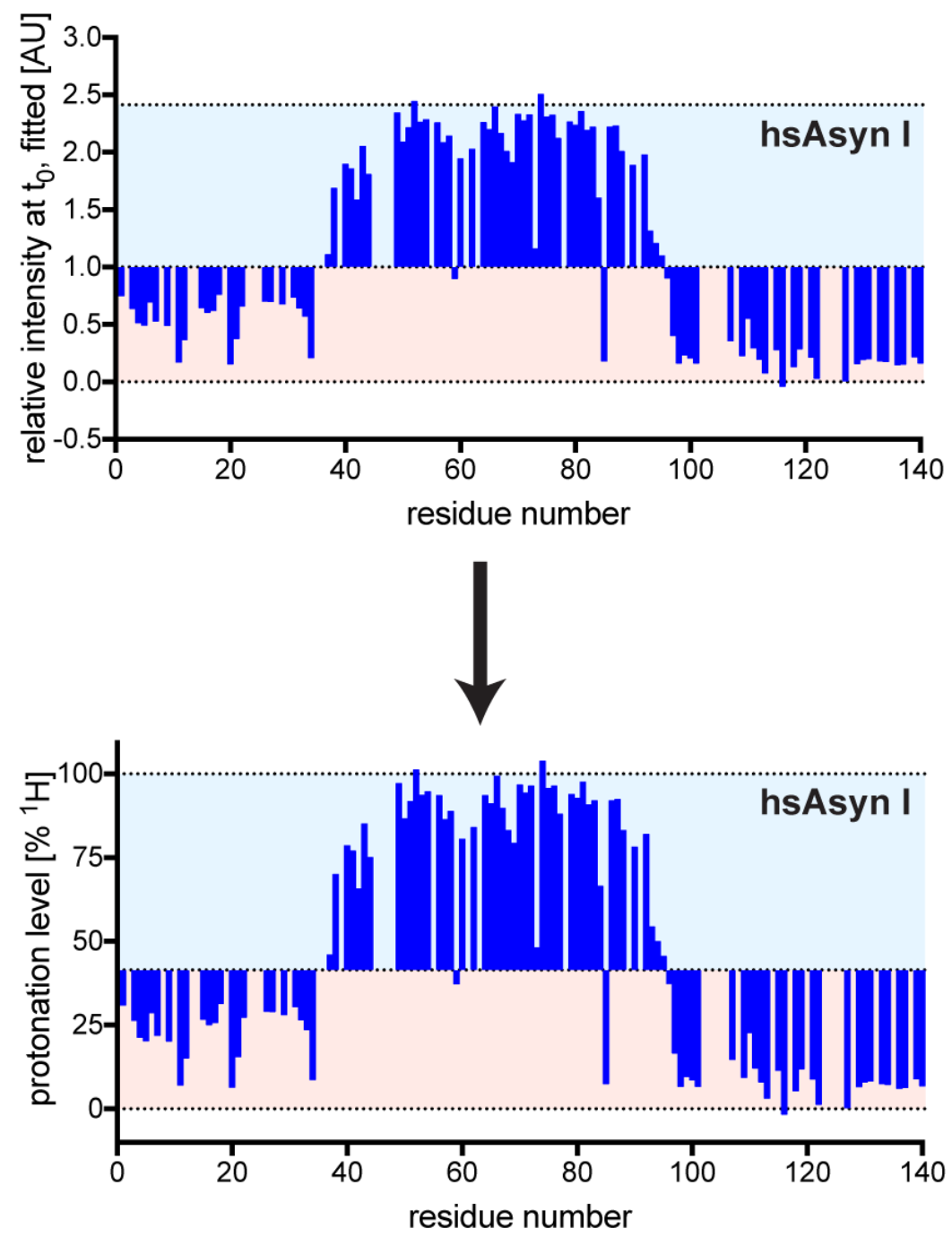

Figure 3-15. Data transformation from intensities to absolute protonation levels.

Arrow indicates transformation operation. The average of the sequence-independent residues of highest intensity is calculated and set as a protonation level of $100 \%{ }^{1} \mathrm{H}$. The relative intensity 1 reports the absolute protonation level of the dissociation buffer, in case of hsAsyn $59 \% \mathrm{D}_{2} \mathrm{O}$.

The structural implications of the detected protonation levels after HD exchange are discussed in the next section. 
3.7 HD exchange profile of the in vitro high and low salt polymorphs

3.7.1 HD exchange profile of the high salt in vitro polymorph

a

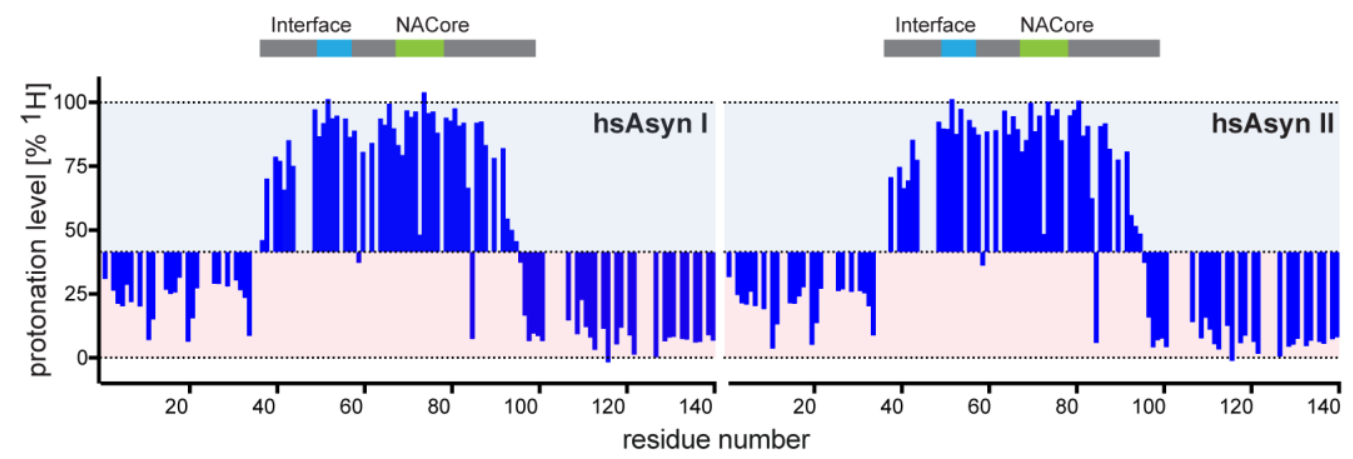

b



C

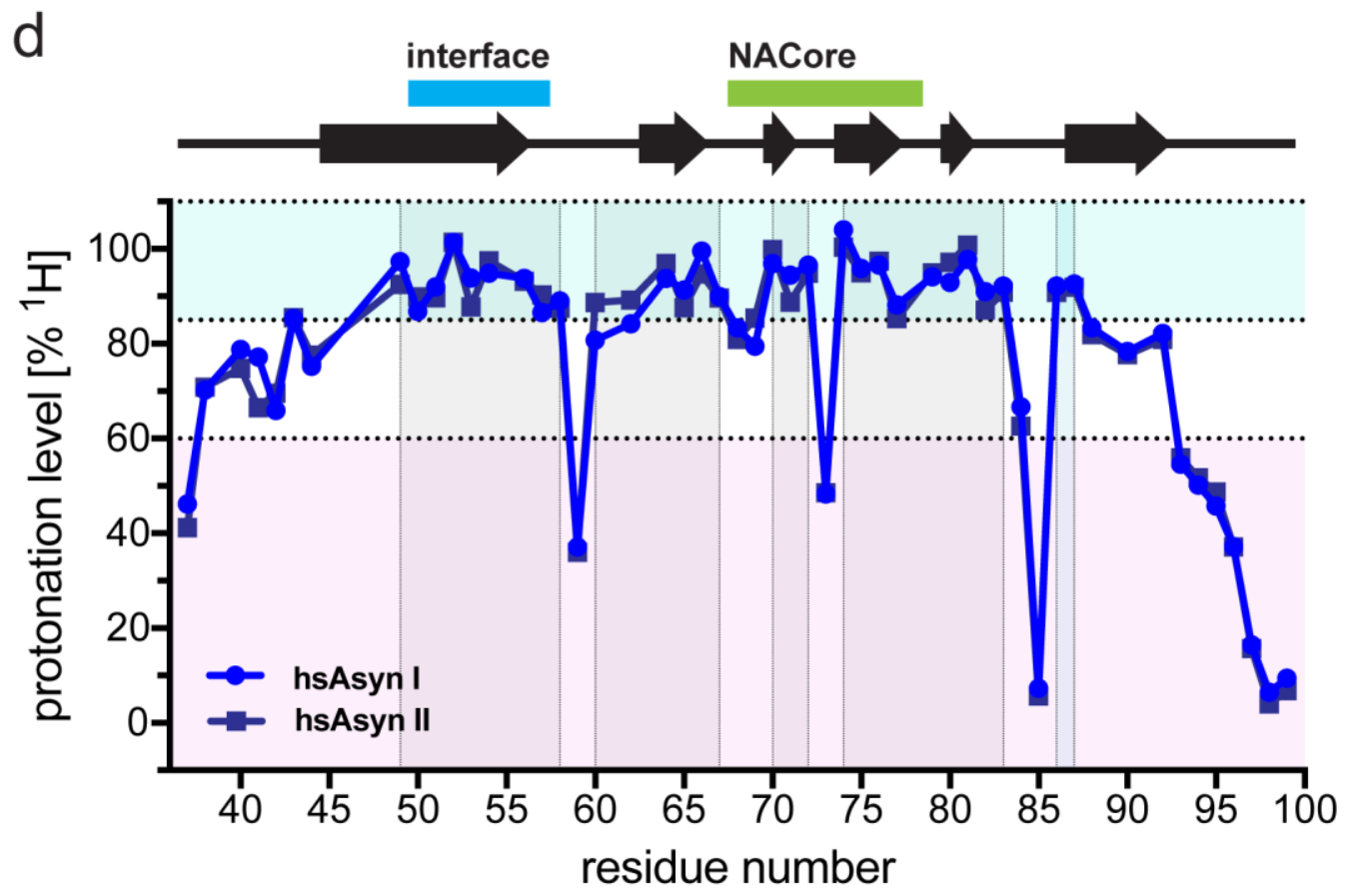


Figure 3-16. Residue-specific protonation levels in high salt polymorph (hsAsyn) fibrils by HD exchange.

(a) HD-exchange profiles of two independently seeded hsAsyn fibrils using the same de novo aggregates as seeds, labeled as hsAsyn I and II. (b) Selected region from ${ }^{1} \mathrm{H}-{ }^{15} \mathrm{~N}$ correlation spectra recorded after fibril dissociation at the beginning (left) and end (right) of the backexchange period for hsAsyn I fibrils, in detail see Figure 3-13. (c) Time-dependent backexchange of selected residues (E20, T75 and A90) for hsAsyn I fibrils, in detail see Figure 3-14. (d) Detailed HD exchange profile of the in vitro polymorph (hsAsyn I in blue and hsAsyn II in dark blue) within the region that forms cross- $\beta$-sheets in the cryo-electron microscopy structure of hsAsyn (PDB code: 6A6B). NACore is the most hydrophobic part of the aSyn sequence and the interface between two aSyn filaments as seen in the PDB of 6A6B is marked, both in panel a and d above.

In agreement with previous data ${ }^{162,164}$, it was found for the high salt polymorph that the fastest solvent exchange occurs in residues located in the $\sim 40 \mathrm{C}$-terminal residues for which less than $25 \%$ of amide protons remained after 1.5 days of forward exchange in $\mathrm{D}_{2} \mathrm{O}$ (Figure 3-16 a). Extensive incorporation of deuterium was also observed for the first 36 residues at the N-terminus of aSyn (Figure 3-16 a). In contrast, residues L38V92 retained a large fraction of their amide protons after exchange in $\mathrm{D}_{2} \mathrm{O}$ (Figure 3-16 d), indicating that these amide protons participate in hydrogen bonds. The hydrogenbonded core of hsAsyn fibrils as determined by HD was consistent with those defined by solid-state NMR and cryo-electron microscopy for wild-type aSyn and aSyn(1$121)^{133-135}$. Figure 3-16 a-d show that protonation levels within the aSyn amyloid core were not uniform. Several residues within the region K43-S87 displayed little solvent exchange, with other residues retaining only $\sim 60-85 \%$ of their amide protons (i.e. L38T44, K60, Q62, G68-A69, G84, I88-T92) or even exchanging more than $60 \%$ (i.e. V37, T59, G73, A85, G93-Q99).

The structure of the hsAsyn in vitro polymorph (identical buffer conditions as used in the current study) has been recently determined by cryo-electron microscopy ${ }^{135}$. Figure 3-17 a shows the protonation levels determined by HD exchange in context of the cross- $\beta$-sheet structure of hsAsyn. Fast solvent exchange was undertaken by V37-T44 and G93-Q99, which are located in the N- and C-terminal parts, respectively, and are not surrounded by additional cross- $\beta$-sheet structure (Figure 3-17 a,b). A connection between solvent accessibility and differences in protonation levels was further supported by the finding that residues next to the hydrophilic channel ${ }^{133-135,173}$ displayed decreased protonation levels (Figure 3-17 a). In addition, faster solvent exchange for some turn residues (e.g. T59, A85; Figure 3-17 a) was observed, potentially due to lower hydrogen bond strength ${ }^{174}$. In contrast, high 
protonation levels for the residues A53-A56, which form the interface with a second

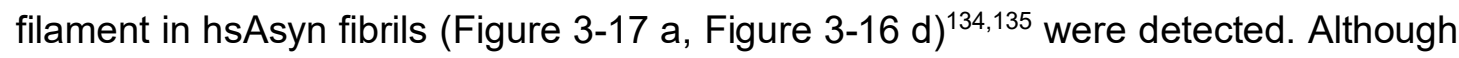
most residues within the amyloid core form hydrogen bonds to neighboring residues along the fibril axis (Figure 3-17 a,b), the analysis showed that HD exchange coupled to NMR spectroscopy provides a sensitive tool to characterize aSyn amyloid structure ${ }^{163}$.



b



Figure 3-17. HD exchange profile mapped on high-resolution cryoEM structure.

(a) Mapping of residue-specific protonation levels of hsAsyn I (as shown in blue in panel a of Figure 3-16) on the structure of the hsAsyn filament (PDB code: 6A6B). The interface to the second filament, which involves $A 53$, is marked. The scale bar indicates protonation levels relative to the minimum protonation level observed for V37-Q99. Residues with signal overlap were excluded from the analysis (shown in white). (b) Side view of (a). 
3.7.2 Robustness of the high salt in vitro polymorph HD exchange profile

Here it is shown that HD exchange profiles of de novo aggregated and seed-derived hsAsyn fibrils were highly similar (Figure 3-18 a). The amyloid structure was thus propagated during seeding. In addition, in vitro aggregation and seeding in another high salt buffer used for the aggregation of brain-extract seeded aSyn fibrils resulted in a very similar HD exchange pattern (Figure 3-18 b).
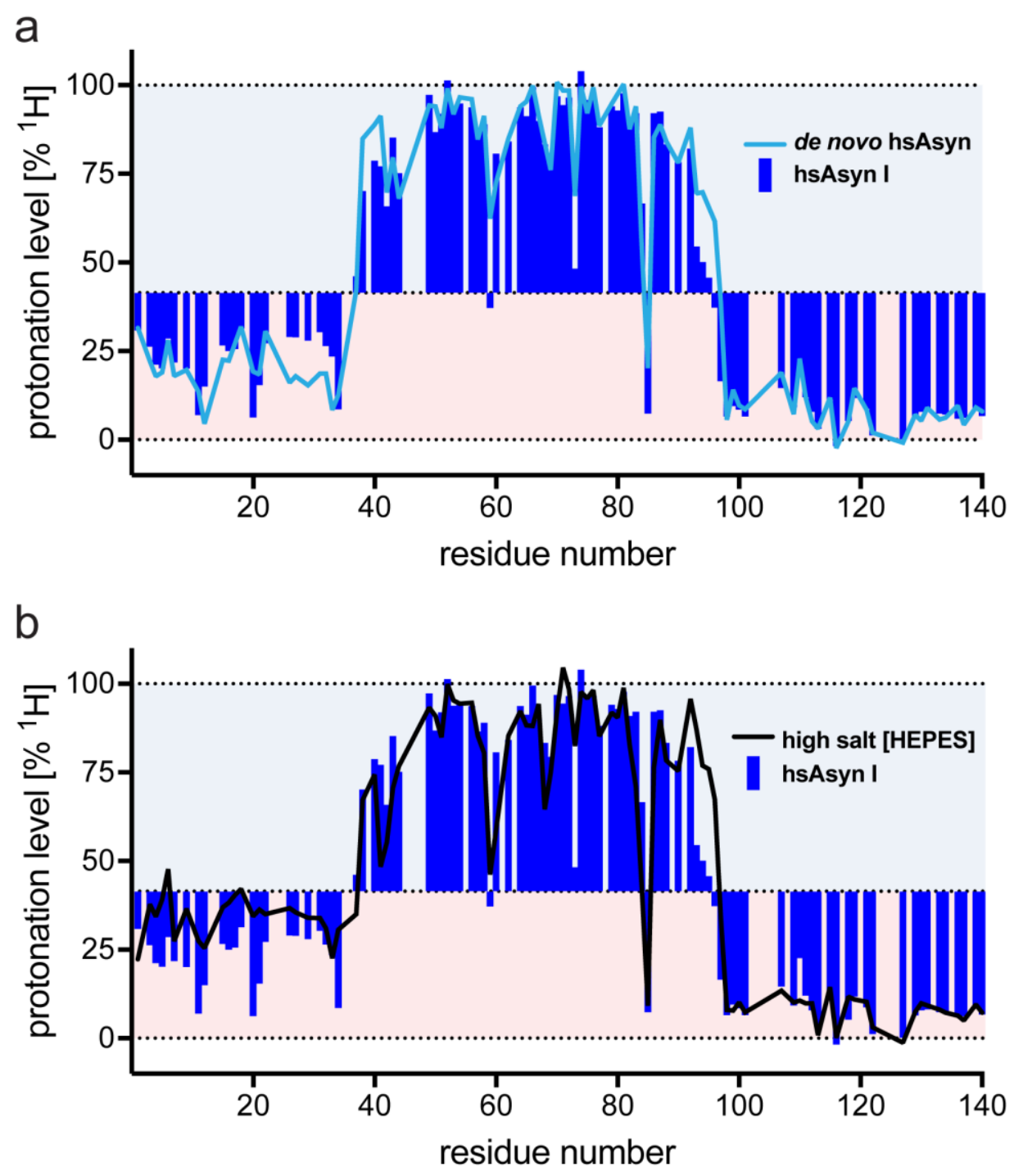

Figure 3-18. HD exchange profiles for in vitro high salt polymorph control experiments.

(a) Comparison of HD exchange profiles of de novo aggregated hsAsyn I fibrils (line) and hsAsyn fibrils obtained after seeding (blue bars). (b) Comparison of the HD exchange profile of hsAsyn I fibrils (blue bars; buffer: $50 \mathrm{mM}$ Tris- $\mathrm{HCl}, 150 \mathrm{mM} \mathrm{KCl}, \mathrm{pH} 7.5,0.02 \% \mathrm{NaN}_{3}$ ) with the $\mathrm{HD}$ exchange profile of aSyn fibrils obtained by aggregation in the aggregation buffer of brainextract seeded aSyn fibrils (black line; buffer: $50 \mathrm{mM}$ HEPES, $100 \mathrm{mM} \mathrm{NaCl}, \mathrm{pH} 7.4,0.02 \%$ $\mathrm{NaN}_{3}$ ). 
3.7.3 HD exchange profile of the in vitro low salt polymorph

Next, the HD exchange profile of the low salt in vitro polymorph (IsAsyn) was determined (Figure 3-19 a,b).

a



b



Figure 3-19. Residue-specific protonation levels in low salt polymorph (IsAsyn) fibrils by HD exchange.

(a) HD-exchange profiles of two independently seeded IsAsyn fibrils using the same de novo aggregates as seeds, labeled as IsAsyn I and II. (b) Time-dependent back-exchange of selected residues (E20, T75 and A90) for IsAsyn I fibrils.

In agreement with solid-state NMR spectroscopy ${ }^{122}$, the IsAsyn polymorph contains hydrogen-bonded structure in the $\sim 30 \mathrm{~N}$-terminal residues (Figure 3-19 a, red).

\subsubsection{Comparison of high- and low salt in vitro polymorphs}

Comparing the two HD exchange profiles to each other, differences in protonation levels in the central part were evident in the two in vitro polymorphs, Figure 3-20. In the IsAsyn polymorph, solvent exchange for residues V37-T44 and G93-K97 was decreased when compared to hsAsyn. The turn residues T59, K60, G68, A69, G73 and $\mathrm{A} 85$ also differed in the protonation levels. 
a

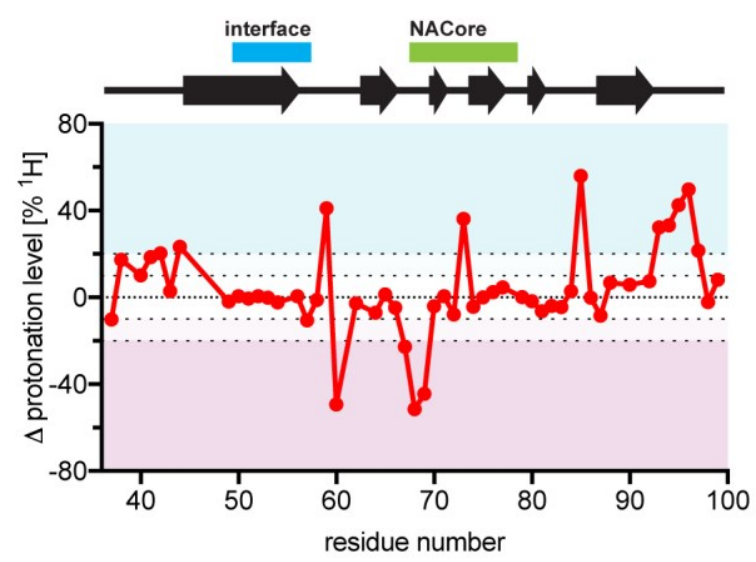

b

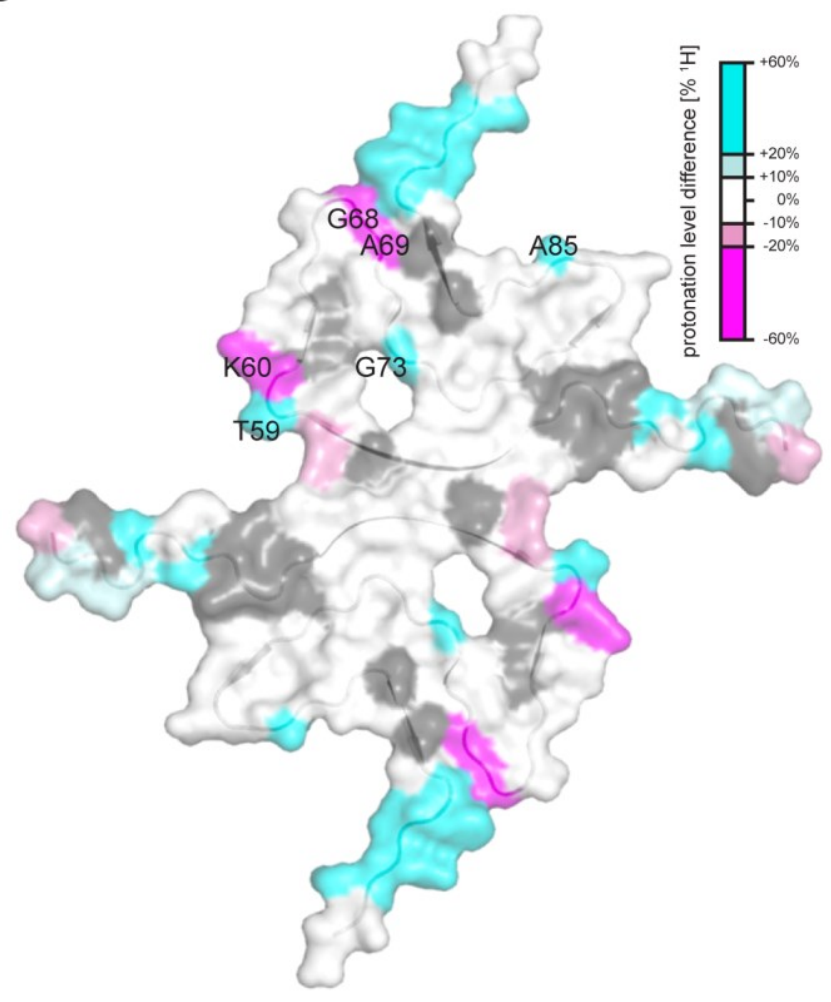

Figure 3-20. Comparison of HD exchange profile of high and low salt in vitro polymorphs.

(a) Residue-specific differences in the protonation levels of hsAsyn and IsAsyn in the region from V37 to Q99, mapped in (b) onto the core structure of hsAsyn fibrils (PDB code: 6A6B). Difference values were calculated on the basis of the average protonation levels observed for two independently seeded samples (hsAsyn I/II and IsAsyn I/II respectively, see Figure 3-16 a and Figure 3-19 a). NaCore and the interface of the aSyn filaments are marked.

Thus, changes in amyloid structure occur when aSyn is aggregated at a low ionic strength matching Bousset et al.'s observations ${ }^{122}$. 


\subsection{HD exchange profile of brain-extract seeded aSyn}

HD exchange profiles of fibrils amplified from three different PD patients were very similar (Figure 3-21 b, Figure 3-23 b). For residues A53-K80, PD-protonation levels were also similar to those in hsAsyn fibrils (Figure 3-16 a,c). In contrast, protonation levels of residues L38-T44 were lower in the amyloid fibrils amplified from PD patient brain extracts when compared to the protonation levels in the in vitro polymorphs (Figure 3-22 a). In addition, the protonation levels decreased significantly from E83, suggesting a loss of hydrogen bonds (or decrease in hydrogen bond strength) for E83K96 in PD-derived fibrils (Figure 3-22 a,b). Thus, amyloid fibrils that were amplified from PD patient brain extracts have a smaller hydrogen-bonded core than the two in vitro polymorphs hsAsyn and IsAsyn (Figure 3-22 a,b). This smaller core contains the most hydrophobic part of the aSyn sequence, the NACore region (G68-A78) which can form amyloid fibrils in isolation ${ }^{175}$. HD exchange profiles of aSyn fibrils amplified from brain extracts of three different PD patients were highly consistent (Figure 3-23 b). Two HD exchange profiles of aSyn fibrils amplified from brain extracts of individuals pathologically confirmed with MSA were also very similar to each other (purple bars in Figure 3-21 b), differences between MSA1 and hsAsyn I are shown for a selected region in the HSQC spectra (Figure 3-23 a). Disease-specific reproducibility was furthermore observed for the protonation levels of aSyn fibrils amplified from brain extracts of two DLB patients (orange bars in Figure 3-21 b). In addition, HD exchange profiles, which were obtained independently through seeding with PMCA-amplified fibrils from the same DLB patient, overlapped closely (Figure 3-23 c).

Because of the high reproducibility of the HD exchange studies, HD exchange patterns between different synucleinopathies (PD, MSA, DLB) were compared. Residues A18 to G93 displayed protonation levels above $50 \%$ in MSA-amplified fibrils (Figure 3-21 b), with V37-T44 and A85-G93 exchanging less rapidly when compared to PD-amplified fibrils (Figure 3-22 c,d). For DLB-amplified aSyn fibrils, protonation levels for residues A18-V48 and E83-G93 were in between those of PD and MSA (Figure 3-21 b). DLB- and MSA-amplified fibrils, however, had comparable protonation levels for A53-A56 (Figure 3-21 b, Figure 3-22 a), which were by 5-15\% lower than in PD-amplified fibrils (Figure 3-22 c,d). Because A53-A56 are located in the interface with a second filament in the hsAsyn structure (Figure 3-17 a, Figure 3-22 $\mathrm{d})^{134,135}$, the more rapid solvent exchange of A53-A56 in MSA- and DLB-amplified fibrils could be caused by differences in the quaternary structure of aSyn fibrils in MSA and DLB when compared to PD. 
a
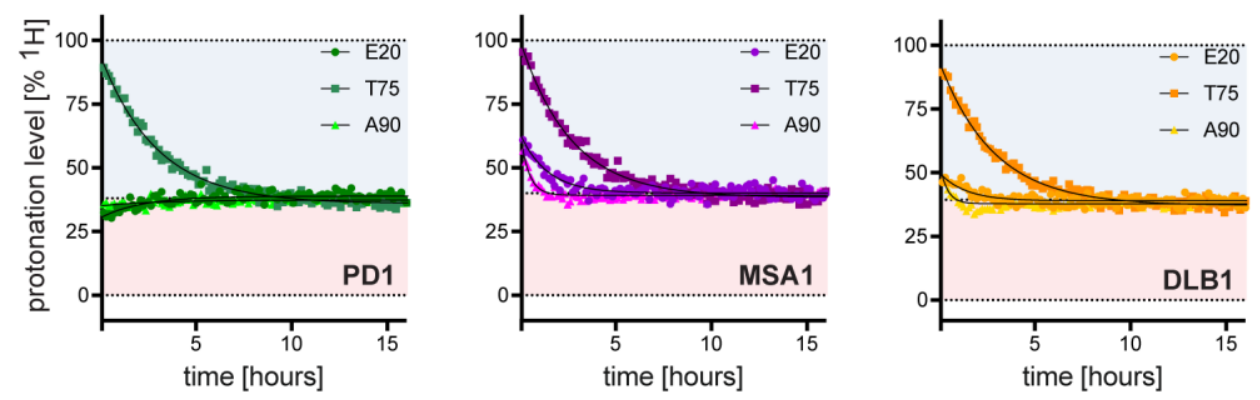

b

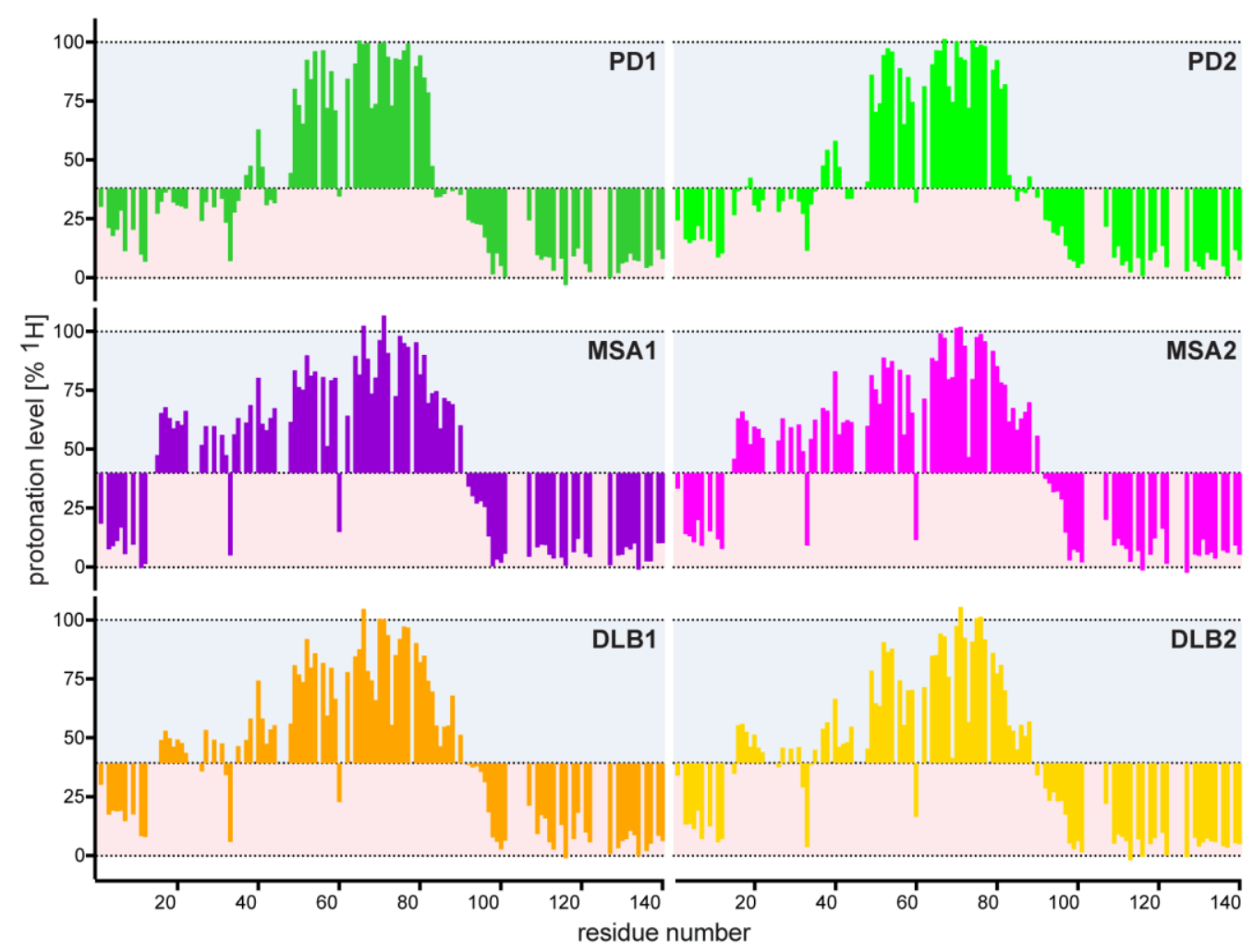

Figure 3-21. Residue-specific protonation levels of brain-extract seeded aSyn fibrils.

(a) Time-dependent back-exchange of patient-seeded aSyn fibrils of PD, MSA and DLB1 (E20, T75 and A90). (b) HD-exchange profiles of aSyn fibrils independently amplified from two individuals, which were pathologically confirmed with the same disease, are shown next to each other. 
a

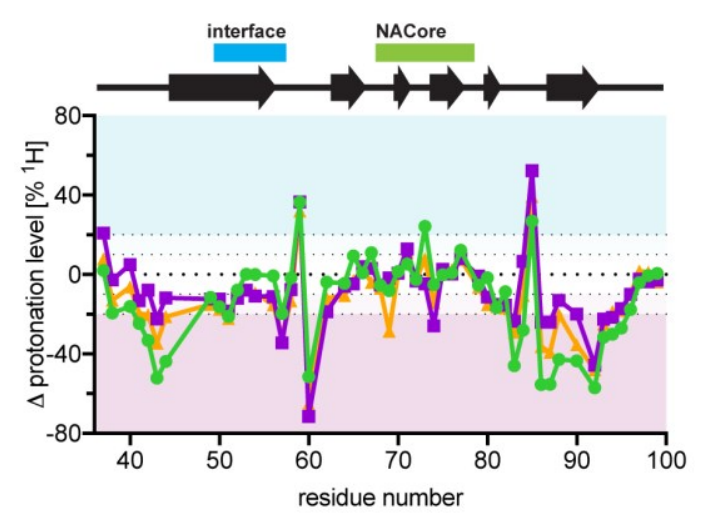

C

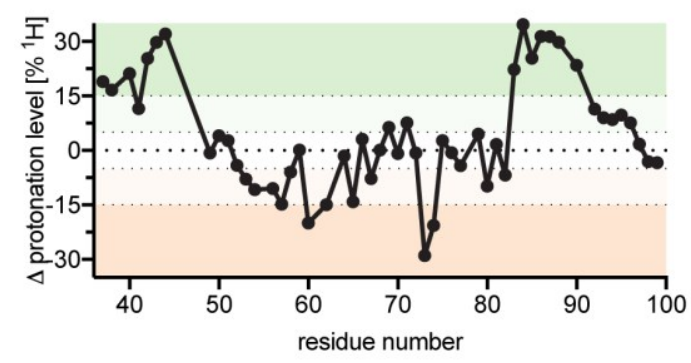

b



d

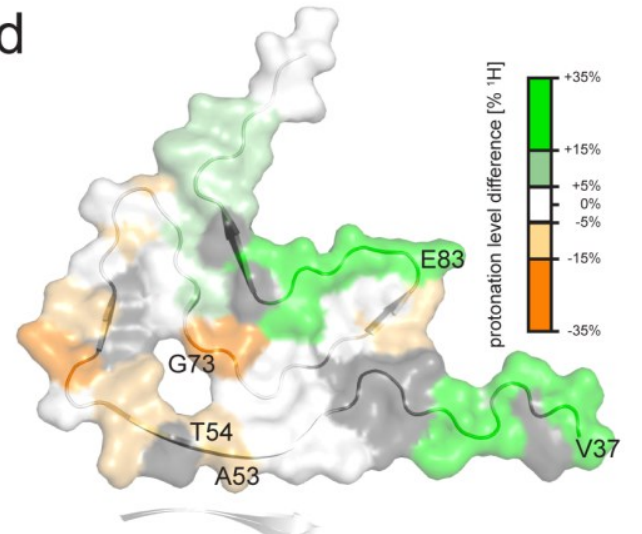

Figure 3-22. Conformational properties of aSyn fibrils amplified from PD, MSA and DLB brain extracts.

(a) Differences in the protonation levels in aSyn fibrils amplified from brain extracts (PD/green, MSA/purple, DLB/orange) when compared to those observed for the hsAsyn in vitro polymorph. Difference values were calculated on the basis of the average protonation levels observed for two patient samples each. The location of extended cross- $\beta$-structure within the hydrogenbonded core of the hsAsyn fibril is shown on top. NACore is the most hydrophobic part of the aSyn sequence. (b) Mapping of protonation level differences between PD and hsAsyn (green curve in (a)) on the core structure of hsAsyn fibrils (PDB code: 6A6B). Differences are color coded according to the scale bar and as illustrated in (a). Residues that experience faster solvent exchange in PD fibrils are shown in magenta/pink, those with slower solvent exchange in cyan. Residues with signal overlap were excluded from the analysis (shown in grey). (c,d) Protonation level differences between aSyn fibrils amplified from PD- and MSA-brain extracts, mapped in (d) on the core structure of hsAsyn fibrils. Residues that experience faster solvent exchange in MSA fibrils (when compared to PD fibrils) are shown in orange, those with slower solvent exchange in green. Residues with signal overlap were excluded from the analysis (shown in grey in (d)). 
a

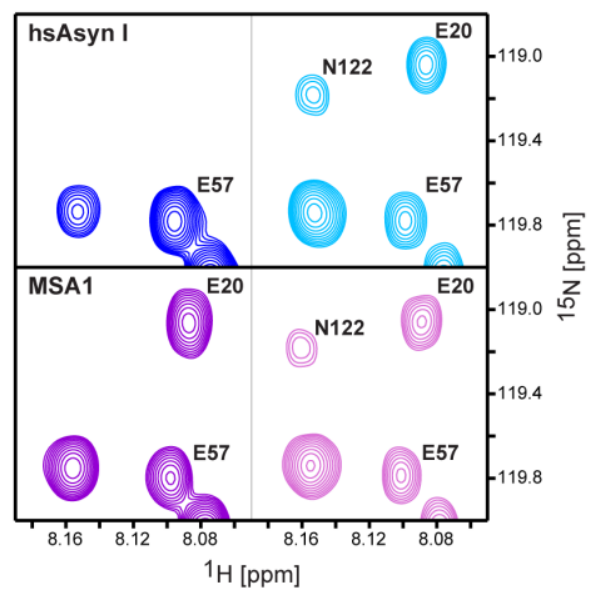

b


Figure 3-23. Extended data for brain-extract seeded fibrils.

(a) Selected region from ${ }^{1} \mathrm{H}-{ }^{15} \mathrm{~N}$ correlation spectra recorded after fibril dissociation at the beginning (left) and end (right) of the back-exchange period for hsAsyn I fibrils in blue in comparison to MSA1 fibrils in purple. (b) Comparison of HD exchange profiles of aSyn fibrils amplified from the brain extracts of three different PD patients. HD exchange profiles of PD1 and PD2 are the same shown in Figure 3-21. (c) Comparison of HD exchange profiles of aSyn fibrils, which were obtained through seeding with PMCA-amplified fibrils from the same DLB patient. 


\subsection{EPR spectroscopy of aSyn fibrils}

To further support structural differences between aSyn fibrils amplified from patient brain extracts and in vitro polymorphs, electron paramagnetic resonance (EPR) was used. In particular, to focus on the E83-G93 region (Figure 3-22 b). The recombinant aSyn-T54C/A90C mutant protein was labeled at the two cysteines with the nitroxide tag MTSL, and mixed with wild-type aSyn at a molar ratio of 1:30 (aSynT54C/A90C:aSyn) ${ }^{165}$. This mixture was subsequently aggregated into amyloid fibrils through seeding with either in vitro aSyn polymorphs or brain extract-amplified fibrils.

Continuous wave EPR spectra of all samples (hsAsyn, IsAsyn, PD, MSA, DLB) showed broad EPR lines (Figure 3-24 a,b), demonstrating that the MTSL-labeled aSyn-T54C/A90C protein was incorporated into fibrils ${ }^{176}$.

a

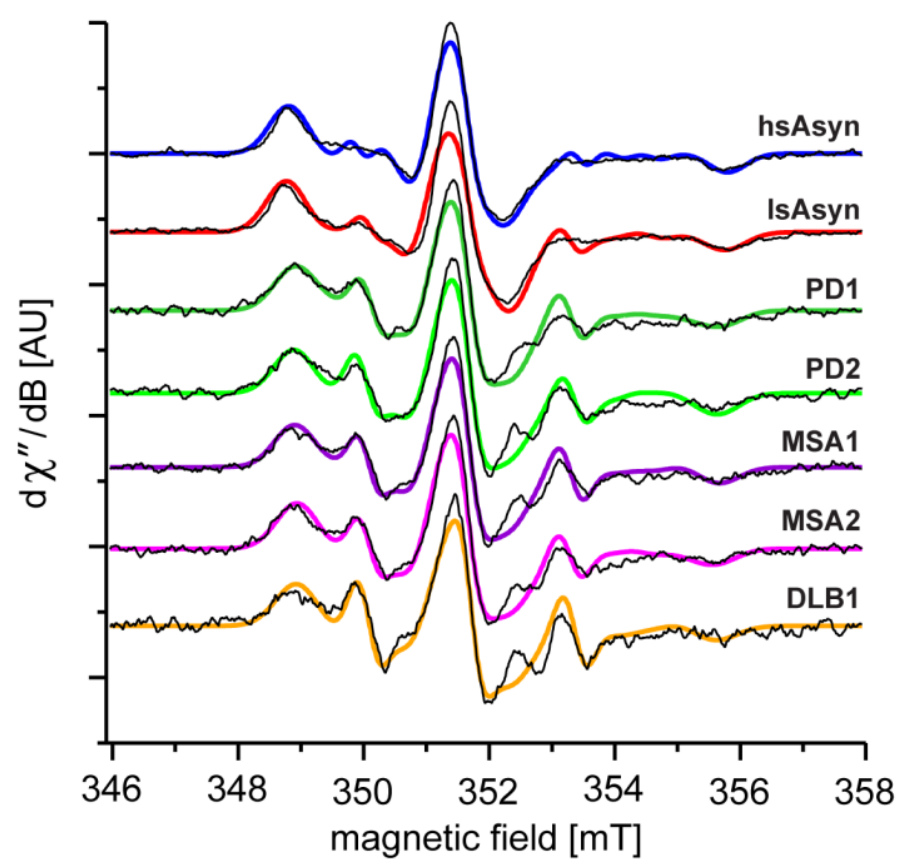

b

\begin{tabular}{|l|c|}
\hline \multicolumn{1}{|c|}{ sample } & \multicolumn{1}{c|}{$\begin{array}{r}\text { ratio } \\
\text { fast/slow }\end{array}$} \\
\hline hsAsyn & 0.01 \\
\hline IsAsyn & 0.01 \\
\hline PD1 & 0.06 \\
\hline PD2 & 0.06 \\
\hline MSA1 & 0.07 \\
\hline MSA2 & 0.06 \\
\hline DLB1 & 0.11 \\
\hline
\end{tabular}

Figure 3-24. Continuous wave EPR spectra of aSyn fibrils.

(a) Continuous wave EPR spectra of aSyn fibrils. Fits derived from a two-spin simulation are shown as colored lines. (b) Dynamic parameters (weight: fast/slow) of MTSL spins derived from fitting continuous wave EPR spectra shown in (a). For both spin species $g \approx[2.008,2.006$, 2.002] and $\mathrm{A} / \mathrm{h} \approx[17.8-19,18.5-19,93-110] \mathrm{MHz}$. The peak-to-peak isotropic linewidths (Iwpp) for the two species were $\operatorname{Iwpp}_{(\text {(low) }}=0.52-0.67 \mathrm{mT}$ and $\operatorname{lwpp}_{\text {(fast) }}=0.3-0.4 \mathrm{mT}$, respectively. 
a
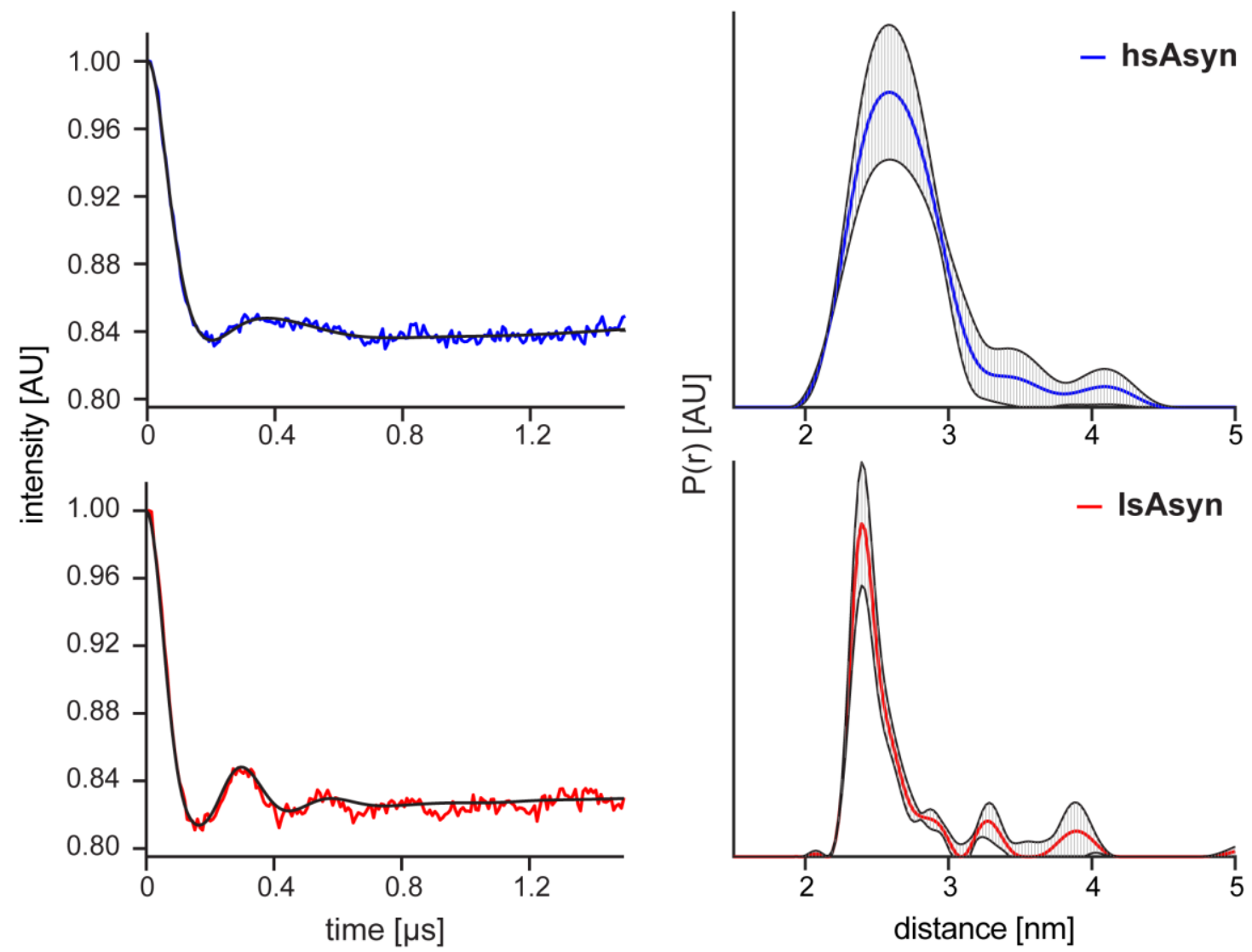

b
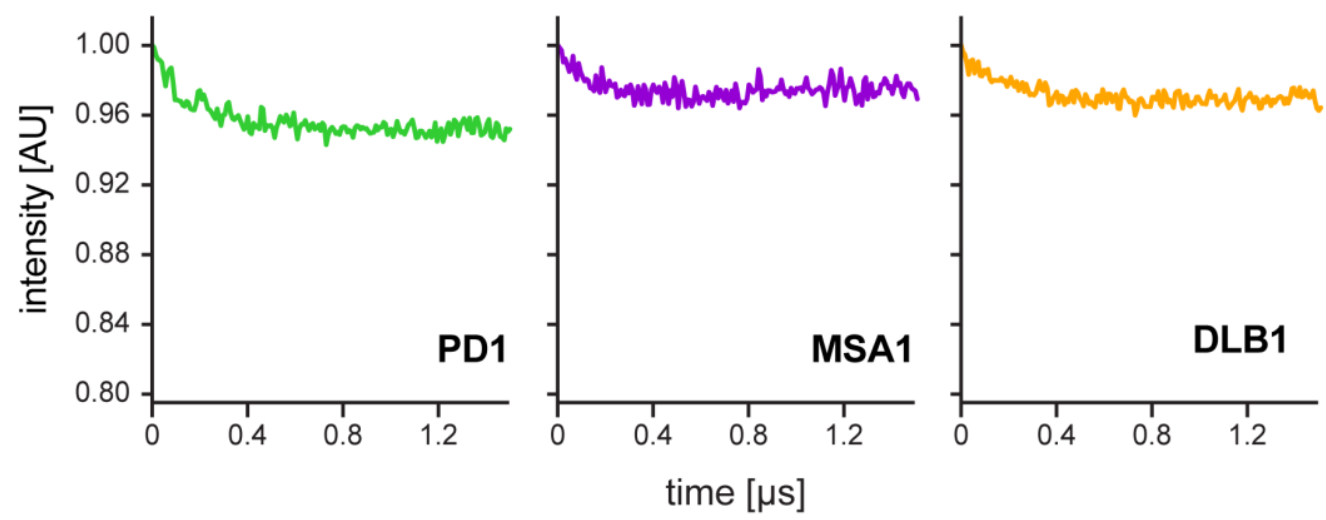

Figure 3-25. Dipolar modulation of aSyn fibrils.

(a) Dipolar modulation (a; corrected for background; fit in blue/red) and normalized distance distribution determined by $34 \mathrm{GHz}$ four-pulse PELDOR experiments for hsAsyn (top, blue) and IsAsyn (bottom, red). (b) Background-corrected PELDOR traces of aSyn fibrils amplified from PD, MSA and DLB brain extracts. Electron micrographs of the aSyn fibrils are shown in Figure 3-5. 
Pulsed EPR distance measurements determined the distance between the two MTSLlabels at position 54 and 90 of the hsAsyn polymorph to be $~ 2.4-2.6 \mathrm{~nm}$ (Figure 3-25 a), in agreement with the structure of hsAsyn (Figure 2-8 b) and previous EPR data ${ }^{165}$. A similar yet slightly shorter distance was determined for IsAsyn (Figure 3-25 a). In contrast, no pronounced dipolar modulation was observed for any of the aSyn fibrils amplified from the brain extracts (Figure 3-25 b and Figure 3-26), despite the identical experimental parameters and comparable signal-to-noise ratios (Figure 3-26). The lack of dipolar modulations for the T54C/A90C-interaction in aSyn fibrils amplified from brain extracts suggests that these two residues do not have a single, defined distance in these fibrils (Figure 3-5). This could be caused by a number of reasons (alone or combined), including higher flexibility of the MTSL side chains, increased flexibility of the backbone, and larger heterogeneity/polymorphism in aSyn fibril conformations in these samples. Consistent with increased backbone flexibility/polymorphism, protonation levels of A85-G93 were lower in aSyn fibrils amplified from brain extracts when compared to the two in vitro polymorphs (Figure 3-22 a,b). In addition, estimation of MTSL mobility on the basis of continuous wave EPR spectra suggested that at least one of the two MTSL labels was more flexible in aSyn fibrils amplified from brain extract when compared to aSyn in vitro polymorphs (Figure 3-24 a,b). Because HD-exchange studies showed that the cross- $\beta$-structure of residues A85-G93 is destabilized in fibrils amplified from patient brain extracts (Figure 3-21 b, Figure 3-24 a), the MTSL with higher flexibility could be located at position 90 .

a

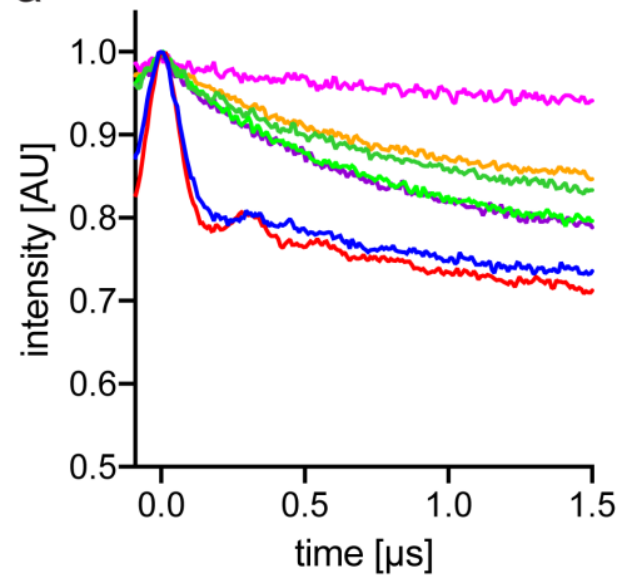

b

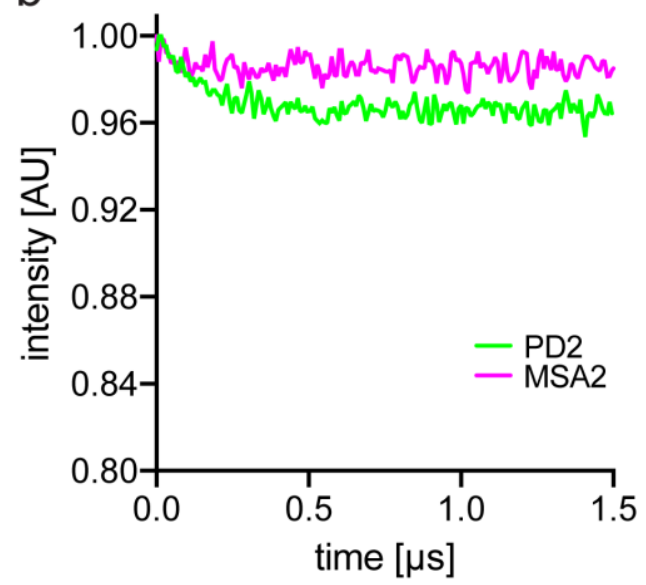

Figure 3-26. Dipolar modulation of aSyn fibrils, extended information.

(a) $34 \mathrm{GHz}$ four-pulse PELDOR traces (without background correction) of different aSyn fibrils.

(b) Background-corrected PELDOR traces of aSyn fibrils amplified from PD2 and MSA2 brain extracts. 


\section{Discussion}

\subsection{Evaluation HD exchange coupled to NMR spectroscopy}

HD exchange coupled to NMR spectroscopy previously identified distinct cross- $\beta$ sheet cores in two prion strains ${ }^{177}$. A strength of the HD exchange method is that all molecules contribute to the NMR-observed protonation levels, which therefore characterize the full spectrum of fibril conformations present in each sample. This is important, because aSyn fibrils purified from patient brains are often polymorphic ${ }^{4,54}$.

\subsubsection{Accessibility of structural polymorphism by HD exchange}

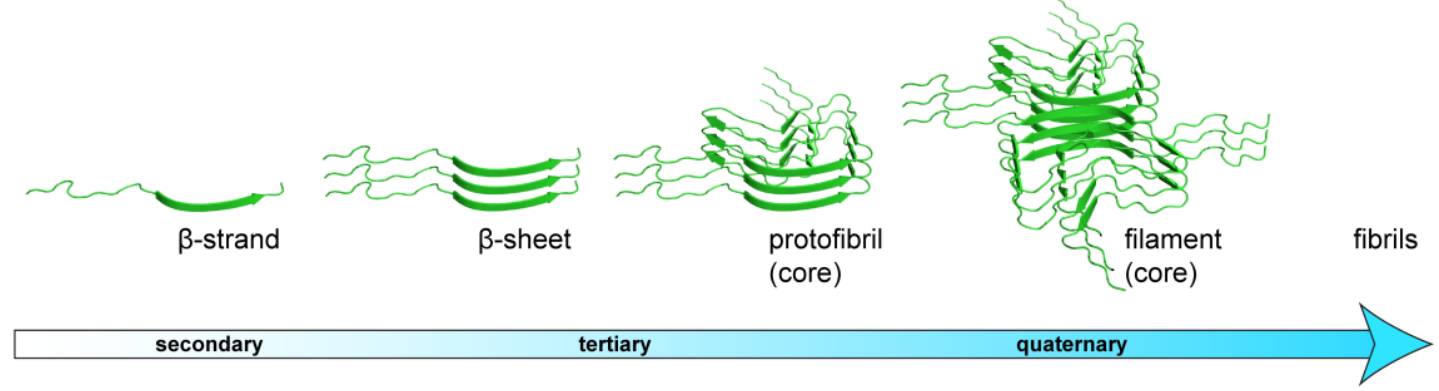

Figure 4-1 Structural complexity of aSyn fibrils.

High salt synuclein cryo-EM structure was used (PDB code: 6A6B). Increase in structural complexity from left to right indicated by arrow. The terms "secondary", "tertiary" and "quaternary" do not strictly follow the conventions of globular proteins, see Fitzpatrick et al. ${ }^{178}$.

All levels of the structural complexity of aSyn fibrils (Figure 4-1) potentially influence hydrogen-bonding networks and solvent accessibility of the amide backbone. Inregister intermolecular $\beta$-sheets strongly protect amide protons from hydrogendeuterium forward exchange, as well as the tertiary architecture of the aSyn monomer perpendicular to the fibril axis. Quaternary effects such as protofibril interfaces may also influence hydrogen-deuterium forward exchange. HD exchange coupled to NMR spectroscopy also provides information about more flexible regions in amyloid fibrils. In contrast, aSyn fibril structures obtained by cryo-electron microscopy ${ }^{134,135}$ are limited to the highly ordered fibrillar core region, e.g. residues V37-Q99 (PDB code: 6A6B) and L38-V95 (PDB code: 6H6B) respectively.

As shown in Figure 3-21 b, aSyn fibrils seeded by brain extract of MSA showed consistently higher protonation levels within residues A18-V48 than fibrils obtained from PD patients, with DLB aSyn fibrils lying in between. Taking into consideration a much stronger effect for the protonation level of the $\sim 30$ residue $\mathrm{N}$-terminus of IsAsyn 
in vitro fibrils (Figure 3-19 a) with respect to the hsAsyn polymorph, aSyn fibril polymorphism among brain extract seeded aSyn fibrils may be due to extension of the central fibrillar core region. Specially for MSA brain extract-seeded fibrils it is not clear, if the higher protonation level from A18-V48 reflect a pure fibril conformation or the effect is due to a superimposition of aSyn fibrils showing high protonation levels and aSyn fibrils of lower protonation levels within the same region.

HD exchange coupled to NMR spectroscopy is an orthogonal method to highresolution cryo-electron microscopy as shown for the mapping of hsAsyn fibril protonation levels to the fibrillar core of the cryo-EM structure of hsAsyn fibrils (Figure 3-17 a). However, in the absence of high molecular cryo-EM structures for aSyn fibrils seeded by brain-extracts, the interpretation of the observed protonation levels on the tertiary and quaternary structural level remains partially speculative. Nevertheless, the mapping of the protonation level differences to the hsAsyn polymorph for aSyn fibrils seeded by PD patients' brain, clearly identified sequential patches within the protofibril core that differ, hinting at conformational differences in particular for residues A85-G93 (Figure 3-22 a,b). In addition, changes in the protonation levels for residues A53-A56 in case of MSA- and DLB-seeded aSyn fibrils might reflect changes in quaternary structure (Figure 3-22 c,d).

\subsubsection{Protonation levels in aSyn fibrils seeded by brain extracts}

Li et al. ${ }^{173}$ have recently solved the cryo-EM structure of two main conformations for the aggregation product of in vitro aggregated full-length aSyn fibrils, the "rod" and "twister" polymorph. They suggested a model (Figure 4-2) where the protofibril interface determines the fibrillar core structure. This model would explain why aSyn fibrils seeded from brain extract of MSA - and to a smaller extent also DLB-seeded aSyn fibrils - differ from aSyn fibrils of PD patients in the interface as well as the protonation level within the C-terminus of the fibrillar core.

As noted for aSyn fibrils seeded by brain extracts of MSA, an additional level of structural variability/heterogeneity occurs in regions proximal to the central core region. Proteinase-K digestion of aSyn fibrils purified from the brain of an individual pathologically confirmed with MSA resulted in three major bands migrating at 7,8 and $9 \mathrm{kDa}$ with the 7 and $8 \mathrm{kDa}$ bands starting at $\mathrm{G} 31^{179}$. The $\mathrm{N}$-terminus of the $9 \mathrm{kDa}$ band was mapped to $A 19 / E 20^{179}$, in agreement with the finding that the protonation levels of residues A19-A29 exceed $50 \%$ in aSyn fibrils amplified from MSA brain extracts (Figure 3-21 b). 


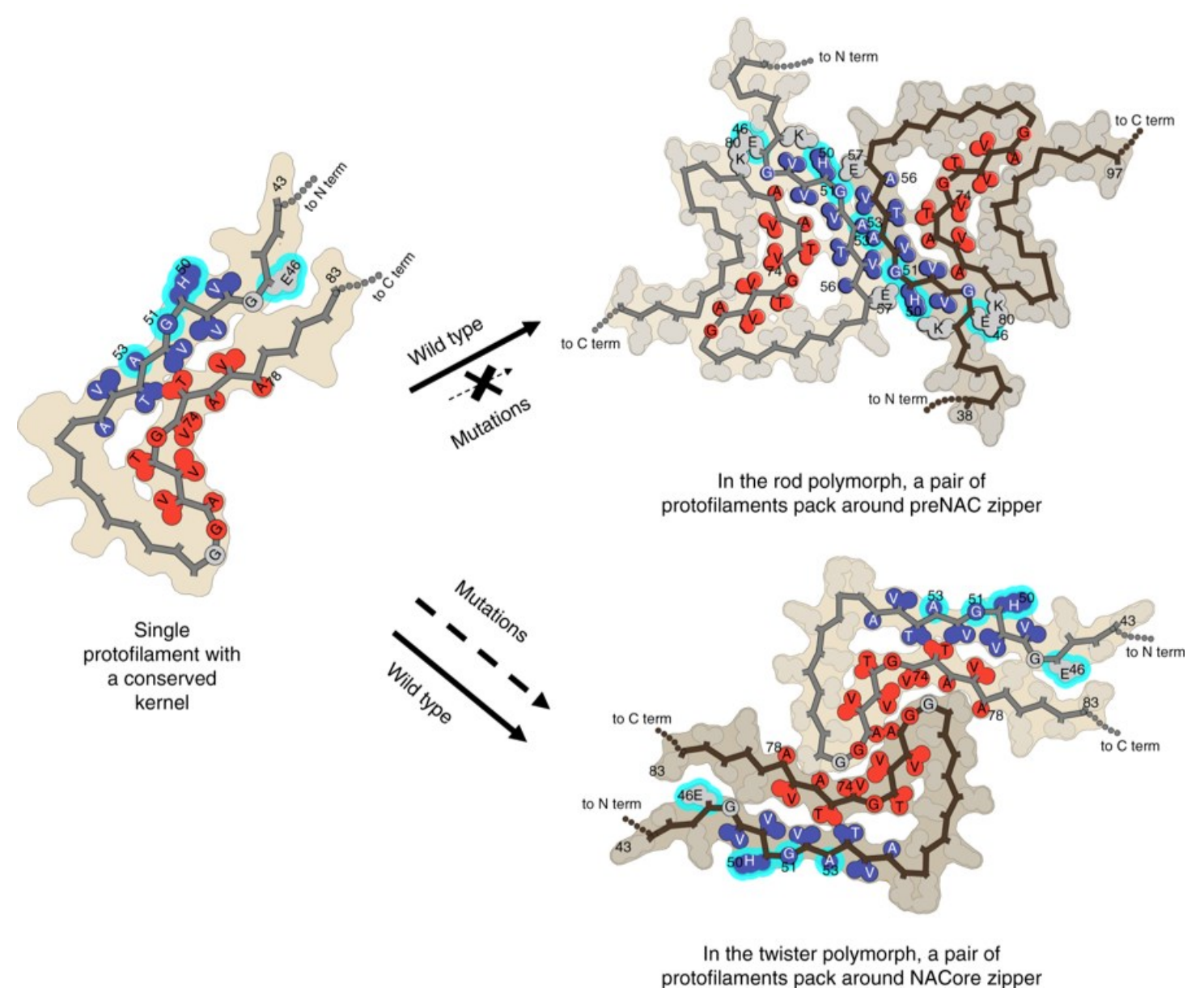

Figure 4-2. Different packing arrangements of the same protofilament kernel lead to polymorphic structures.

Model suggesting that protofilaments sharing a generic $\beta$-arch conformation (left) can assemble into aSyn filaments of different polymorphic structures, by forming distinct protofilament interfaces. The rod fibril polymorph forms the protofilament interface via the preNAC sequence (upper right), the twister fibril polymorph through the NACcore region (bottom right). The figure was taken from Li et al. ${ }^{173}$.

\subsubsection{Analysis of a broad range of aSyn fibrils}

The comparison of aSyn fibrils seeded by brain extract of several patients from three different synucleinopathies (PD, MSA and DLB) in combination with the characterization of two in vitro polymorphs, hsAsyn and IsAsyn, presented a major challenge. Including the control experiments a high number of aSyn fibrils had to be structurally characterized. I therefore chose a unique approach of combining several techniques applied in the field for structural elucidation of amyloid fibrils.

The chosen strategy centered on the residue-specific determination of protonation levels by HD exchange coupled to NMR spectroscopy. A particular 
strength of HD exchange coupled to NMR spectroscopy is the feasibility to study a larger number of fibrils in a justifiable amount of time. Transmission electron microscopy (TEM) was very helpful in addressing questions about the general morphology of the fibrils. The fibrillar core diameter observed by TEM already permitted important insights in quaternary structure of amyloid fibrils, reporting the number of protofibrils $(\sim 5 \mathrm{~nm})$ the amplified fibrils contained. Circular dichroism allowed a qualitative read-out of the fibril aggregation assay, the formation of cross- $\beta$ aggregates and the consumption of intrinsically disordered aSyn monomers.

With the fluorescence dyes, established molecular probes were used to look into the fine structure of aSyn fibrils. The combination of curcumin, FSB and HS-68 not only led to clear distinction of in vitro polymorphs from brain-extract seeded aSyn fibrils, it also hinted at more subtle differences among the different synucleinopathies (PD, MSA and DLB).

Electron paramagnetic resonance on MTSL-labeled aSyn mutant (aSyn T54C/A90C) incorporated into in vitro aSyn polymorphs yielded distance constraints for the tertiary protofibril architecture of aSyn. In addition, continuous wave (CW) EPR spectra provided information about the flexibility of the MTSL-labels and acted as atomic probes of the local environment of the MTSL-label within the aSyn fibrils. The absence of dipolar modulation of EPR was important for cross-validation of the HD exchange data. The broad EPR lines in CW spectra also directly reported on MTSLlabeled aSyn T54C/A90C mutant incorporation into fibrils, for both in vitro aSyn polymorphs as well as brain-extract seeded aSyn fibrils ${ }^{165}$.

A structural characterization of the different amyloid fibrils by cryo-electron microscopy ${ }^{134}$ or solid-state NMR spectroscopy ${ }^{133,135}$ will be the target of future studies.

\subsection{Amyloid polymorphism in other neurodegenerative diseases}

\subsubsection{Alzheimer's disease}

In Alzheimer's disease (AD) two distinct morphologies of neurofibrillary tangles (NFTs) of tau are found in AD patients, paired helical filaments (PHFs) and straight filaments (SFs) ${ }^{180}$. Fitzpatrick et al. ${ }^{181}$ solved the cryo-EM structure for the fibrillar core of the two tau polymorphs directly isolated from an AD patient's brain. The study showed that paired helical and straight filaments differ in their protofibril interface with the cores formed mainly by residues V306-F378 of R3 and R4 repeat of tau (Figure 4-3 a,b). 
a

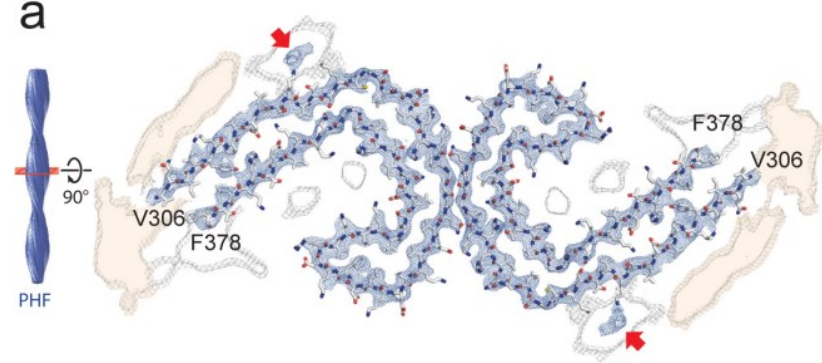

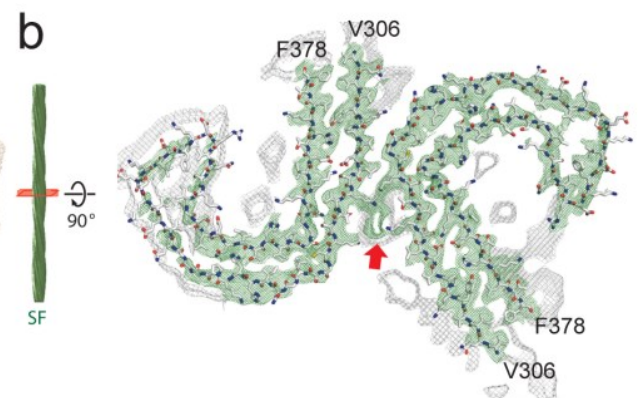

Figure 4-3. Cross-sections of the PHF and SF cryo-EM structures.

Atomic models of (a) PHF and (b) SFs overlaid with cryo-EM density. High resolution maps are shown in blue (PHFs) and green (SFs), additional high-resolution electron density that could not be assigned is indicated by red arrows. Lower resolution electron density is shown in grey, for PHF electron density in orange represents less ordered $\beta$-sheet accommodating 16 additional amino acids of potentially R1 and/or R2 tau repeats. Taken from Fitzpatrick et al. ${ }^{181}$.

The work by Fitzpatrick et al. is currently the strongest evidence for structural polymorphs in tauopathies. A particular strength of this work is the structural elucidation of NTFs directly isolated from a single patient's brains, thus not requiring any amplification step. The scheme proposed by Li et al. ${ }^{173}$ for two aSyn fibril in vitro polymorphs (Figure 4-2) that the protofibril interface determines the fibril packing and drives polymorphism seems to hold also true for NTFs of tau. Both types of tau NTFs, PHFs and SFs, occur in parallel within neuronal cells of a patient affected by AD. There is lack of information if upon propagation the structural information of PHFs and SFs is conserved. This would be required to support a true prion-like behavior ${ }^{182}$. In addition, Fitzpatrick et al. ${ }^{181}$ conducted their research on a single AD patient, intersubject variability could therefore not be assed. Because more than 20 neurodegenerative disorders are linked to tau inclusions ${ }^{183}$, structural tau polymorphism might be an underlying disease-defining phenomenon.

\subsubsection{Tau inclusions in Pick's disease}

Pick's disease (PiD) is associated with tau inclusions formed by the human $3 R$ tau isoform lacking R2 repeat (Figure 4-4 a). Two polymorphs were observed in patients, the narrow pick filament (NPF) and wide pick filament (WPF). The wide pick filament consists of two NPFs, shown by Falcon et al. ${ }^{184}$. This is different from AD, where both SFs and PHFs consist of two protofibrils with distinct interfaces and packing (Figure 4-3). 
a

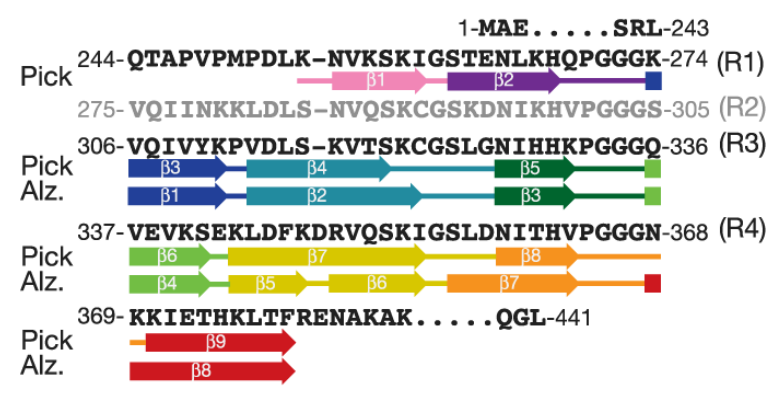

b

Pick fold
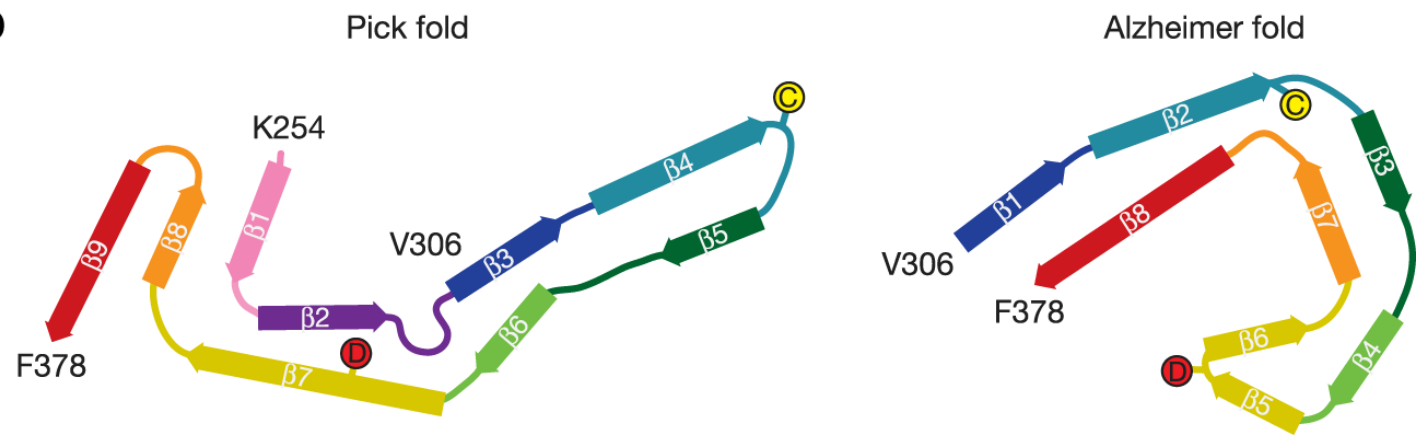

Figure 4-4. Polymorphic structure of tau inclusions in AD and PiD in comparison.

(a) Sequence alignment of the $\beta$-strands present in tau inclusions of Alzheimer's disease (Alz.) and Pick's disease (Pick). R2 repeat is missing in $3 R$ tau isoform for PiD, numbering follows hTau40. (b) Pick fold of NPF on the left, protofibril of SFs and PHFs of AD on the right from Fitzpatrick et al. ${ }^{181}$. Figure was taken from Falcon et al. ${ }^{184}$.

In addition to the quaternary differences, the tau protofibril fold in Pick's disease is distinct from Alzheimer's disease (Figure 4-4 b). Notable is the absence of a triangular arrangement of $\beta$-strands in the Pick fold formed by residues from K343 to I354, named $\beta 6$ and $\beta 7$ in the Pick fold (corresponding to $\beta 4-6$ for the AD fold). The triangular arrangement is similar to $\beta$-helices, that were associated with amyloid fibrils of prion disease $^{185}$. Another distinct feature of the Pick fold is the $\beta$-hairpin following C322 between $\beta 4$ and $\beta 5$, which is not observed for the Alzheimer fold. The interaction of $\beta 7$ and $\beta 3$ of R3 tau repeat in AD occurs in a similar way for $\beta 8$ and $\beta$ of R1 in Pick's disease.

The fibrillar core of the AD fold is comprised by R3 and R4, whereas R2 and $\mathrm{R} 1$ are part of the fuzzy coat. The Pick's fold, that is only associated with the $3 R$ tau isoform shows a very distinct fold architecture in the absence of R2, most strikingly R1 is part of the fibrillar core, not observed in Alzheimer's disease. This led to the hypothesis, that tau might also be able to show disease-specific fibrillar core folds in 
tau pathologies associated with the same $4 \mathrm{R}$ tau isoform composition as in $A D^{184}$ such as for example progressive supranuclear palsy.

4.2.3 Disease-associated amyloid polymorphism in tauopathies and synucleinopathies

The high resolution cryo-EM structures of ordered fibril cores in Alzheimer's disease (4R tau), both paired helical and straight filaments, and the fibrillar core fold of Pick's disease (3R tau) together show strong evidence for structural polymorphism in tauopathies. Polymorphism on the level of changes between the tertiary structure of the protofibril fold, AD fold versus Pick fold, as well as quaternary changes in the packing with different protofibril interfaces, as observed for PHFs vs SFs in Alzheimer's disease. To finally evaluate the disease-specific role of structural polymorphism in tauopathies, the same tau isoform ( $4 R$ and/or $3 R$ ) has to be observed in different conformations for at least two distinct neurodegenerative diseases associated with tau aggregates.

The current state of the field does not sufficiently address the "fuzzy coat" of tau fibrils, comprising R1 entirely and most likely also parts of R2 for Alzheimer's disease and the N-terminus of R1 in Pick's disease. On top of that for PiD as well as $A D$, the core region of tau aggregates extends in the R' region of tau, extending beyond the R4 tau repeat (Figure 4-4 a).

In the current study on aSyn fibrils amplified from patient brain material, differences in the protonation levels were observed in a disease-specific manner for residues preceding the fibrillar core region, which was described by cryo-EM for in vitro aSyn fibrils. In case of aSyn fibrils amplified from brain material of a patient pathologically confirmed with MSA, residues A18 to V48 showed slower amide proton exchange when compared to DLB- and PD-seeded fibrils. The aSyn fibrils seeded by PD brain extract, vary also from MSA-seeded and to a lesser extent from DLB-seeded aSyn fibrils in the C-terminus of the core region from E83 to K96. Similar to tau aggregates, the aSyn fibril core-flanking regions and the absolute core extension itself might differ disease-specifically.

In the field of synucleinopathies, there are so far no high-resolution structures of fibrils isolated from patient brains available. In that sense, tau aggregates are much better characterized. This might have to do with the higher abundance of tau aggregates in the brain cortex compared to aSyn fibrils in synucleinopathies. On the other hand, the disease-specificity and strain-like behavior of aSyn pathology in disease progression models is better characterized in the $\alpha$-synuclein field due to the 
studies of Prusiner et al. ${ }^{124}$, Bernis et al. ${ }^{125}$ and Peng et al. ${ }^{186}$. In addition, direct spread of amyloid pathology from host-to-grafted tissue in patients, as shown for Lewy body pathology by Kordower et al. ${ }^{114}$ and Li et al. ${ }^{115}$, have so far not been reported for tauopathies.

The amplification of aSyn fibrils seeded by brain-extracts of patients, as achieved in the current work by PMCA, introduces an additional level of complexity when compared to amyloid fibrils purified from patient brain and characterized by cryoEM. However, amplification of aSyn fibrils by PMCA is connected to and the resulting structural polymorphism observed follows strictly the prion-like definition, implying that structural differences between amyloid fibrils must be maintained during seeded protein aggregation.

\subsubsection{Structural characterization of $A \beta$ aggregates from $A D$ patient brain material}

The amyloid precursor protein (APP) derived peptides amyloid- $\beta 40$ (A $\beta 40)$ and amyloid- $\beta 42$ (A $\beta 42)$ are the most common cleavage products obtained by enzymatic proteolytic cleavage of APP trough $\alpha-, \beta$ - and $\gamma$-secretases ${ }^{187}$. Both isoforms form extracellular amyloid plaques in Alzheimer's disease with $A \beta 42$ being the most aggregation prone isoform in vitro ${ }^{188}$.

For $\mathrm{A} \beta 40$, two distinct in vitro polymorphs ${ }^{189,190,191}$ were observed by solid-state NMR spectroscopy under the same buffer condition, protein concentration, ionic strength, $\mathrm{pH}$ and temperature (Figure 4-5 b,c). The "striated ribbon" polymorph was obtained by either de novo aggregation of $A \beta 40$ under gentle agitation or seeded amplification using aforementioned de novo aggregated $A \beta 40$ fibrils as seeds ${ }^{190}$. In the absence of agitation under quiescent conditions, $A \beta 40$ formed a second polymorph that could also be amplified by seeded aggregation called "twisted ribbon", reflecting the twisted morphology in negative-stain transmission electron microscopy. Paravastu et al. ${ }^{191}$ stated, that with this approach a polymorph homogeneity of in vitro $A \beta$ fibrils higher than $90 \%$ was achieved for both polymorphs. The two polymorphs differ in their quaternary protofibril interfaces and the overall symmetry, the "striated ribbon" is formed by two $A \beta 40$ units (Figure $4-5$ b), the "twisted ribbon" shows a threefold symmetry formed by three $A \beta 40$ units (Figure $4-5 \mathrm{c}$ ). The tertiary structure is highly conserved and both in vitro polymorphs show parallel in-register $\beta$-sheets. Both structural models start at residue G9, suggesting a flexible $\mathrm{N}$-terminus. In addition to the "striated" and "twisted" in vitro polymorph of $A \beta 40$, there exists another low resolution in vitro polymorph cryo-EM model for de novo aggregated $A \beta 40$ fibrils 
determined by Sachse et al. ${ }^{192}$, comprised of two protofibrils with yet another suggested protofibril interface distinct from the "striated" and "twisted" polymorph. For the two in vitro polymorphs of $A \beta 40$, variations in toxicity were observed in cell culture of primary neurons ${ }^{189}$ which might indicate a potential prion-like behavior.

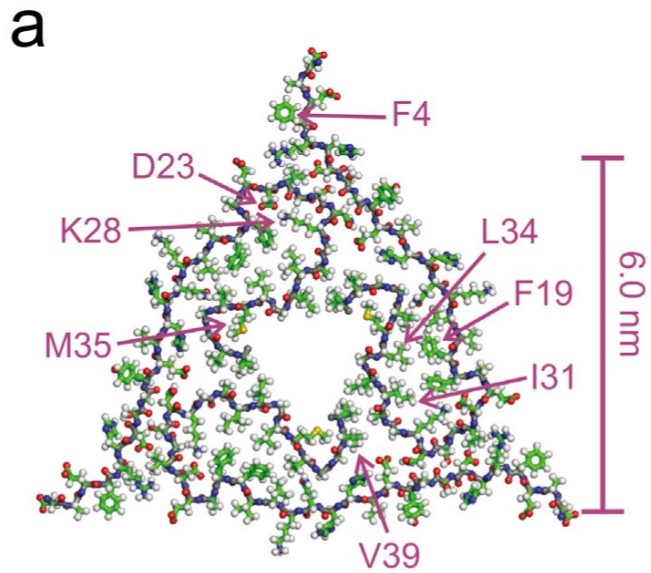

$\mathrm{b}$

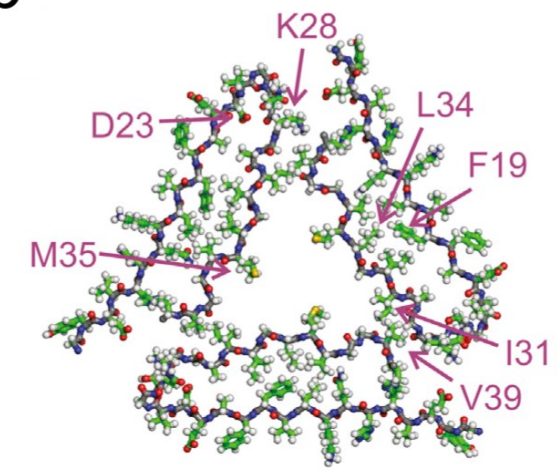

C



Figure 4-5. A $\beta 40$ solid-state NMR structures.

(a) A 340 seeded by AD patient material (patient I). (b) In vitro aggregated A 340 fibrils, "twisted ribbon polymorph". (c) "Striated ribbon" polymorph obtained through in vitro aggregated of A $\beta 40$. Figure taken from Lu et al. ${ }^{193}$.

A $\beta 40$ fibrils seeded by an aggregate-enriched fraction of brain extract from two $A D$ patients were characterized and for a single patient (patient $\mathrm{I}$ ) a high-resolution solid-state NMR structure was obtained (Figure 4-5 a) ${ }^{193}$. Upon seeding of $A \beta 40$ with $A D$ patient-derived material, $A \beta 40$ fibrils of high homogeneity were obtained. The $A \beta 40$ fibril seeded by aggregate-enriched brain fractions of patient I showed threefold symmetry similar to the "twisted ribbon" in vitro A $\beta 40$ polymorph, but differs from both in vitro polymorphs, twisted and striated ribbon, in the protofibril tertiary fold. In contrast to the in vitro polymorphs, the N-terminus formed part of the cross- $\beta$ fibrillar core of brain-extract amplified A $\beta 40$ fibrils. Notably, no high-resolution model was determined 
for the second patient-derived $A \beta 40$ fibrils, which were suggested to differ from those of patient I.

In case of $A \beta 42$, high-resolution structures of amyloid fibrils, which were in vitro aggregated in different conditions, were determined. Two earlier studies reported different structural models, one obtained by cryo-EM for de novo aggregated in vitro fibrils at $5 \AA$ resolution ${ }^{194}$, the other one derived by solid-state NMR spectroscopy ${ }^{195}$. The cryo-EM model of the in vitro aggregated $A \beta 42$ fibrils showed two-fold symmetry and consisted of two protofibrils reminiscent of the cryo-EM structure of $A \beta 40$ in vitro fibrils $^{192}$. The solid-state NMR structure of $A \beta 42$ fibrils was obtained by a sequential repetitive seeding approach to amplify the dominant fibril polymorph. The structure revealed a single protofibril formed by three $\beta$-strands forming a "triple $\beta$-motif" starting at E11. Two independent research groups subsequently published highly similar refined structural models of in vitro A $\beta 42$ fibrils also obtained by solid-state NMR spectroscopy ${ }^{196,197}$ that confirmed the protofibril fold first described by Xiao et al. ${ }^{195}$. Differences in the tertiary protofibril fold between Xiao et al.'s model and the two more recent studies ${ }^{196,197}$ are due to the newer studies being more refined with a higher number of intramolecular restraints. Wälti et al. ${ }^{196}$ and Colvin et al. ${ }^{197}$ independently suggested an identical protofibril interface for $A \beta 42$ in vitro fibrils with two-fold symmetry. The intermolecular protofibril contacts are based on a number of intermolecular restraints. The hydrophobic residues L34, M35 and V36 form intermolecular contacts with V18, L17, K16 and Q15 and define the protofibril interface. For both dimeric solid-state structures, the $\mathrm{N}$-terminus is highly flexible and not involved in cross- $\beta$ fibril core. In a cryo-electron study of $A \beta 42$ fibrils obtained by in vitro aggregation under quiescent conditions, a different fibril quaternary architecture was found ${ }^{198}$. The protofibril interface was also formed by a dimer, however the intermolecular contacts are mainly due to interactions of the $\mathrm{C}$-terminus with the $\mathrm{C}$ terminus of the adjacent protofibril, showing a unique quaternary fibril architecture. Strong differences were also observed on the tertiary level of the $A \beta 42$ protofibril fold. Though the cryo-EM structure by Gremer et al. ${ }^{198}$ is in agreement with the C-terminus of the solid-state NMR structures from residue S26 to A42, the N-terminus in contrast strongly deviates and forms part of the protofibril core.

Qiang et al. ${ }^{199}$ compared $A \beta 40$ fibrils obtained by seeding with patient material of $A D$ patients diagnosed with distinct clinical disease subtypes and suggested the presence of a single dominant $A \beta 40$ fibril structure. This however might be due to the chosen amplification protocol and does not rule out the existence of distinct diseaserelevant polymorphs in vivo. In addition, Qiang et al. ${ }^{199}$ seeded A $\beta 42$ peptides by brain 
material from patients diagnosed with clinical subtypes of $A D$ and identified two distinct fibril conformations using 2D solid-state NMR.

The currently available structures for $A \beta 40$ and $A \beta 42$ support the importance of structural polymorphism. The solid-state NMR structures of $A \beta 40$ (Figure 4-5) are consistent and follow the observations for aSyn and tau, that the flanking regions of the fibrillar core are a source of polymorphism and that polymorphism occurs in the packing with distinct protofibril interfaces and quaternary cross- $\beta$ folds. 


\section{Conclusions and perspectives}

This study establishes a strong correlation between aSyn structure and disease phenotype. The disease-specific polymorphs characterized in this study are distinct from in vitro amplified aSyn fibrils obtained by seeded aggregation under high and low salt conditions, shown in detail by HD exchange coupled to NMR spectroscopy, EPR spectroscopy and fluorescent dye binding. The data suggest, that the high-resolution structures of in vitro aSyn fibrils available in the field of synucleinopathies may not fully reflect structural features of disease-relevant polymorphs and differences on the quaternary and tertiary structural level are likely.

Even more importantly, the patient's disease classification - Parkinson's disease (PD), multiple system atrophy (MSA) or dementia with Lewy body (DLB) - is reflected in the polymorphic structure of aSyn fibrils seeded by brain extracts of the individual patients (Table 3 ) shown by hydrogen-deuterium (HD) exchange coupled to NMR spectroscopy. This observation proofs that the seeds present in brain extracts of patients not only catalyze the misfolding and aggregation of recombinant aSyn monomer but imprint structural features by templated seeding in a prion-like manner. Disease-relevant prion-like behavior of aSyn strains was already observed earlier in glial cytoplasmatic inclusions ( $\mathrm{GCl}$ ) with respect to aSyn aggregates of Lewy body (LB) pathology ${ }^{124,186}$. This is the first study however, that shows structural polymorphism between distinct synucleinopathies on the level of residue-specific conformations of aSyn fibrils and simultaneously correlates the finding to available high-resolution structures and known structural features of genuine in vitro aSyn polymorphs in the field. Along the way, it was also confirmed that protein misfolding cyclic amplification ${ }^{106}$ (PMCA) conserved key and disease-defining conformational properties of the initial patient-derived seeds characterized in this study at so far highest structural precision.

The implications of the data presented here are far-reaching. On one hand a further in-depth structural analysis of aSyn fibrils seeded by brain-extract of patients has to follow in subsequent studies to asses differences in the protofibril fold and quaternary aSyn structure, such as the fibrillar core symmetry and the exact protofibril interface. Cryo-electron microscopy as well as solid-state NMR spectroscopy are promising choices. On the other hand, it would be very interesting to know if the obtained disease-specific aSyn fibril polymorphs show also distinct toxicities in cell culture and animal models. This would be an important corner stone to shed further light on prion-like disease transmission and infectiousness of aSyn aggregates in synucleinopathies. 
The approach might also be applicable to neurodegenerative diseases caused by other proteins that undergo misfolding and aggregation, such as e.g. tau and $A \beta$. Specially for tau the existence of in vivo tau polymorphs is very well established for Alzheimer's disease and Pick's disease, but the propagation of structural conformations through seeded aggregation has not been sufficiently shown yet.

The disease-specific structural conformations of aSyn fibrils seeded by brainextracts of PD, MSA and DLB might open new targets for pharmaceutical research. This study is thus an important milestone in the understanding of aSyn pathology and contributes to the development of appropriate diagnostic and therapeutic agents in distinct synucleinopathies. 
6 Bibliography

1 Abbott, A. Dementia: A problem for our age. Nature 475, S2, (2011).

2 Sipe, J. D. \& Cohen, A. S. Review: history of the amyloid fibril. J Struct Biol 130, 88-98, (2000).

3 Parkinson, J. An essay on the shaking palsy. 1817. J Neuropsychiatry Clin Neurosci 14, 223-236; discussion 222, (2002).

4 Spillantini, M. G., Crowther, R. A., Jakes, R., Hasegawa, M. \& Goedert, $M$. alpha-Synuclein in filamentous inclusions of Lewy bodies from Parkinson's disease and dementia with lewy bodies. Proc Natl Acad Sci U S A 95, 6469-6473, (1998).

5 Alzheimer, A., Stelzmann, R. A., Schnitzlein, H. N. \& Murtagh, F. R. An English translation of Alzheimer's 1907 paper, "Uber eine eigenartige Erkankung der Hirnrinde". Clin Anat 8, 429-431, (1995).

6 Hardy, J. \& Allsop, D. Amyloid deposition as the central event in the aetiology of Alzheimer's disease. Trends Pharmacol Sci 12, 383-388, (1991).

7 Morishima-Kawashima, M. et al. Hyperphosphorylation of tau in PHF. Neurobiol Aging 16, 365-371; discussion 371-380, (1995).

8 Rowland, L. P. How amyotrophic lateral sclerosis got its name: The clinical-pathologic genius of jean-martin charcot. Archives of Neurology 58, 512-515, (2001).

9 Hardiman, O. et al. Amyotrophic lateral sclerosis. Nat Rev Dis Primers 3, 17071, (2017).

10 Rosen, D. R. et al. Mutations in Cu/Zn superoxide dismutase gene are associated with familial amyotrophic lateral sclerosis. Nature 362, 59-62, (1993).

11 George Huntington. On Chorea. The Journal of Neuropsychiatry and Clinical Neurosciences 15, 109-112, (2003).

12 MacDonald, M. E. et al. A novel gene containing a trinucleotide repeat that is expanded and unstable on Huntington's disease chromosomes. Cell 72, 971-983, (1993).

13 Katscher, F. It's Jakob's disease, not Creutzfeldt's. Nature 393, 11, (1998).

14 Prusiner, S. B. Novel proteinaceous infectious particles cause scrapie. Science 216, 136-144, (1982).

15 Walker, L. C. Proteopathic Strains and the Heterogeneity of Neurodegenerative Diseases. Annu Rev Genet 50, 329-346, (2016).

16 Wood, L. B., Winslow, A. R. \& Strasser, S. D. Systems biology of neurodegenerative diseases. Integr Biol (Camb) 7, 758-775, (2015).

17 Wu, Y. T. et al. Dementia in western Europe: epidemiological evidence and implications for policy making. Lancet Neurol 15, 116-124, (2016).

18 Shibasaki, Y., Baillie, D. A., St Clair, D. \& Brookes, A. J. High-resolution mapping of SNCA encoding alpha-synuclein, the non-A beta component of Alzheimer's disease amyloid precursor, to human chromosome $4 q 21.3-->q 22$ by fluorescence in situ hybridization. Cytogenet Cell Genet 71, 54-55, (1995). 
19 Maroteaux, L., Campanelli, J. T. \& Scheller, R. H. Synuclein: a neuronspecific protein localized to the nucleus and presynaptic nerve terminal. J Neurosci 8, 2804-2815, (1988).

20 Nakajo, S. et al. Purification and characterization of a novel brainspecific 14-kDa protein. J Neurochem 55, 2031-2038, (1990).

21 Nakajo, S., Tsukada, K., Omata, K., Nakamura, Y. \& Nakaya, K. A new brain-specific 14-kDa protein is a phosphoprotein. Its complete amino acid sequence and evidence for phosphorylation. Eur J Biochem 217, 1057-1063, (1993).

22 Ueda, K. et al. Molecular cloning of cDNA encoding an unrecognized component of amyloid in Alzheimer disease. Proc Natl Acad Sci U S A 90, 11282-11286, (1993).

23 Jakes, R., Spillantini, M. G. \& Goedert, M. Identification of two distinct synucleins from human brain. FEBS Lett 345, 27-32, (1994).

$24 \mathrm{Ji}, \mathrm{H}$. et al. Identification of a breast cancer-specific gene, BCSG1, by direct differential cDNA sequencing. Cancer Res 57, 759-764, (1997).

25 Ohtake, H. et al. Beta-synuclein gene alterations in dementia with Lewy bodies. Neurology 63, 805-811, (2004).

26 Fujita, M. et al. A beta-synuclein mutation linked to dementia produces neurodegeneration when expressed in mouse brain. Nat Commun 1, 110, (2010).

27 Eliezer, D., Kutluay, E., Bussell, R., Jr. \& Browne, G. Conformational properties of alpha-synuclein in its free and lipid-associated states. $\mathrm{J} \mathrm{Mol}$ Biol 307, 1061-1073, (2001).

28 Fauvet, B. et al. alpha-Synuclein in central nervous system and from erythrocytes, mammalian cells, and Escherichia coli exists predominantly as disordered monomer. J Biol Chem 287, 15345-15364, (2012).

29 Theillet, F. X. et al. Structural disorder of monomeric alpha-synuclein persists in mammalian cells. Nature 530, 45-50, (2016).

30 Bertoncini, C. W. et al. Release of long-range tertiary interactions potentiates aggregation of natively unstructured alpha-synuclein. Proc Natl Acad Sci U S A 102, 1430-1435, (2005).

31 Allison, J. R., Varnai, P., Dobson, C. M. \& Vendruscolo, M. Determination of the free energy landscape of alpha-synuclein using spin label nuclear magnetic resonance measurements. J Am Chem Soc 131, 18314-18326, (2009).

32 Ulmer, T. S., Bax, A., Cole, N. B. \& Nussbaum, R. L. Structure and dynamics of micelle-bound human alpha-synuclein. J Biol Chem 280, 9595-9603, (2005).

33 Fusco, G. et al. Structural basis of synaptic vesicle assembly promoted by alpha-synuclein. Nat Commun 7, 12563, (2016).

34 Dobson, C. M. Protein folding and misfolding. Nature 426, 884-890, (2003).

35 Sunde, M. \& Blake, C. The structure of amyloid fibrils by electron microscopy and X-ray diffraction. Adv Protein Chem 50, 123-159, (1997).

36 Astbury, W. T., Dickinson, S. \& Bailey, K. The X-ray interpretation of denaturation and the structure of the seed globulins. Biochem $J$ 29, 2351-2360 2351, (1935). 
37 Bradbury, E. M. et al. The "Cross- $\beta$ " structure in polypeptides of low molecular weight. Journal of Molecular Biology 2, 276-IN271, (1960).

38 Hoyer, W. et al. Dependence of alpha-synuclein aggregate morphology on solution conditions. J Mol Biol 322, 383-393, (2002).

39 Uversky, V. N. A protein-chameleon: conformational plasticity of alphasynuclein, a disordered protein involved in neurodegenerative disorders. J Biomol Struct Dyn 21, 211-234, (2003).

40 Fernandez, C. O. et al. NMR of alpha-synuclein-polyamine complexes elucidates the mechanism and kinetics of induced aggregation. EMBO J 23, 2039-2046, (2004).

$41 \mathrm{Kim}, \mathrm{H}$. Y. et al. Structural properties of pore-forming oligomers of alphasynuclein. J Am Chem Soc 131, 17482-17489, (2009).

42 Henderson, M. X. et al. Unbiased Proteomics of Early Lewy Body Formation Model Implicates Active Microtubule Affinity-Regulating Kinases (MARKs) in Synucleinopathies. J Neurosci 37, 5870-5884, (2017).

43 Cookson, M. R. alpha-Synuclein and neuronal cell death. Mol Neurodegener 4, 9, (2009).

44 Bengoa-Vergniory, N., Roberts, R. F., Wade-Martins, R. \& AlegreAbarrategui, J. Alpha-synuclein oligomers: a new hope. Acta Neuropathol 134, 819-838, (2017).

45 Cox, D., Carver, J. A. \& Ecroyd, H. Preventing alpha-synuclein aggregation: the role of the small heat-shock molecular chaperone proteins. Biochim Biophys Acta 1842, 1830-1843, (2014).

46 Lee, S. J., Jeon, H. \& Kandror, K. V. Alpha-synuclein is localized in a subpopulation of rat brain synaptic vesicles. Acta Neurobiol Exp (Wars) 68, 509-515, (2008).

47 Vargas, K. J. et al. Synucleins regulate the kinetics of synaptic vesicle endocytosis. J Neurosci 34, 9364-9376, (2014).

48 George, J. M., Jin, H., Woods, W. S. \& Clayton, D. F. Characterization of a novel protein regulated during the critical period for song learning in the zebra finch. Neuron 15, 361-372, (1995).

49 Burre, J. et al. Alpha-synuclein promotes SNARE-complex assembly in vivo and in vitro. Science 329, 1663-1667, (2010).

50 Burre, J., Sharma, M. \& Sudhof, T. C. alpha-Synuclein assembles into higher-order multimers upon membrane binding to promote SNARE complex formation. Proc Natl Acad Sci U S A 111, E4274-4283, (2014).

51 Spillantini, M. G. et al. Alpha-synuclein in Lewy bodies. Nature 388, 839840, (1997).

52 Biere, A. L. et al. Parkinson's disease-associated alpha-synuclein is more fibrillogenic than beta- and gamma-synuclein and cannot crossseed its homologs. J Biol Chem 275, 34574-34579, (2000).

53 Nakazato, Y., Yamazaki, H., Hirato, J., Ishida, Y. \& Yamaguchi, H. Oligodendroglial microtubular tangles in olivopontocerebellar atrophy. $J$ Neuropathol Exp Neurol 49, 521-530, (1990).

54 Spillantini, M. G. et al. Filamentous alpha-synuclein inclusions link multiple system atrophy with Parkinson's disease and dementia with Lewy bodies. Neurosci Lett 251, 205-208, (1998).

55 Tretiakoff, C. Contribution a l'etude de l'Anatomie pathologique du Locus Niger de Soemmering avec quelques deduction relatives a la pathogenie 
des troubles du tonus musculaire et de la maladie de Parkinson. Theses de Paris, (1919).

56 Okazaki, H., Lipkin, L. E. \& Aronson, S. M. Diffuse intracytoplasmic ganglionic inclusions (Lewy type) associated with progressive dementia and quadriparesis in flexion. J Neuropathol Exp Neurol 20, 237-244, (1961).

57 Kalia, L. V. \& Lang, A. E. Parkinson disease in 2015: Evolving basic, pathological and clinical concepts in PD. Nat Rev Neurol 12, 65-66, (2016).

58 Alafuzoff, I. \& Hartikainen, P. Alpha-synucleinopathies. Handb Clin Neurol 145, 339-353, (2017).

59 McCann, H., Stevens, C. H., Cartwright, H. \& Halliday, G. M. alphaSynucleinopathy phenotypes. Parkinsonism Relat Disord 20 Suppl 1, S62-67, (2014).

60 Anderson, J. P. et al. Phosphorylation of Ser-129 is the dominant pathological modification of alpha-synuclein in familial and sporadic Lewy body disease. J Biol Chem 281, 29739-29752, (2006).

61 Hasegawa, M. et al. Phosphorylated alpha-synuclein is ubiquitinated in alpha-synucleinopathy lesions. J Biol Chem 277, 49071-49076, (2002).

62 Wakabayashi, K. et al. The Lewy body in Parkinson's disease and related neurodegenerative disorders. Mol Neurobiol 47, 495-508, (2013).

63 Xia, Q. et al. Proteomic identification of novel proteins associated with Lewy bodies. Front Biosci 13, 3850-3856, (2008).

64 Hirtz, D. et al. How common are the "common" neurologic disorders? Neurology 68, 326-337, (2007).

65 Wirdefeldt, K., Adami, H. O., Cole, P., Trichopoulos, D. \& Mandel, J. Epidemiology and etiology of Parkinson's disease: a review of the evidence. Eur J Epidemiol 26 Suppl 1, S1-58, (2011).

66 Dorsey, E. R. et al. Projected number of people with Parkinson disease in the most populous nations, 2005 through 2030. Neurology 68, 384386, (2007).

67 Gibb, W. R. \& Lees, A. J. The relevance of the Lewy body to the pathogenesis of idiopathic Parkinson's disease. J Neurol Neurosurg Psychiatry 51, 745-752, (1988).

68 Kruger, R. et al. Ala30Pro mutation in the gene encoding alpha-synuclein in Parkinson's disease. Nat Genet 18, 106-108, (1998).

69 Zarranz, J. J. et al. The new mutation, E46K, of alpha-synuclein causes Parkinson and Lewy body dementia. Ann Neurol 55, 164-173, (2004).

70 Appel-Cresswell, S. et al. Alpha-synuclein p.H50Q, a novel pathogenic mutation for Parkinson's disease. Mov Disord 28, 811-813, (2013).

71 Kiely, A. P. et al. alpha-Synucleinopathy associated with G51D SNCA mutation: a link between Parkinson's disease and multiple system atrophy? Acta Neuropathol 125, 753-769, (2013).

72 Polymeropoulos, M. H. et al. Mutation in the alpha-synuclein gene identified in families with Parkinson's disease. Science 276, 2045-2047, (1997).

73 Proukakis, C. et al. A novel alpha-synuclein missense mutation in Parkinson disease. Neurology 80, 1062-1064, (2013). 
74 Chartier-Harlin, M. C. et al. Alpha-synuclein locus duplication as a cause of familial Parkinson's disease. Lancet 364, 1167-1169, (2004).

75 Singleton, A. B. et al. alpha-Synuclein locus triplication causes Parkinson's disease. Science 302, 841, (2003).

76 Connolly, B. S. \& Lang, A. E. Pharmacological treatment of Parkinson disease: a review. JAMA 311, 1670-1683, (2014).

77 Williams, A. et al. Deep brain stimulation plus best medical therapy versus best medical therapy alone for advanced Parkinson's disease (PD SURG trial): a randomised, open-label trial. Lancet Neurol 9, 581591, (2010).

78 Heidebrink, J. L. Is dementia with Lewy bodies the second most common cause of dementia? J Geriatr Psychiatry Neurol 15, 182-187, (2002).

79 Gilman, S. et al. Second consensus statement on the diagnosis of multiple system atrophy. Neurology 71, 670-676, (2008).

80 Asi, Y. T. et al. Alpha-synuclein mRNA expression in oligodendrocytes in MSA. Glia 62, 964-970, (2014).

81 Peng, C., Gathagan, R. J. \& Lee, V. M. Distinct alpha-Synuclein strains and implications for heterogeneity among alpha-Synucleinopathies. Neurobiol Dis 109, 209-218, (2018).

82 Uchikado, H., Lin, W. L., DeLucia, M. W. \& Dickson, D. W. Alzheimer disease with amygdala Lewy bodies: a distinct form of alphasynucleinopathy. J Neuropathol Exp Neurol 65, 685-697, (2006).

83 Hamilton, R. L. Lewy bodies in Alzheimer's disease: a neuropathological review of 145 cases using alpha-synuclein immunohistochemistry. Brain Pathol 10, 378-384, (2000).

84 Liao, Y. C., Lebo, R. V., Clawson, G. A. \& Smuckler, E. A. Human prion protein cDNA: molecular cloning, chromosomal mapping, and biological implications. Science 233, 364-367, (1986).

85 Schmitt-Ulms, G., Ehsani, S., Watts, J. C., Westaway, D. \& Wille, H. Evolutionary descent of prion genes from the ZIP family of metal ion transporters. PLoS One 4, e7208, (2009).

86 Riek, R. et al. NMR structure of the mouse prion protein domain $\operatorname{PrP}(121-231)$. Nature 382, 180-182, (1996).

87 Haire, L. F. et al. The crystal structure of the globular domain of sheep prion protein. J Mol Biol 336, 1175-1183, (2004).

88 Smirnovas, V. et al. Structural organization of brain-derived mammalian prions examined by hydrogen-deuterium exchange. Nat Struct Mol Biol 18, 504-506, (2011).

89 Gambetti, P., Kong, Q., Zou, W., Parchi, P. \& Chen, S. G. Sporadic and familial CJD: classification and characterisation. Br Med Bull 66, 213239, (2003).

90 Goldfarb, L. G., Korczyn, A. D., Brown, P., Chapman, J. \& Gajdusek, D. C. Mutation in codon 200 of scrapie amyloid precursor gene linked to Creutzfeldt-Jakob disease in Sephardic Jews of Libyan and non-Libyan origin. Lancet 336, 637-638, (1990).

91 George, S. \& Brundin, P. Solving the conundrum of insoluble protein aggregates. Lancet Neurol 16, 258-259, (2017).

92 Powell-Jackson, J. et al. Creutzfeldt-Jakob disease after administration of human growth hormone. Lancet 2, 244-246, (1985). 
93 Hill, A. F. et al. The same prion strain causes vCJD and BSE. Nature 389, 448-450, 526, (1997).

94 Urwin, P. J., Mackenzie, J. M., Llewelyn, C. A., Will, R. G. \& Hewitt, P. E. Creutzfeldt-Jakob disease and blood transfusion: updated results of the UK Transfusion Medicine Epidemiology Review Study. Vox Sang 110, 310-316, (2016).

95 Safar, J. et al. Eight prion strains have $\operatorname{PrP}(\mathrm{Sc})$ molecules with different conformations. Nat Med 4, 1157-1165, (1998).

96 Tamguney, G. \& Korczyn, A. D. A critical review of the prion hypothesis of human synucleinopathies. Cell Tissue Res 373, 213-220, (2018).

97 Hashimoto, M. et al. Human recombinant NACP/alpha-synuclein is aggregated and fibrillated in vitro: relevance for Lewy body disease. Brain Res 799, 301-306, (1998).

98 Wood, S. J. et al. alpha-synuclein fibrillogenesis is nucleationdependent. Implications for the pathogenesis of Parkinson's disease. $J$ Biol Chem 274, 19509-19512, (1999).

99 Narkiewicz, J., Giachin, G. \& Legname, G. In vitro aggregation assays for the characterization of alpha-synuclein prion-like properties. Prion $\mathbf{8}$, 19-32, (2014).

100 Buell, A. K. et al. Solution conditions determine the relative importance of nucleation and growth processes in alpha-synuclein aggregation. Proc Natl Acad Sci U S A 111, 7671-7676, (2014).

101 Castilla, J., Saa, P., Hetz, C. \& Soto, C. In vitro generation of infectious scrapie prions. Cell 121, 195-206, (2005).

102 Atarashi, R., Sano, K., Satoh, K. \& Nishida, N. Real-time quakinginduced conversion: a highly sensitive assay for prion detection. Prion $\mathbf{5}$, 150-153, (2011).

103 Saborio, G. P., Permanne, B. \& Soto, C. Sensitive detection of pathological prion protein by cyclic amplification of protein misfolding. Nature 411, 810-813, (2001).

104 Fairfoul, G. et al. Alpha-synuclein RT-QuIC in the CSF of patients with alpha-synucleinopathies. Ann Clin Transl Neurol 3, 812-818, (2016).

105 Herva, M. E. et al. Anti-amyloid compounds inhibit alpha-synuclein aggregation induced by protein misfolding cyclic amplification (PMCA). J Biol Chem 289, 11897-11905, (2014).

106 Jung, B. C. et al. Amplification of distinct alpha-synuclein fibril conformers through protein misfolding cyclic amplification. Exp Mol Med 49, e314, (2017).

107 Braak, H. \& Braak, E. Neuropathological stageing of Alzheimer-related changes. Acta Neuropathol 82, 239-259, (1991).

108 Braak, H. et al. Staging of brain pathology related to sporadic Parkinson's disease. Neurobiol Aging 24, 197-211, (2003).

109 Braak, H. \& Del Tredici, K. Neuropathological Staging of Brain Pathology in Sporadic Parkinson's disease: Separating the Wheat from the Chaff. J Parkinsons Dis 7, S71-S85, (2017).

110 McKeith, I. G. et al. Consensus guidelines for the clinical and pathologic diagnosis of dementia with Lewy bodies (DLB): report of the consortium on DLB international workshop. Neurology 47, 1113-1124, (1996). 
111 Beach, T. G. et al. Unified staging system for Lewy body disorders: correlation with nigrostriatal degeneration, cognitive impairment and motor dysfunction. Acta Neuropathol 117, 613-634, (2009).

112 Goedert, M., Spillantini, M. G., Del Tredici, K. \& Braak, H. 100 years of Lewy pathology. Nat Rev Neurol 9, 13-24, (2013).

113 Braak, H., Rub, U., Gai, W. P. \& Del Tredici, K. Idiopathic Parkinson's disease: possible routes by which vulnerable neuronal types may be subject to neuroinvasion by an unknown pathogen. J Neural Transm (Vienna) 110, 517-536, (2003).

114 Kordower, J. H., Chu, Y., Hauser, R. A., Freeman, T. B. \& Olanow, C. W. Lewy body-like pathology in long-term embryonic nigral transplants in Parkinson's disease. Nat Med 14, 504-506, (2008).

$115 \mathrm{Li}, \mathrm{J}$. Y. et al. Lewy bodies in grafted neurons in subjects with Parkinson's disease suggest host-to-graft disease propagation. Nat Med 14, 501503, (2008).

116 Steiner, J. A., Quansah, E. \& Brundin, P. The concept of alpha-synuclein as a prion-like protein: ten years after. Cell Tissue Res 373, 161-173, (2018).

117 Peelaerts, W. et al. alpha-Synuclein strains cause distinct synucleinopathies after local and systemic administration. Nature 522, 340-344, (2015).

118 Abdelmotilib, H. et al. alpha-Synuclein fibril-induced inclusion spread in rats and mice correlates with dopaminergic Neurodegeneration. Neurobiol Dis 105, 84-98, (2017).

119 Rey, N. L. et al. Widespread transneuronal propagation of alphasynucleinopathy triggered in olfactory bulb mimics prodromal Parkinson's disease. J Exp Med 213, 1759-1778, (2016).

120 Stopschinski, B. E. \& Diamond, M. I. The prion model for progression and diversity of neurodegenerative diseases. Lancet Neurol 16, 323332, (2017).

121 Guo, J. L. et al. Distinct alpha-synuclein strains differentially promote tau inclusions in neurons. Cell 154, 103-117, (2013).

122 Bousset, L. et al. Structural and functional characterization of two alphasynuclein strains. Nat Commun 4, 2575, (2013).

123 Beekes, M., Thomzig, A., Schulz-Schaeffer, W. J. \& Burger, R. Is there a risk of prion-like disease transmission by Alzheimer- or Parkinsonassociated protein particles? Acta Neuropathol 128, 463-476, (2014).

124 Prusiner, S. B. et al. Evidence for alpha-synuclein prions causing multiple system atrophy in humans with parkinsonism. Proc Natl Acad Sci U S A 112, E5308-5317, (2015).

125 Bernis, M. E. et al. Prion-like propagation of human brain-derived alphasynuclein in transgenic mice expressing human wild-type alphasynuclein. Acta Neuropathol Commun 3, 75, (2015).

126 Vassar, P. S. \& Culling, C. F. Fluorescent stains, with special reference to amyloid and connective tissues. Arch Pathol 68, 487-498, (1959).

127 Puchtler, H., Sweat, F. \& Levine, M. On the binding of Congo red by amyloid. Journal of Histochemistry \& Cytochemistry 10, 355-364, (1962).

128 Higuchi, M. et al. 19F and 1H MRI detection of amyloid beta plaques in vivo. Nat Neurosci 8, 527-533, (2005). 
129 Velasco, A. et al. Detection of filamentous tau inclusions by the fluorescent Congo red derivative FSB [(trans,trans)-1-fluoro-2,5-bis(3hydroxycarbonyl-4-hydroxy)styrylbenzene]. FEBS Lett 582, 901-906, (2008).

130 Condello, C. et al. Structural heterogeneity and intersubject variability of Abeta in familial and sporadic Alzheimer's disease. Proc Natl Acad Sci U S A 115, E782-E791, (2018).

131 Sigurdson, C. J. et al. Prion strain discrimination using luminescent conjugated polymers. Nat Methods 4, 1023-1030, (2007).

132 Klingstedt, T. et al. The structural basis for optimal performance of oligothiophene-based fluorescent amyloid ligands: conformational flexibility is essential for spectral assignment of a diversity of protein aggregates. Chemistry 19, 10179-10192, (2013).

133 Tuttle, M. D. et al. Solid-state NMR structure of a pathogenic fibril of fulllength human alpha-synuclein. Nat Struct Mol Biol 23, 409-415, (2016).

134 Guerrero-Ferreira, R. et al. Cryo-EM structure of alpha-synuclein fibrils. Elife 7, (2018).

$135 \mathrm{Li}, \mathrm{Y}$. et al. Amyloid fibril structure of alpha-synuclein determined by cryoelectron microscopy. Cell Res 28, 897-903, (2018).

136 Delaglio, F. et al. NMRPipe: a multidimensional spectral processing system based on UNIX pipes. J Biomol NMR 6, 277-293, (1995).

137 Jeschke, G. et al. DeerAnalysis2006 - a comprehensive software package for analyzing pulsed ELDOR data. Appl Magn Reson 30, 473498, (2006).

138 Worswick, S. G., Spencer, J. A., Jeschke, G. \& Kuprov, I. Deep neural network processing of DEER data. Sci Adv 4, eaat5218, (2018).

139 Vranken, W. F. et al. The CCPN data model for NMR spectroscopy: development of a software pipeline. Proteins 59, 687-696, (2005).

140 Johnson, M., Coulton, A. T., Geeves, M. A. \& Mulvihill, D. P. Targeted amino-terminal acetylation of recombinant proteins in E. coli. PLoS One 5, e15801, (2010).

$141 \mathrm{Xu}, \mathrm{R}$. Light scattering: A review of particle characterization applications. Particuology 18, 11-21, (2015).

142 Minton, A. P. Recent applications of light scattering measurement in the biological and biopharmaceutical sciences. Anal Biochem 501, 4-22, (2016).

143 Burre, J. et al. Properties of native brain alpha-synuclein. Nature 498, E4-6; discussion E6-7, (2013).

144 Naiki, H., Higuchi, K., Hosokawa, M. \& Takeda, T. Fluorometric determination of amyloid fibrils in vitro using the fluorescent dye, thioflavin T1. Anal Biochem 177, 244-249, (1989).

145 Greenfield, N. J. Using circular dichroism spectra to estimate protein secondary structure. Nat Protoc 1, 2876-2890, (2006).

146 Farid, R. S. Circular Dichroism (CD) Spectroscopy, www.proteinchemist.com/cd/cdspec.html, (2003).

147 Klingstedt, T. et al. Distinct Spacing Between Anionic Groups: An Essential Chemical Determinant for Achieving Thiophene-Based Ligands to Distinguish beta-Amyloid or Tau Polymorphic Aggregates. Chemistry 21, 9072-9082, (2015). 
148 Bodenhausen, G. \& Ruben, D. J. Natural abundance nitrogen-15 NMR by enhanced heteronuclear spectroscopy. Chemical Physics Letters 69, 185-189, (1980).

149 Palmer, A. G., Cavanagh, J., Wright, P. E. \& Rance, M. Sensitivity improvement in proton-detected two-dimensional heteronuclear correlation NMR spectroscopy. Journal of Magnetic Resonance (1969) 93, 151-170, (1991).

150 Kay, L., Keifer, P. \& Saarinen, T. Pure absorption gradient enhanced heteronuclear single quantum correlation spectroscopy with improved sensitivity. Journal of the American Chemical Society 114, 10663-10665, (1992).

151 Schleucher, J. et al. A general enhancement scheme in heteronuclear multidimensional NMR employing pulsed field gradients. Journal of Biomolecular NMR 4, 301-306, (1994).

152 Grzesiek, S. \& Bax, A. Improved 3D triple-resonance NMR techniques applied to a $31 \mathrm{kDa}$ protein. Journal of Magnetic Resonance (1969) 96, 432-440, (1992).

153 Schleucher, J., Sattler, M. \& Griesinger, C. Coherence Selection by Gradients without Signal Attenuation: Application to the ThreeDimensional HNCO Experiment. Angewandte Chemie International Edition in English 32, 1489-1491, (1993).

154 Kay, L. E., Xu, G. Y. \& Yamazaki, T. Enhanced-Sensitivity TripleResonance Spectroscopy with Minimal H2O Saturation. Journal of Magnetic Resonance, Series A 109, 129-133, (1994).

155 Clubb, R. T., Thanabal, V. \& Wagner, G. A constant-time threedimensional triple-resonance pulse scheme to correlate intraresidue $1 \mathrm{HN}, 15 \mathrm{~N}$, and 13C' chemical shifts in 15N-13C-labelled proteins. Journal of Magnetic Resonance (1969) 97, 213-217, (1992).

156 Wittekind, M. \& Mueller, L. HNCACB, a High-Sensitivity 3D NMR Experiment to Correlate Amide-Proton and Nitrogen Resonances with the Alpha- and Beta-Carbon Resonances in Proteins. Journal of Magnetic Resonance, Series B 101, 201-205, (1993).

157 Muhandiram, D. R. \& Kay, L. E. Gradient-Enhanced Triple-Resonance Three-Dimensional NMR Experiments with Improved Sensitivity. Journal of Magnetic Resonance, Series B 103, 203-216, (1994).

158 Bax, A. Multidimensional Nuclear-Magnetic-Resonance Methods for Protein Studies. Curr Opin Struc Biol 4, 738-744, (1994).

159 Mobli, M. \& Hoch, J. C. Nonuniform sampling and non-Fourier signal processing methods in multidimensional NMR. Prog Nucl Magn Reson Spectrosc 83, 21-41, (2014).

160 Ying, J., Delaglio, F., Torchia, D. A. \& Bax, A. Sparse multidimensional iterative lineshape-enhanced (SMILE) reconstruction of both nonuniformly sampled and conventional NMR data. J Biomol NMR 68, 101118, (2017).

161 Schwarzinger, S. et al. Sequence-dependent correction of random coil NMR chemical shifts. J Am Chem Soc 123, 2970-2978, (2001).

162 Cho, M. K., Kim, H. Y., Fernandez, C. O., Becker, S. \& Zweckstetter, M. Conserved core of amyloid fibrils of wild type and A30P mutant alphasynuclein. Protein Sci 20, 387-395, (2011). 
163 Hoshino, M. et al. Mapping the core of the beta(2)-microglobulin amyloid fibril by H/D exchange. Nat Struct Biol 9, 332-336, (2002).

164 Vilar, M. et al. The fold of alpha-synuclein fibrils. Proc Natl Acad Sci U S A 105, 8637-8642, (2008).

165 Pornsuwan, S. et al. Long-range distances in amyloid fibrils of alphasynuclein from PELDOR spectroscopy. Angew Chem Int Ed Engl 52, 10290-10294, (2013).

166 Stoll, S. \& Schweiger, A. EasySpin, a comprehensive software package for spectral simulation and analysis in EPR. J Magn Reson 178, 42-55, (2006).

167 Jeschke, G. Conformational dynamics and distribution of nitroxide spin labels. Prog Nucl Magn Reson Spectrosc 72, 42-60, (2013).

168 Pannier, M., Veit, S., Godt, A., Jeschke, G. \& Spiess, H. W. Dead-time free measurement of dipole-dipole interactions between electron spins. Journal of Magnetic Resonance 142, 331-340, (2000).

169 Ree, R., Varland, S. \& Arnesen, T. Spotlight on protein N-terminal acetylation. Exp Mol Med 50, 90, (2018).

170 Bartels, T., Choi, J. G. \& Selkoe, D. J. alpha-Synuclein occurs physiologically as a helically folded tetramer that resists aggregation. Nature 477, 107-110, (2011).

171 Maltsev, A. S., Ying, J. \& Bax, A. Impact of N-terminal acetylation of alpha-synuclein on its random coil and lipid binding properties. Biochemistry 51, 5004-5013, (2012).

172 Miotto, M. C. et al. Copper binding to the N-terminally acetylated, naturally occurring form of alpha-synuclein induces local helical folding. J Am Chem Soc 137, 6444-6447, (2015).

$173 \mathrm{Li}, \mathrm{B}$. et al. Cryo-EM of full-length alpha-synuclein reveals fibril polymorphs with a common structural kernel. Nat Commun 9, 3609, (2018).

174 Krishna, M. M., Hoang, L., Lin, Y. \& Englander, S. W. Hydrogen exchange methods to study protein folding. Methods 34, 51-64, (2004).

175 Rodriguez, J. A. et al. Structure of the toxic core of alpha-synuclein from invisible crystals. Nature 525, 486-490, (2015).

176 Chen, M., Margittai, M., Chen, J. \& Langen, R. Investigation of alphasynuclein fibril structure by site-directed spin labeling. J Biol Chem 282, 24970-24979, (2007).

177 Toyama, B. H., Kelly, M. J., Gross, J. D. \& Weissman, J. S. The structural basis of yeast prion strain variants. Nature 449, 233-237, (2007).

178 Fitzpatrick, A. W. et al. Atomic structure and hierarchical assembly of a cross-beta amyloid fibril. Proc Natl Acad Sci U S A 110, 5468-5473, (2013).

179 Miake, H., Mizusawa, H., Iwatsubo, T. \& Hasegawa, M. Biochemical characterization of the core structure of alpha-synuclein filaments. $\mathrm{J} \mathrm{Biol}$ Chem 277, 19213-19219, (2002).

180 Crowther, R. A. Straight and paired helical filaments in Alzheimer disease have a common structural unit. Proc Natl Acad Sci U S A 88, 2288-2292, (1991).

181 Fitzpatrick, A. W. P. et al. Cryo-EM structures of tau filaments from Alzheimer's disease. Nature 547, 185-190, (2017). 
182 Jucker, M. \& Walker, L. C. Propagation and spread of pathogenic protein assemblies in neurodegenerative diseases. Nat Neurosci 21, 13411349, (2018).

183 Spillantini, M. G. \& Goedert, M. Tau pathology and neurodegeneration. Lancet Neurol 12, 609-622, (2013).

184 Falcon, B. et al. Structures of filaments from Pick's disease reveal a novel tau protein fold. Nature 561, 137-140, (2018).

185 Govaerts, C., Wille, H., Prusiner, S. B. \& Cohen, F. E. Evidence for assembly of prions with left-handed beta-helices into trimers. Proc Natl Acad Sci U S A 101, 8342-8347, (2004).

186 Peng, C. et al. Cellular milieu imparts distinct pathological alphasynuclein strains in alpha-synucleinopathies. Nature 557, 558-563, (2018).

187 Wang, J., Gu, B. J., Masters, C. L. \& Wang, Y. J. A systemic view of Alzheimer disease - insights from amyloid-beta metabolism beyond the brain. Nat Rev Neurol 13, 703, (2017).

188 Murphy, M. P. \& LeVine, H., 3rd. Alzheimer's disease and the amyloidbeta peptide. J Alzheimers Dis 19, 311-323, (2010).

189 Petkova, A. T. et al. Self-propagating, molecular-level polymorphism in Alzheimer's beta-amyloid fibrils. Science 307, 262-265, (2005).

190 Petkova, A. T., Yau, W. M. \& Tycko, R. Experimental constraints on quaternary structure in Alzheimer's beta-amyloid fibrils. Biochemistry 45, 498-512, (2006).

191 Paravastu, A. K., Leapman, R. D., Yau, W. M. \& Tycko, R. Molecular structural basis for polymorphism in Alzheimer's beta-amyloid fibrils. Proc Natl Acad Sci U S A 105, 18349-18354, (2008).

192 Sachse, C., Fandrich, M. \& Grigorieff, N. Paired beta-sheet structure of an Abeta(1-40) amyloid fibril revealed by electron microscopy. Proc Natl Acad Sci U S A 105, 7462-7466, (2008).

193 Lu, J. X. et al. Molecular structure of beta-amyloid fibrils in Alzheimer's disease brain tissue. Cell 154, 1257-1268, (2013).

194 Schmidt, M. et al. Peptide dimer structure in an Abeta(1-42) fibril visualized with cryo-EM. Proc Natl Acad Sci U S A 112, 11858-11863, (2015).

195 Xiao, Y. et al. Abeta(1-42) fibril structure illuminates self-recognition and replication of amyloid in Alzheimer's disease. Nat Struct Mol Biol 22, 499505, (2015).

196 Walti, M. A. et al. Atomic-resolution structure of a disease-relevant Abeta(1-42) amyloid fibril. Proc Natl Acad Sci U S A 113, E4976-4984, (2016).

197 Colvin, M. T. et al. Atomic Resolution Structure of Monomorphic Abeta42 Amyloid Fibrils. J Am Chem Soc 138, 9663-9674, (2016).

198 Gremer, L. et al. Fibril structure of amyloid-beta(1-42) by cryo-electron microscopy. Science 358, 116-119, (2017).

199 Qiang, W., Yau, W. M., Lu, J. X., Collinge, J. \& Tycko, R. Structural variation in amyloid-beta fibrils from Alzheimer's disease clinical subtypes. Nature 541, 217-221, (2017).

200 Wilkins, M. R. et al. Protein identification and analysis tools in the ExPASy server. Methods Mol Biol 112, 531-552, (1999). 
201 Spillantini, M. G., Divane, A. \& Goedert, M. Assignment of human alphasynuclein (SNCA) and beta-synuclein (SNCB) genes to chromosomes 4q21 and 5q35. Genomics 27, 379-381, (1995).

202 Masaracchia, C. et al. Membrane binding, internalization, and sorting of alpha-synuclein in the cell. Acta Neuropathol Commun 6, 79, (2018).

203 Miotto, M. C. et al. Bioinorganic Chemistry of Parkinson's Disease: Affinity and Structural Features of $\mathrm{Cu}(\mathrm{I})$ Binding to the Full-Length betaSynuclein Protein. Inorg Chem 56, 10387-10395, (2017).

204 Pfefferkorn, C. M. \& Lee, J. C. Tryptophan probes at the alpha-synuclein and membrane interface. J Phys Chem B 114, 4615-4622, (2010). 


\section{$7 \quad$ Appendix}

\subsection{Human wt $\alpha$-synuclein sequence}

\subsubsection{Fasta sequence from Uniprot}

>sp|P37840|SYUA_HUMAN Alpha-synuclein OS=Homo sapiens OX=9606 GN=SNCA $\mathrm{PE}=1 \quad \mathrm{SV}=1$

MDVFMKGLSKAKEGVVAAAEKTKQGVAEAAGKTKEGVLYVGSKTKEGVVHGVATVAEKTK

EQVTNVGGAVVTGVTAVAQKTVEGAGS IAAATGFVKKDQLGKNEEGAPQEGILEDMPVDP

DNEAYEMPSEEGYQDYEPEA

\subsubsection{Physical and chemical parameters of $\alpha$-synuclein}

Number of amino acids: $\quad 140$

Molecular weight:

$14,460 \mathrm{Da}$

Theoretical pl:

4.67

Additional weight due to $\mathrm{N}$-terminal acetylation: $42 \mathrm{Da}$

$\mathrm{H}_{3} \mathrm{C}-\mathrm{C}=\mathrm{O}: 43 \mathrm{Da}$

Molecular weight of aSyn: $\quad 14,502 \mathrm{Da}$

Data obtained using FASTA sequences and ProtParam of SIB ExPASy server ${ }^{200}$.

\subsection{Resonance assignment of aSyn in dissociation buffer}

$\begin{array}{lllllll}\# & \text { Residue } & \mathrm{H} & \mathrm{N} & \mathrm{C}^{\prime} & \mathrm{CA} & \mathrm{CB} \\ 1 & \text { Met } & 8.18 & 126.14 & 176.51 & 56.34 & 33.22 \\ 2 & \text { Asp } & 8.39 & 119.59 & 174.87 & 53 & 38.03 \\ 3 & \text { Val } & 7.74 & 119.46 & 175.78 & 62.87 & 32.77 \\ 4 & \text { Phe } & 8.07 & 123.09 & - & 58.17 & 39.6 \\ 5 & \text { Met } & 8.03 & 121.74 & 175.92 & 55.73 & 33.12 \\ 6 & \text { Lys } & 8.06 & 122.27 & 177.06 & 57.28 & 33.25 \\ 7 & \text { Gly } & 8.22 & 110.02 & 174.06 & 45.67 & - \\ 8 & \text { Leu } & 7.95 & 121.68 & 177.5 & 55.32 & 42.62 \\ 9 & \text { Ser } & 8.18 & 116.72 & 174.64 & 58.57 & 64.14 \\ 10 & \text { Lys } & 8.21 & 123.45 & 176.52 & 56.93 & 33.16 \\ 11 & \text { Ala } & 8.13 & 124.64 & - & 53.06 & 19.46 \\ 12 & \text { Lys } & 8.1 & 120.53 & 176.68 & 56.84 & 33.19 \\ 13 & \text { Glu } & 8.19 & 120.96 & 175.98 & 56.27 & -\end{array}$


7.86

119.61

176.41

62.7

33.08

17

18

19

20

21

22

23

26

27

28

29

30

31

32

33

34

35

37

38

39

40

41

42

43

44

45

47

48

49

50

51

52

53

54

Val

8.02

123.75

176.04

62.5

33.12

8.21

127.63

177.7

53.01

19.39

8.11

123.16

177.76

53.1

19.38

8.1

122.67

177.84

52.96

19.47

8.08

119.02

176.15

56.12

28.92

8.15

122.33

176.76

57

33.31

8.03

114.56

174.63

61.88

70.48

8.19

123.62

176.58

57.05

33.21

7.89

119.28

176.17

62.51

33.03

8.24

127.18

177.84

53.07

19.37

8.15

119.72

175.93

56.16

28.87

8.16

124.93

177.45

52.85

19.47

8.14

123.27

178.22

53.06

19.5

8.18

108.21

174.04

45.55

8.07

121.19

176.84

56.73

33.51

8.08

114.91

174.6

61.86

70.52

8.22

123.66

176.53

56.98

33.22

8.24

120.99

176.48

56.23

29.02

7.86

119.09

176.02

62.5

32.98

8.05

125.06

176.69

55.32

42.51

8

121.68

175.71

58.05

39

7.91

122.27

176.21

62.5

32.98

7.87

112.04

173.94

45.49

8.14

115.77

174.75

58.57

64.37

8.32

123.53

176.76

57.06

33.22

8.03

114.48

174.62

61.88

123.53

176.68

57.01

33.23

8.25

110.37

173.75

45.56

119.45

176.14

62.3

33.16

8.05

123.92

175.82

62.61

32.99

8.4

122.13

174.56

55.3

29.53

8.32

110.75

45.56

7.99

119.21

175.91

62.21

33.23

8.28

127.71

177.76

52.81

19.63

8

113.49

174.66

61.71

70.48 


\begin{tabular}{|c|c|c|c|c|c|c|}
\hline 55 & Val & 8.01 & 121.58 & 176.07 & 62.72 & 32.98 \\
\hline 56 & Ala & 8.21 & 127.09 & 177.76 & 53.06 & 19.46 \\
\hline 57 & Glu & 8.1 & 119.77 & 176.26 & 56.27 & 28.95 \\
\hline 58 & Lys & 8.22 & 122.31 & 176.89 & 57.03 & 33.36 \\
\hline 59 & Thr & 8.07 & 114.7 & 174.68 & 62.12 & 70.37 \\
\hline 60 & Lys & 8.16 & 123.32 & 176.7 & 57.16 & 33.2 \\
\hline 62 & Gln & 8.24 & 121.54 & 175.99 & 56.27 & 29.68 \\
\hline 63 & Val & 8.1 & 121.04 & 176.27 & 62.65 & 33.2 \\
\hline 64 & Thr & 8.09 & 116.79 & 174.1 & 61.75 & 70.33 \\
\hline 65 & Asn & 8.34 & 121.61 & 175.18 & 53.24 & 39.1 \\
\hline 66 & Val & 8.07 & 120.01 & 176.68 & 62.78 & 32.8 \\
\hline 67 & Gly & 8.34 & 112.33 & 174.58 & 45.6 & - \\
\hline 68 & Gly & 8.15 & 109.18 & 173.75 & 45.35 & - \\
\hline 69 & Ala & 8.12 & 124.16 & 177.64 & 52.72 & 19.66 \\
\hline 70 & Val & 8.02 & 119.87 & 176.2 & 62.59 & 32.96 \\
\hline 71 & Val & 8.11 & 124.3 & 176.17 & 62.35 & 32.97 \\
\hline 72 & Thr & 8.12 & 117.35 & 174.96 & 61.76 & 70.41 \\
\hline 73 & Gly & 8.24 & 111.29 & 173.94 & 45.55 & - \\
\hline 74 & Val & 7.96 & 119.19 & 176.43 & 62.53 & 33.09 \\
\hline 75 & Thr & 8.09 & 117.33 & 174.19 & 61.75 & 70.3 \\
\hline 76 & Ala & 8.17 & 126.68 & 177.57 & 52.88 & 19.61 \\
\hline 77 & Val & 7.93 & 118.99 & 175.98 & 62.42 & 33.07 \\
\hline 78 & Ala & 8.19 & 127.51 & 177.58 & 52.88 & 19.44 \\
\hline 79 & Gln & 8.21 & 120.04 & 175.95 & 56.16 & 29.78 \\
\hline 80 & Lys & 8.23 & 122.67 & 176.65 & 56.96 & 33.44 \\
\hline 81 & Thr & 8.09 & 115.42 & 174.5 & 61.86 & 70.45 \\
\hline 82 & Val & 8.05 & 122.05 & 176.15 & 62.52 & 33.02 \\
\hline 83 & Glu & 8.29 & 123.74 & 176.48 & 56.2 & 28.93 \\
\hline 84 & Gly & 8.28 & 110.62 & 173.89 & 45.54 & - \\
\hline 85 & Ala & 8.20 & 124.15 & 178.28 & 53.17 & 19.59 \\
\hline 86 & Gly & 8.34 & 108.53 & 174.13 & 45.49 & - \\
\hline 87 & Ser & 8.09 & 115.97 & 174.73 & 58.41 & 64.37 \\
\hline 88 & lle & 8.09 & 122.57 & 176.27 & 61.84 & 38.91 \\
\hline 89 & Ala & 8.18 & 127.51 & 177.64 & 52.97 & 19.44 \\
\hline 90 & Ala & 8.05 & 123.02 & 177.67 & 52.92 & 19.46 \\
\hline 91 & Ala & 8.13 & 123.26 & 177.91 & - & - \\
\hline
\end{tabular}




\begin{tabular}{|c|c|c|c|c|c|c|}
\hline 92 & Thr & 7.97 & 111.85 & 175.1 & 61.92 & 70.34 \\
\hline 93 & Gly & 8.16 & 110.91 & 173.5 & 45.44 & - \\
\hline 94 & Phe & 8.02 & 120.78 & 175.44 & 58.1 & 40.03 \\
\hline 95 & Val & 7.87 & 122.89 & 175.57 & 62.14 & 33.27 \\
\hline 96 & Lys & 8.15 & 125.86 & 176.66 & 56.96 & - \\
\hline 97 & Lys & 8.18 & 122.6 & 176.43 & 57.22 & 33.24 \\
\hline 98 & Asp & 8.29 & 119.29 & 175.03 & 53.2 & 38.13 \\
\hline 99 & Gln & 8.22 & 120.74 & 175.94 & 56.23 & 29.58 \\
\hline 100 & Leu & 8.05 & 122.63 & 177.84 & 55.59 & 42.41 \\
\hline 101 & Gly & 8.21 & 109.73 & 174.22 & 45.59 & - \\
\hline 102 & Lys & 8.09 & 121.06 & 176.52 & 57 & 33.45 \\
\hline 103 & Asn & 8.39 & 119.52 & 175.21 & 53.49 & 38.9 \\
\hline 107 & Ala & 8.07 & 124.88 & 175.62 & 50.91 & 18.44 \\
\hline 108 & Pro & - & - & 176.92 & 63.59 & 32.27 \\
\hline 109 & Gln & 8.37 & 120.53 & 175.88 & 56.16 & 29.56 \\
\hline 110 & Glu & 8.25 & 121.15 & 176.33 & 56.15 & 29.12 \\
\hline 111 & Gly & 8.29 & 110.43 & 173.96 & 45.61 & - \\
\hline 112 & Ile & 7.87 & 119.93 & 176.42 & 61.63 & 39 \\
\hline 113 & Leu & 8.09 & 125.34 & 177.36 & 55.52 & 42.03 \\
\hline 114 & Glu & 8.11 & 120.36 & 175.62 & 56.12 & 28.74 \\
\hline 115 & Asp & 8.25 & 119.48 & 174.45 & 52.83 & 38.33 \\
\hline 116 & Met & 8.05 & 121.37 & 174.09 & 53.7 & 32.68 \\
\hline 117 & Pro & - & - & 176.51 & 63.28 & 32.45 \\
\hline 118 & Val & 8.07 & 120.27 & 175.45 & 62.66 & 33 \\
\hline 119 & Asp & 8.38 & 122.39 & 173.15 & 51.05 & 38.34 \\
\hline 120 & Pro & - & - & 176.69 & 64.03 & 32.32 \\
\hline 121 & Asp & 8.23 & 117.78 & 174.89 & 53.15 & 37.96 \\
\hline 122 & Asn & 8.15 & 119.17 & 175.25 & 53.45 & 38.96 \\
\hline 123 & Glu & 8.1 & 120.3 & 175.79 & 56.87 & 28.68 \\
\hline 124 & Ala & 8.13 & 124.45 & 177.34 & 53.03 & 19.38 \\
\hline 125 & Tyr & 7.89 & 119.09 & 175.55 & 58.18 & 38.97 \\
\hline 126 & Glu & 7.96 & 121.61 & 175.16 & 55.45 & 29.3 \\
\hline 127 & Met & 8.14 & 122.55 & 174.36 & 53.74 & 32.71 \\
\hline 128 & Pro & - & - & 176.88 & 63.77 & 32.32 \\
\hline 129 & Ser & 8.27 & 115.92 & 174.74 & 58.67 & 64.06 \\
\hline 130 & Glu & 8.29 & 122.09 & 175.97 & 56.09 & 28.81 \\
\hline
\end{tabular}


Appendix

$\begin{array}{lllllll}131 & \text { Glu } & 8.27 & 120.85 & 176.42 & 56.3 & 28.89 \\ 132 & \text { Gly } & 8.21 & 110.11 & 173.85 & 45.48 & - \\ 133 & \text { Tyr } & 7.99 & 120.49 & 175.97 & 58.49 & 38.99 \\ 134 & \text { Gln } & 8.21 & 121.47 & 175.31 & 56.13 & 29.41 \\ 135 & \text { Asp } & 8.15 & 119.76 & 174.37 & 52.93 & 38.23 \\ 136 & \text { Tyr } & 7.98 & 121.3 & 175.11 & 58.18 & 39.07 \\ 137 & \text { Glu } & 8.00 & 123.23 & 173.53 & 53.3 & 28.75 \\ 138 & \text { Pro } & - & - & 176.7 & 63.45 & 32.35 \\ 139 & \text { Glu } & 8.26 & 120.15 & 175.57 & 55.88 & 28.94 \\ 140 & \text { Ala } & 8.19 & 126.58 & 179.39 & 52.04 & 19.22\end{array}$

Table 5. Resonance assignment of aSyn in dissociation buffer. 


\subsection{HS-68 synthesis report}

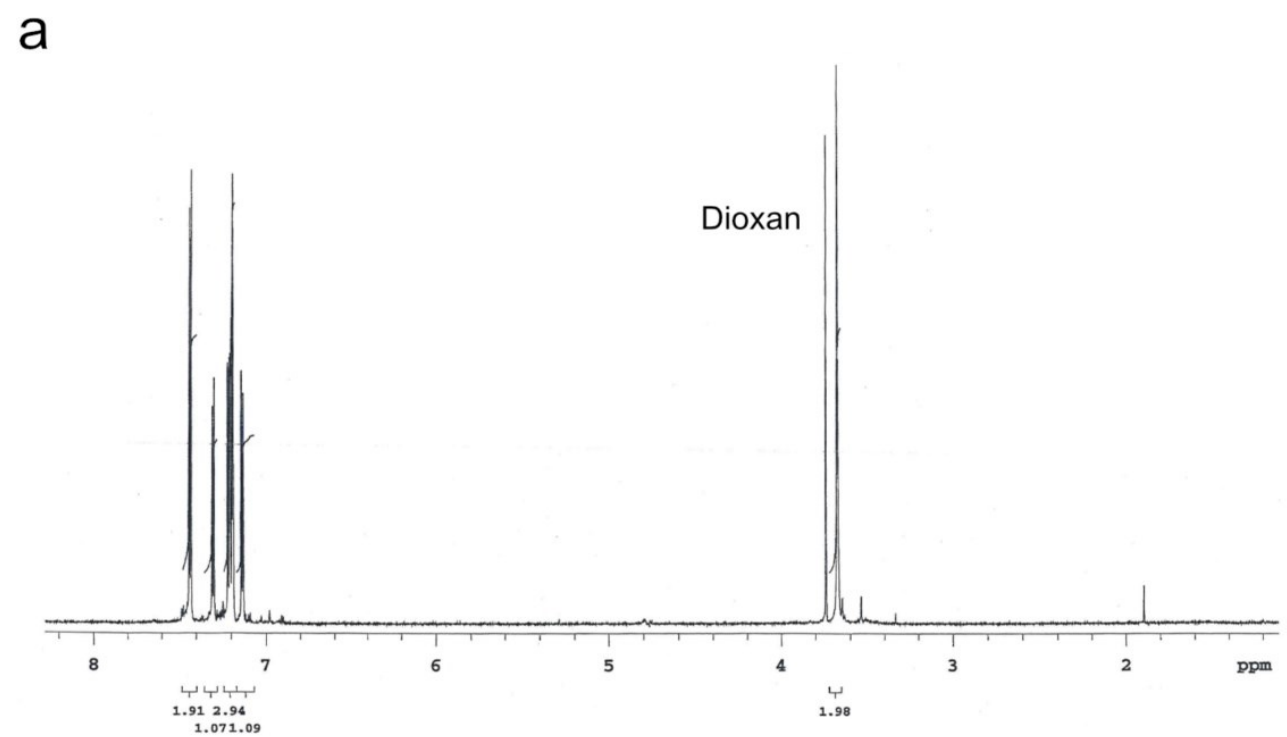

b

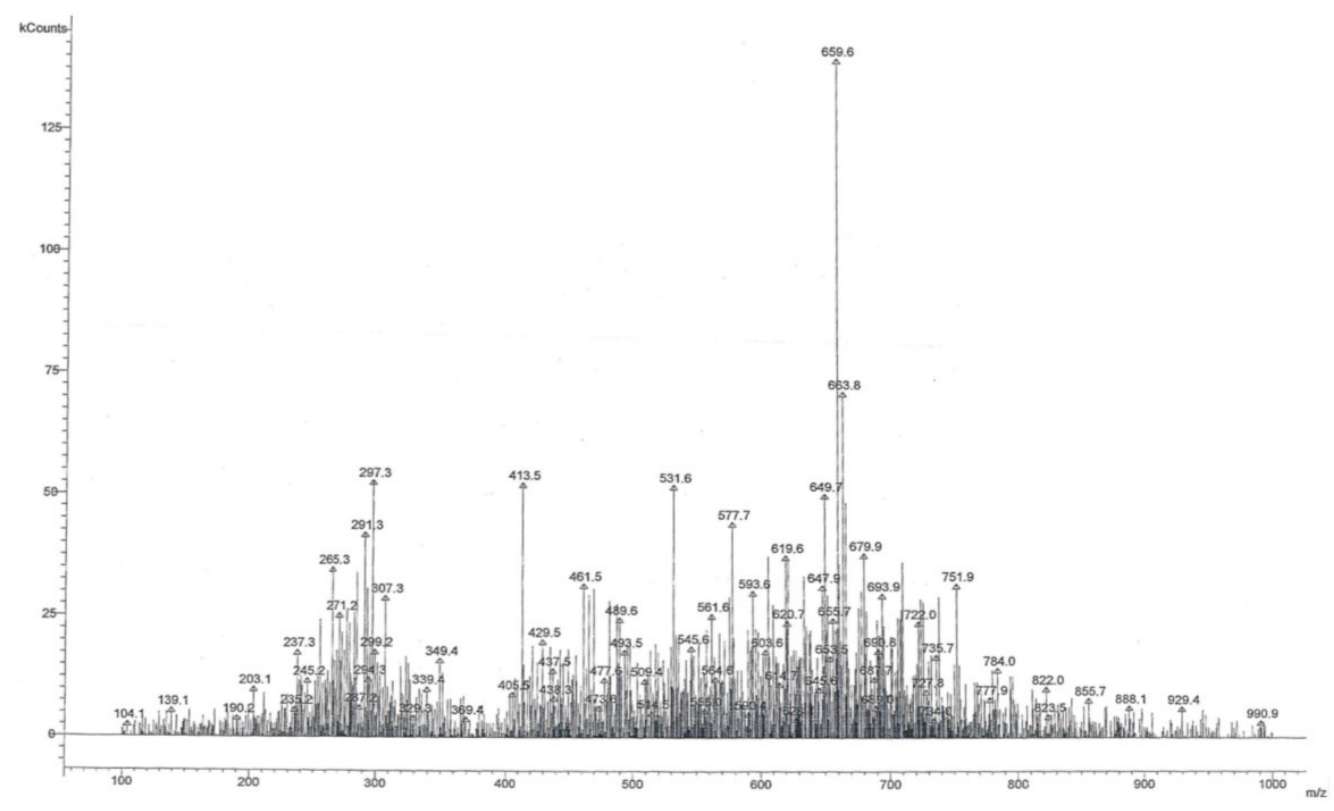

Figure 7-1. Synthesis report of HS-68.

(a) ${ }^{1} \mathrm{H}$ NMR spectrum and (b) mass spectrum of HS-68. 


\subsection{Mass spectrometry of non-acetylated $\alpha$-synuclein}

a
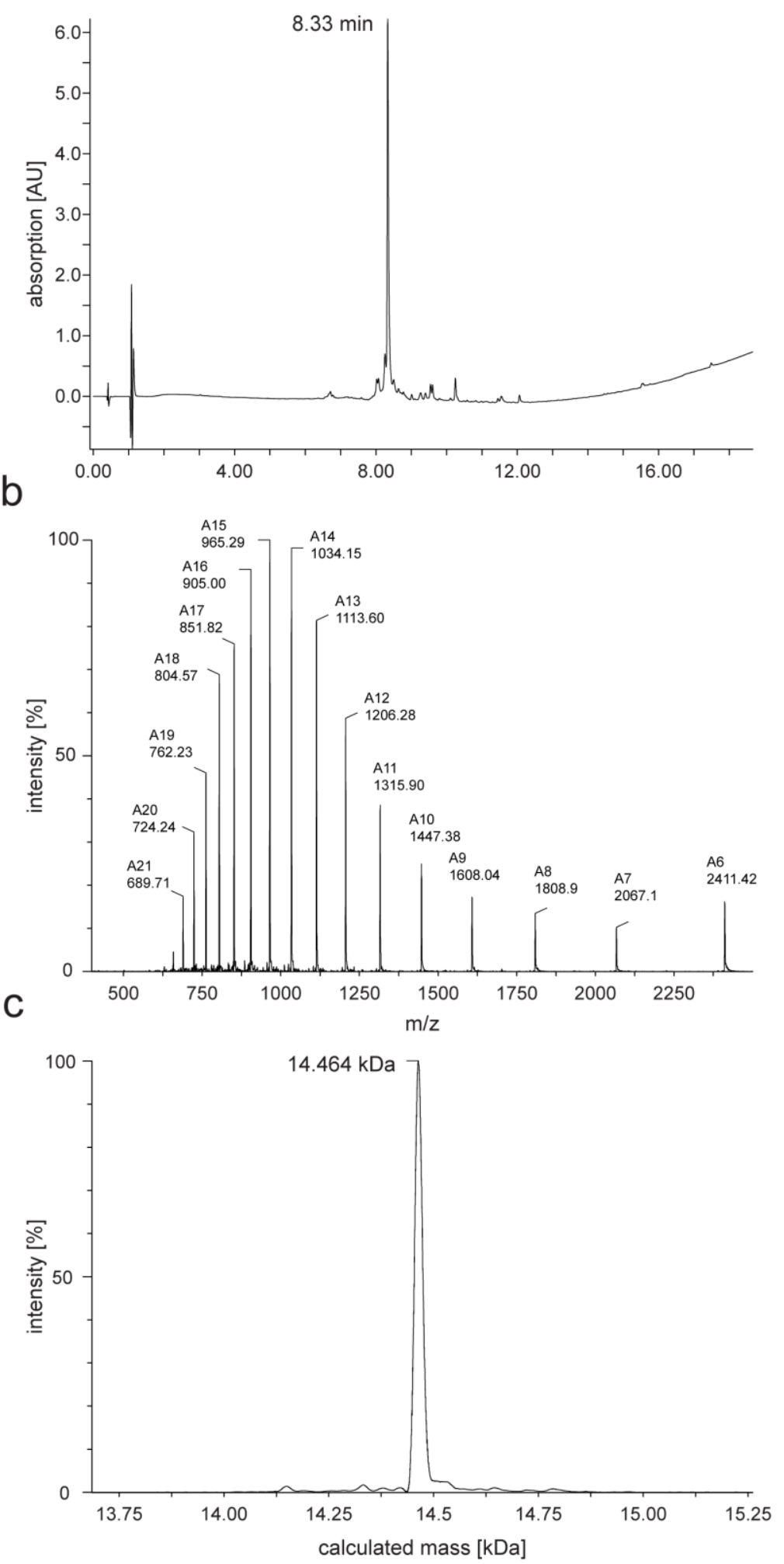

Figure 7-2. Mass spectrometry of non-acetylated $\alpha$-synuclein

(a) HPLC run of non-acetylated $\alpha$-synuclein. (b) M/z spectrum of $\alpha$-synuclein. (c) Calculated average mass for HPLC elution. 


\subsection{Pulse sequence used for real-time HD back-exchange}

\subsubsection{HSQC pulse program}

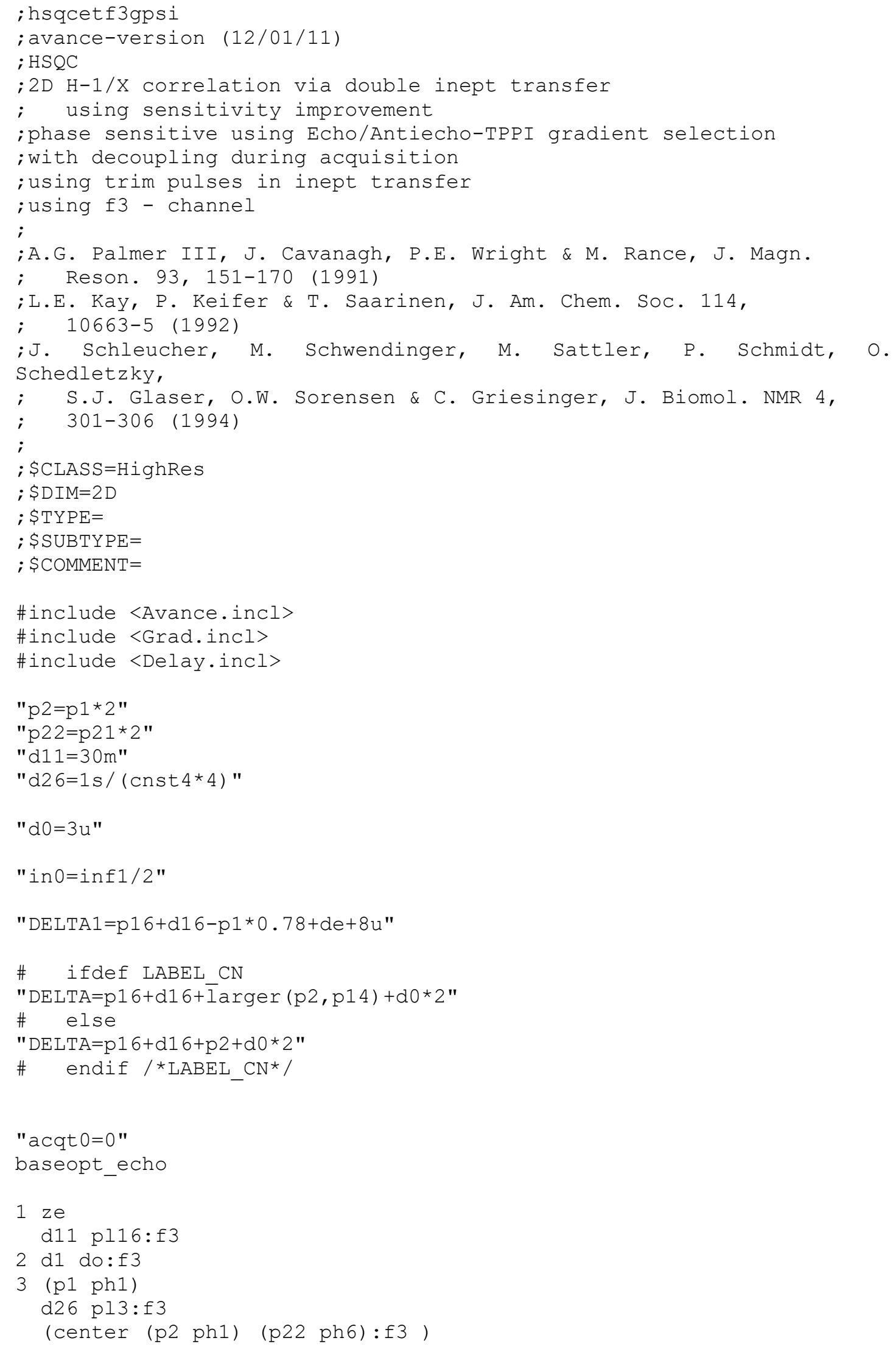




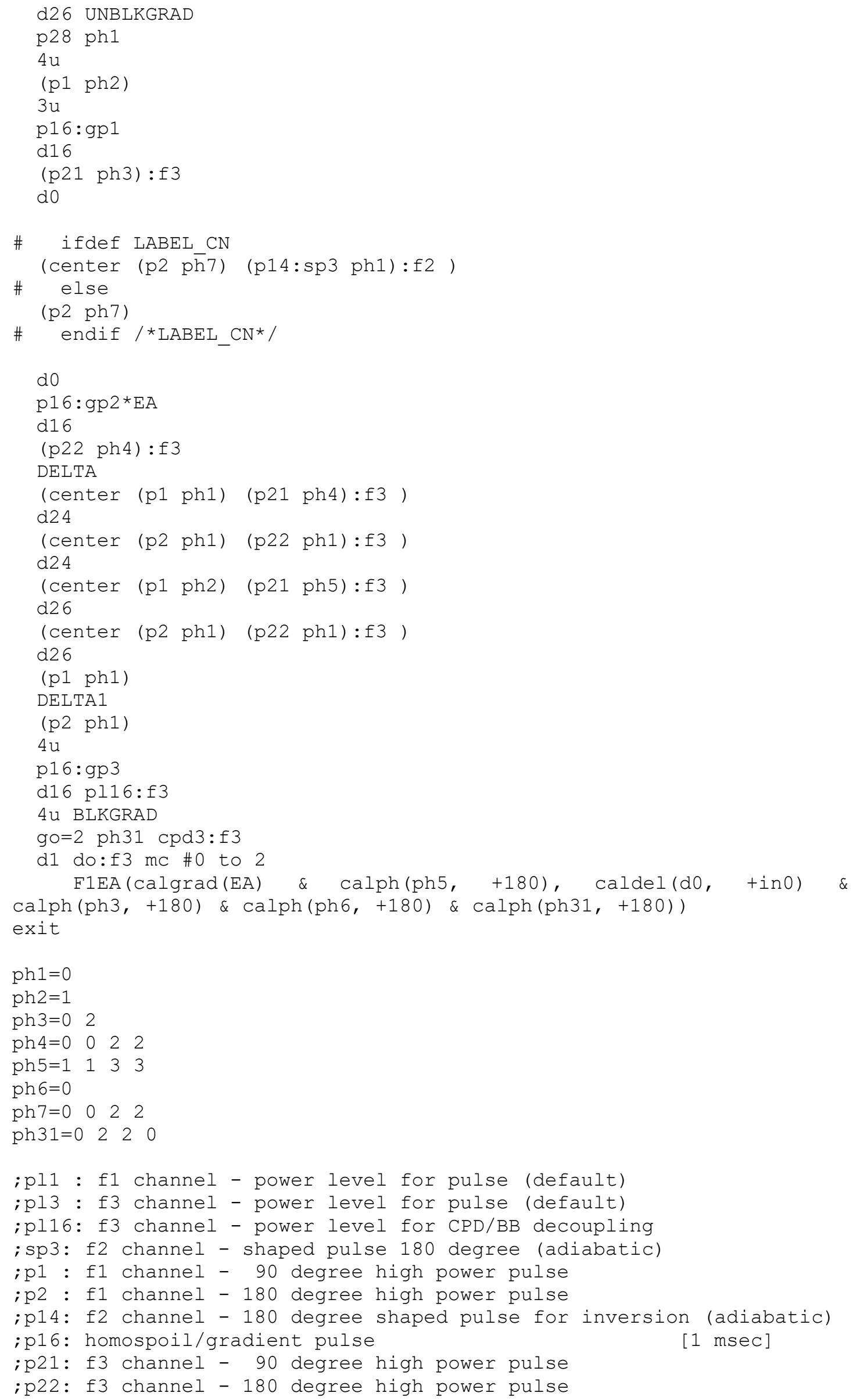




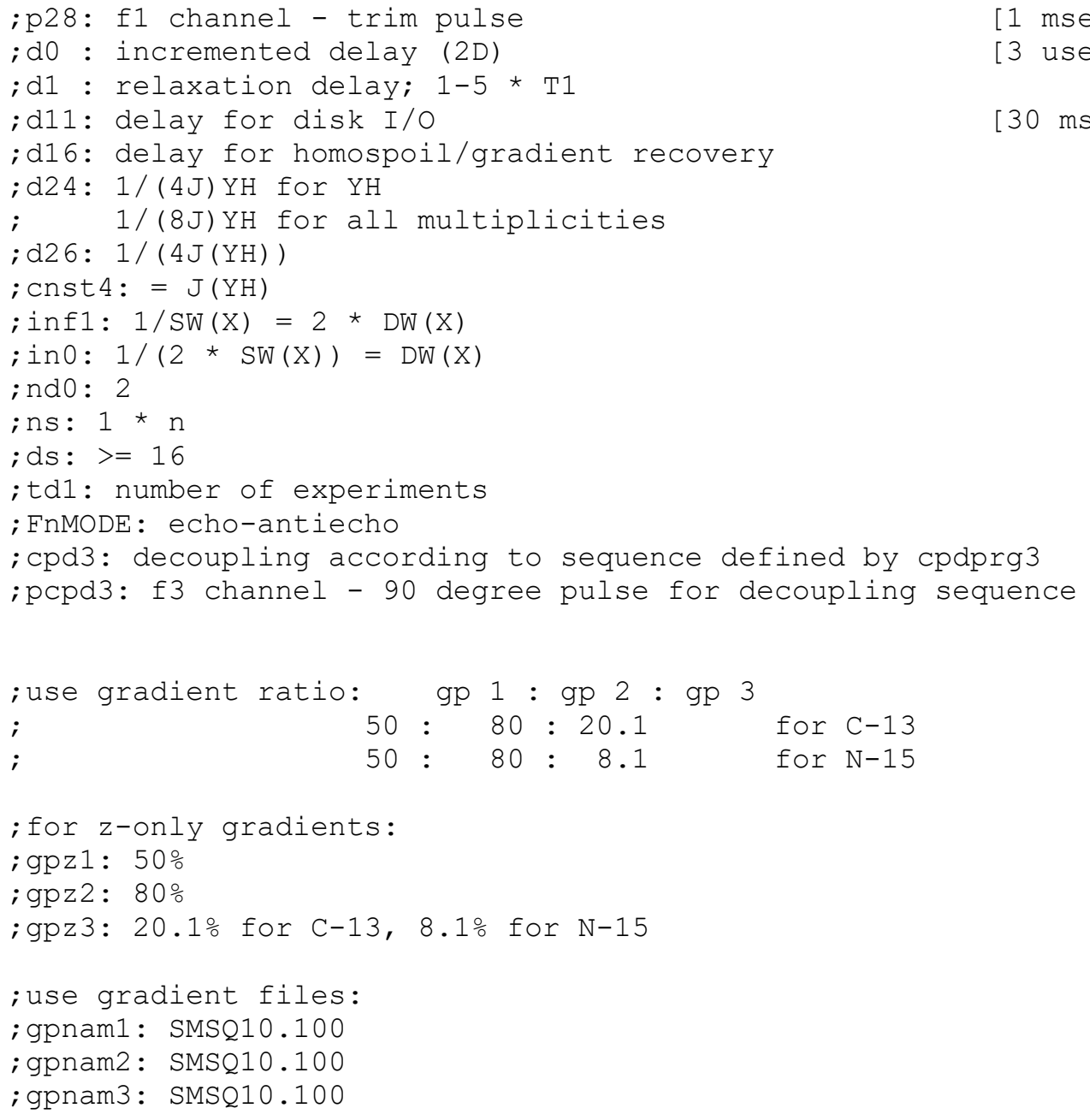

\subsubsection{HSQC acquisition parameters}

$\underline{A Q \bmod } \quad D Q D$

FnTYPE traditional(planes)

FnMODE Echo-Antiecho

TD $\quad F 2: 2048 \quad$ F1:128

DS $\quad 4$

NS $\quad 4$

SW [ppm] F2: $10.78 \quad F 1: 22.04$

O1P [ppm] F2: $4.69 \quad F 1: 118.00$

Table 6. HSQC acquisition parameters. 
7.6 Residue-specific protonation levels of aSyn fibrils

7.6.1 aSyn fibrils seeded by brain extract of patient brain

\begin{tabular}{|c|c|c|c|c|c|c|c|c|c|}
\hline ID & $\mathbf{A A}$ & PD1 & PD2 & PD3 & DLB1 & DLB2 & DLB1_rep & MSA1 & MSA2 \\
\hline 1 & $M$ & 30.1 & 24.3 & 36.6 & 30.1 & 34.0 & 35.8 & 18.4 & 33.3 \\
\hline 3 & V & 21.1 & 16.3 & 17.4 & 17.5 & 13.2 & 23.1 & 7.6 & 14.0 \\
\hline 4 & $\mathrm{~F}$ & 17.7 & 14.7 & 19.2 & 19.1 & 13.4 & 13.6 & 8.9 & 13.2 \\
\hline 5 & $M$ & 20.4 & 15.9 & 15.4 & 18.8 & 11.3 & 20.3 & 11.1 & 10.6 \\
\hline 6 & K & 28.5 & 22.0 & 25.2 & 19.1 & 19.1 & 24.9 & 16.8 & 19.9 \\
\hline 7 & G & 11.3 & 16.5 & 14.5 & 14.7 & 7.1 & 17.8 & 5.5 & 9.0 \\
\hline 9 & $S$ & 20.4 & 15.5 & 16.9 & 17.6 & 12.5 & 22.8 & 9.5 & 15.3 \\
\hline 11 & A & 9.9 & 8.6 & 12.9 & 8.3 & 5.7 & 8.7 & -0.3 & 11.8 \\
\hline 12 & K & 6.8 & 10.3 & 12.1 & 7.9 & 7.1 & 13.3 & 1.4 & 7.7 \\
\hline 15 & V & 27.2 & 26.5 & 38.0 & 38.9 & 34.7 & 38.2 & 47.7 & 46.1 \\
\hline 16 & v & 32.3 & 36.7 & 45.3 & 49.2 & 55.5 & 50.2 & 65.6 & 63.3 \\
\hline 17 & A & 36.2 & 38.2 & 49.2 & 53.1 & 56.1 & 55.9 & 68.0 & 66.2 \\
\hline 18 & A & 37.4 & 38.9 & 43.8 & 50.0 & 52.6 & 52.3 & 63.5 & 62.4 \\
\hline 19 & A & 32.0 & 42.6 & 38.5 & 46.4 & 46.4 & 45.6 & 59.1 & 52.4 \\
\hline 20 & $\mathrm{E}$ & 30.7 & 30.7 & 40.2 & 49.4 & 51.5 & 42.6 & 62.1 & 59.7 \\
\hline 21 & K & 30.3 & 28.0 & 39.0 & 48.0 & 45.9 & 41.4 & 60.5 & 58.8 \\
\hline 22 & $\mathrm{~T}$ & 29.4 & 32.9 & 39.5 & 43.8 & 44.1 & 43.8 & 66.4 & 54.9 \\
\hline 26 & V & 24.1 & 27.9 & 31.5 & 35.8 & 37.5 & 26.1 & 52.0 & 53.9 \\
\hline 27 & $A$ & 32.1 & 32.5 & 39.4 & 53.5 & 45.9 & 43.2 & 60.0 & 63.3 \\
\hline 29 & A & 29.9 & 33.4 & 37.2 & 49.3 & 45.6 & 44.1 & 60.0 & 59.5 \\
\hline 31 & G & 33.5 & 32.3 & 44.2 & 47.8 & 46.2 & 40.9 & 56.3 & 60.7 \\
\hline 32 & $\mathrm{~K}$ & 23.4 & 27.1 & 33.6 & 34.2 & 28.9 & 19.7 & 47.7 & 49.3 \\
\hline 33 & $\mathrm{~T}$ & 7.1 & 11.5 & 5.5 & 5.9 & 3.7 & 3.0 & 5.0 & 9.2 \\
\hline 34 & $\mathrm{~K}$ & 27.7 & 31.0 & 34.3 & 39.5 & 38.5 & 35.7 & 56.5 & 54.6 \\
\hline 35 & $\mathrm{E}$ & 32.6 & 36.5 & 43.9 & 46.6 & 45.1 & 44.5 & 63.5 & 62.7 \\
\hline 37 & V & 43.7 & 47.7 & 50.0 & 49.2 & 54.0 & 51.1 & 61.5 & 67.7 \\
\hline 38 & $\mathrm{~L}$ & 47.7 & 54.4 & 67.7 & 58.3 & 56.7 & 67.2 & 68.9 & 66.5 \\
\hline 40 & V & 63.1 & 58.2 & 64.2 & 74.4 & 66.7 & 62.6 & 80.5 & 83.2 \\
\hline 41 & G & 47.3 & 47.2 & 54.6 & 58.3 & 46.3 & 47.8 & 61.0 & 56.5 \\
\hline 42 & $S$ & 30.8 & 38.4 & 51.2 & 47.6 & 47.6 & 48.5 & 58.3 & 61.5 \\
\hline 43 & $K$ & 33.0 & 33.5 & 50.3 & 53.7 & 48.2 & 52.1 & 63.5 & 62.5 \\
\hline 44 & $T$ & 31.6 & 33.6 & 48.8 & 55.5 & 54.8 & 57.9 & 67.6 & 61.7 \\
\hline 48 & V & 44.7 & 40.9 & 50.4 & 56.2 & 45.6 & 64.4 & 61.8 & 60.1 \\
\hline 49 & V & 80.4 & 86.3 & 75.8 & 80.9 & 78.6 & 72.8 & 83.6 & 81.6 \\
\hline 50 & $\mathrm{H}$ & 73.5 & 70.6 & 66.5 & 77.0 & 64.8 & 75.4 & 76.6 & 75.6 \\
\hline 51 & $\mathrm{G}$ & 65.5 & 74.1 & 69.4 & 73.8 & 63.6 & 65.1 & 75.4 & 69.5 \\
\hline 52 & V & 92.6 & 94.6 & 84.0 & 92.0 & 90.7 & 86.5 & 90.0 & 89.0 \\
\hline 53 & A & 84.5 & 97.4 & 88.6 & 79.9 & 86.6 & 77.9 & 81.3 & 84.8 \\
\hline
\end{tabular}




\begin{tabular}{|c|c|c|c|c|c|c|c|c|c|}
\hline 54 & $\mathrm{~T}$ & 96.2 & 96.1 & 84.0 & 86.0 & 87.9 & 76.9 & 83.2 & 87.5 \\
\hline 56 & $A$ & 96.7 & 89.0 & 83.6 & 81.9 & 74.5 & 76.8 & 80.8 & 83.9 \\
\hline 57 & $E$ & 72.2 & 65.3 & 75.9 & 59.6 & 55.6 & 44.0 & 51.5 & 56.4 \\
\hline 58 & $\mathrm{~K}$ & 87.8 & 85.3 & 77.5 & 79.8 & 70.2 & 71.6 & 79.4 & 81.7 \\
\hline 59 & $T$ & 71.2 & 74.7 & 75.0 & 66.7 & 70.4 & 66.4 & 80.5 & 65.7 \\
\hline 60 & $\mathrm{~K}$ & 34.5 & 31.9 & 24.6 & 22.7 & 16.5 & 13.9 & 14.9 & 11.5 \\
\hline 62 & $Q$ & 84.6 & 81.4 & 72.8 & 78.0 & 71.7 & 57.5 & 64.4 & 71.7 \\
\hline 64 & $\mathrm{~T}$ & 91.1 & 90.7 & 83.6 & 84.7 & 84.9 & 88.7 & 89.8 & 88.9 \\
\hline 65 & $N$ & 100.8 & 96.7 & 77.9 & 87.7 & 85.2 & 99.3 & 81.8 & 87.6 \\
\hline 66 & $\mathrm{~V}$ & 99.4 & 96.5 & 104.9 & 104.8 & 94.4 & 94.3 & 102.6 & 99.4 \\
\hline 67 & $\mathrm{G}$ & 99.9 & 101.5 & 98.4 & 78.5 & 93.2 & 82.9 & 88.5 & 97.4 \\
\hline 68 & $\mathrm{G}$ & 72.1 & 81.4 & 71.6 & 74.5 & 76.0 & 76.4 & 73.8 & 79.9 \\
\hline 69 & $A$ & 74.0 & 74.7 & 76.4 & 66.1 & 41.6 & 77.8 & 80.6 & 80.7 \\
\hline 70 & $\mathrm{~V}$ & 99.6 & 100.3 & 95.7 & 100.6 & 97.6 & 96.9 & 96.5 & 101.7 \\
\hline 71 & $\mathrm{~V}$ & 100.2 & 93.6 & 92.3 & 100.4 & 105.6 & 111.4 & 106.9 & 102.1 \\
\hline 72 & $\mathrm{~T}$ & 93.9 & 92.6 & 98.0 & 93.7 & 92.6 & 91.3 & 91.0 & 94.0 \\
\hline 73 & $\mathrm{G}$ & 73.2 & 72.3 & 63.6 & 55.7 & 56.8 & 64.9 & 40.6 & 46.9 \\
\hline 74 & $\mathrm{~V}$ & 93.2 & 100.8 & 90.5 & 85.2 & 91.0 & 93.8 & 72.7 & 80.0 \\
\hline 75 & $\mathrm{~T}$ & 92.7 & 97.9 & 99.7 & 92.1 & 100.9 & 98.2 & 98.3 & 97.8 \\
\hline 76 & $A$ & 96.6 & 99.0 & 95.6 & 97.3 & 101.5 & 87.4 & 95.2 & 99.1 \\
\hline 77 & $\mathrm{~V}$ & 99.5 & 98.4 & 95.9 & 96.9 & 91.8 & 85.5 & 93.6 & 96.0 \\
\hline 79 & $Q$ & 90.0 & 88.4 & 93.2 & 90.3 & 86.2 & 83.3 & 95.6 & 91.8 \\
\hline 80 & $\mathrm{~K}$ & 94.5 & 92.5 & 99.0 & 82.3 & 77.4 & 86.1 & 81.9 & 85.4 \\
\hline 81 & $\mathrm{~T}$ & 85.1 & 80.3 & 82.3 & 85.1 & 81.1 & 71.1 & 90.1 & 78.5 \\
\hline 82 & $\mathrm{~V}$ & 78.7 & 82.2 & 79.6 & 74.3 & 70.2 & 73.3 & 69.8 & 77.5 \\
\hline 83 & $\mathrm{E}$ & 47.6 & 43.7 & 51.6 & 69.8 & 55.3 & 60.3 & 74.0 & 61.9 \\
\hline 84 & $\mathrm{G}$ & 34.2 & 39.0 & 44.6 & 55.3 & 53.1 & 44.6 & 74.8 & 67.7 \\
\hline 85 & $A$ & 34.3 & 32.5 & 37.7 & 46.5 & 45.2 & 36.8 & 59.1 & 58.5 \\
\hline 86 & $\mathrm{G}$ & 35.5 & 36.7 & 53.1 & 54.8 & 55.7 & 51.8 & 71.9 & 63.2 \\
\hline 87 & $S$ & 38.0 & 35.9 & 39.6 & 55.3 & 50.9 & 56.0 & 70.5 & 66.0 \\
\hline 88 & 1 & 36.8 & 43.1 & 52.4 & 68.0 & 57.0 & 54.0 & 69.3 & 70.1 \\
\hline 90 & $A$ & 35.2 & 34.0 & 40.5 & 51.4 & 34.1 & 51.2 & 60.3 & 55.9 \\
\hline 92 & $\mathrm{~T}$ & 24.4 & 24.5 & 26.2 & 38.6 & 28.5 & 46.5 & 34.2 & 37.5 \\
\hline 93 & $\mathrm{G}$ & 23.4 & 24.1 & 29.3 & 37.4 & 23.2 & 30.6 & 30.1 & 35.4 \\
\hline 94 & $\mathrm{~F}$ & 22.8 & 19.0 & 25.6 & 37.9 & 26.9 & 26.3 & 27.0 & 31.8 \\
\hline 95 & $\mathrm{~V}$ & 22.5 & 18.1 & 29.6 & 35.5 & 23.0 & 37.2 & 28.0 & 32.1 \\
\hline 96 & $\mathrm{~K}$ & 17.1 & 21.9 & 29.4 & 31.2 & 23.2 & 31.2 & 25.5 & 28.7 \\
\hline 97 & $\mathrm{~K}$ & 10.5 & 13.6 & 18.8 & 18.4 & 17.4 & 15.1 & 13.1 & 14.7 \\
\hline 98 & $D$ & 1.5 & 7.8 & 8.8 & 7.7 & 5.2 & 0.1 & 0.5 & 2.8 \\
\hline 99 & $Q$ & 10.4 & 7.0 & 7.6 & 5.9 & 2.7 & 3.1 & 3.2 & 7.4 \\
\hline 100 & $\mathrm{~L}$ & 5.0 & 4.2 & 5.9 & 2.7 & 6.2 & 3.6 & 1.9 & 6.4 \\
\hline 101 & $\mathrm{G}$ & 0.2 & 5.9 & 8.7 & 6.4 & 1.4 & 4.5 & 5.7 & 1 \\
\hline 107 & $A$ & 24.3 & 21.6 & 20.6 & 21.2 & 22.0 & 36.0 & 4.4 & U \\
\hline
\end{tabular}




\begin{tabular}{rlrrrrrrrr}
109 & Q & 9.6 & 8.5 & 18.3 & 9.2 & 5.1 & 1.4 & 8.4 & 9.1 \\
110 & $\mathrm{E}$ & 7.7 & 13.4 & 14.2 & 17.2 & 9.1 & 13.7 & 9.5 & 12.0 \\
111 & $\mathrm{G}$ & 8.9 & 5.3 & 9.7 & 15.8 & 7.9 & 0.4 & 9.4 & 9.3 \\
112 & $\mathrm{I}$ & 8.7 & 7.1 & 9.1 & 5.7 & 6.2 & 0.8 & 5.3 & 7.7 \\
113 & $\mathrm{~L}$ & 3.0 & 2.3 & 1.1 & 2.6 & -2.0 & -0.3 & 3.7 & 2.3 \\
115 & $\mathrm{D}$ & 8.2 & 8.3 & 7.6 & 13.1 & 6.9 & -6.1 & 4.1 & 6.8 \\
116 & $\mathrm{M}$ & -3.1 & 0.7 & -1.9 & -1.1 & -0.5 & 0.6 & 0.6 & -1.4 \\
118 & $\mathrm{~V}$ & 9.1 & 7.4 & 0.0 & 7.1 & 4.9 & 6.8 & 6.4 & 5.3 \\
119 & $\mathrm{D}$ & 12.5 & 10.8 & 16.0 & 18.2 & 7.4 & 13.3 & 12.0 & 12.2 \\
121 & $\mathrm{D}$ & 5.9 & 13.5 & 10.9 & 9.9 & 9.7 & 3.5 & 5.8 & 16.3 \\
122 & $\mathrm{~N}$ & 2.4 & 4.4 & 7.6 & 5.7 & 0.1 & -4.5 & 4.3 & 1.5 \\
127 & $\mathrm{M}$ & 0.0 & 2.7 & -0.5 & 0.7 & -0.6 & -0.5 & 0.8 & -2.3 \\
129 & $\mathrm{~S}$ & 2.1 & 6.9 & 7.8 & 3.2 & 7.6 & 14.7 & 5.0 & 5.4 \\
130 & $\mathrm{E}$ & 6.1 & 4.8 & 1.5 & 6.2 & 3.9 & 1.9 & 5.3 & 4.8 \\
131 & $\mathrm{E}$ & 6.6 & 3.6 & 5.5 & 7.0 & 5.6 & 4.2 & 8.5 & 11.7 \\
132 & $\mathrm{G}$ & 10.3 & 10.6 & 9.4 & 10.6 & 7.2 & 7.7 & 7.5 & 5.3 \\
133 & $\mathrm{Y}$ & 7.5 & 7.7 & 3.9 & 8.8 & 6.1 & 7.4 & 10.0 & 6.3 \\
134 & $\mathrm{E}$ & 7.1 & 7.6 & 0.7 & -0.1 & 5.8 & 9.1 & -1.1 & 3.7 \\
136 & $\mathrm{Y}$ & 4.3 & 4.9 & 6.0 & 2.0 & 4.1 & 6.8 & 2.5 & 7.0 \\
137 & $\mathrm{E}$ & 5.0 & 0.6 & 3.9 & 5.1 & 3.4 & 5.4 & 2.5 & 6.1 \\
139 & $\mathrm{E}$ & 11.8 & 11.7 & 9.7 & 8.5 & 5.4 & 3.8 & 10.1 & 9.2 \\
140 & $\mathrm{~A}$ & 8.0 & 7.4 & 10.4 & 6.2 & 4.9 & 8.3 & 10.2 & 5.3 \\
\hline
\end{tabular}

Table 7. Back-fitted initial protonation levels of brain-extract seeded aSyn fibrils.

Values report the residue-specific protonation levels $\left[\%{ }^{1} \mathrm{H}\right]$ of brain-extract seeded aSyn fibrils amplified from distinct patient brains (Table 1) after 1.5 days of forward-exchange in $99.9 \%$ $\mathrm{D}_{2} \mathrm{O}$ supplemented with $0.1 \%$ formic acid $(\mathrm{pD} 4.0)$ at $37^{\circ} \mathrm{C}$. 
7.6.2 In vitro aSyn polymorph fibrils

\begin{tabular}{|c|c|c|c|c|c|c|c|}
\hline ID & AA & $\begin{array}{l}\text { de novo } \\
\text { hsAsyn }\end{array}$ & hsAsyn I & hsAsyn II & $\begin{array}{l}\text { high salt } \\
\text { [HEPES] }\end{array}$ & IsAsyn I & IsAsyn II \\
\hline 1 & $M$ & 31.5 & 30.8 & 31.5 & 22.3 & 43.6 & 43.4 \\
\hline 3 & V & 22.2 & 26.2 & 24.5 & 37.8 & 85.2 & 77.9 \\
\hline 4 & $\mathrm{~F}$ & 17.9 & 21.1 & 21.3 & 34.3 & 89.4 & 85.5 \\
\hline 5 & $M$ & 19.0 & 20.1 & 20.8 & 39.2 & 91.0 & 85.7 \\
\hline 6 & $\mathrm{~K}$ & 28.1 & 28.5 & 25.9 & 47.7 & 85.2 & 88.2 \\
\hline 7 & G & 18.0 & 21.7 & 20.2 & 27.4 & 72.4 & 68.2 \\
\hline 9 & $S$ & 19.7 & 20.0 & 19.0 & 36.5 & 86.7 & 76.4 \\
\hline 11 & $A$ & 13.6 & 6.9 & 3.5 & 27.5 & 94.9 & 91.9 \\
\hline 12 & $\mathrm{~K}$ & 4.5 & 14.9 & 13.0 & 25.4 & 84.5 & 75.7 \\
\hline 15 & V & 22.6 & 26.5 & 21.3 & 37.0 & 79.1 & 75.8 \\
\hline 16 & V & 22.3 & 24.9 & 21.1 & 38.2 & 97.8 & 88.4 \\
\hline 17 & $A$ & 27.2 & 25.5 & 23.9 & 40.4 & 94.5 & 94.6 \\
\hline 18 & $A$ & 31.9 & 31.3 & 27.6 & 42.1 & 87.0 & 86.8 \\
\hline 20 & $E$ & 19.3 & 6.2 & 5.0 & 34.4 & 91.3 & 90.6 \\
\hline 21 & $\mathrm{~K}$ & 18.4 & 15.4 & 13.5 & 36.3 & 93.4 & 85.5 \\
\hline 22 & $T$ & 30.6 & 27.1 & 27.0 & 35.0 & 78.8 & 75.8 \\
\hline 26 & V & 16.1 & 28.9 & 26.0 & 36.7 & 79.0 & 82.8 \\
\hline 27 & $A$ & 17.9 & 28.8 & 26.7 & 35.5 & 75.9 & 71.6 \\
\hline 29 & $A$ & 15.4 & 27.9 & 25.8 & 34.0 & 52.8 & 54.4 \\
\hline 31 & G & 18.7 & 30.3 & 26.0 & 33.9 & 18.9 & 18.7 \\
\hline 32 & $\mathrm{~K}$ & 18.7 & 26.4 & 25.2 & 31.0 & 17.6 & 19.6 \\
\hline 33 & $T$ & 8.4 & 23.4 & 20.1 & 22.6 & 25.9 & 21.6 \\
\hline 34 & $\mathrm{~K}$ & 12.6 & 8.5 & 8.7 & 30.6 & 17.5 & 19.6 \\
\hline 37 & V & 42.2 & 46.2 & 41.2 & 35.0 & 32.6 & 34.6 \\
\hline 38 & $L$ & 84.9 & 70.2 & 70.8 & 67.2 & 89.9 & 85.6 \\
\hline 40 & V & 88.9 & 78.8 & 74.8 & 74.4 & 85.8 & 88.1 \\
\hline 41 & G & 91.3 & 77.2 & 66.5 & 48.4 & 85.3 & 95.7 \\
\hline 42 & $S$ & 69.7 & 65.9 & 69.6 & 55.3 & 88.6 & 87.6 \\
\hline 43 & $K$ & 79.6 & 85.3 & 85.5 & 70.6 & 85.4 & 91.1 \\
\hline 44 & $T$ & 68.0 & 75.2 & 77.6 & 76.8 & 100.4 & 98.9 \\
\hline 49 & $\mathrm{~V}$ & 94.3 & 97.3 & 92.5 & 93.3 & 94.6 & 91.4 \\
\hline 50 & $\mathrm{H}$ & 94.0 & 86.8 & 89.9 & 90.9 & 87.6 & 90.2 \\
\hline 51 & G & 87.9 & 92.0 & 89.7 & 84.9 & 88.7 & 91.9 \\
\hline 52 & V & 99.1 & 101.4 & 101.4 & 99.9 & 99.4 & 104.7 \\
\hline 53 & $A$ & 91.8 & 93.9 & 87.8 & 95.3 & 91.8 & 89.4 \\
\hline 54 & $\mathrm{~T}$ & 96.6 & 94.9 & 97.5 & 94.4 & 92.3 & 95.5 \\
\hline 56 & $A$ & 96.1 & 93.8 & 93.2 & 94.6 & 89.8 & 98.1 \\
\hline 57 & $E$ & 84.7 & 86.6 & 90.3 & 85.3 & 73.9 & 81.8 \\
\hline 58 & $\mathrm{~K}$ & 91.3 & 89.0 & 87.5 & 80.5 & 87.8 & 86.3 \\
\hline
\end{tabular}




\begin{tabular}{|c|c|c|c|c|c|c|c|}
\hline 59 & $\mathrm{~T}$ & 62.4 & 37.1 & 36.0 & 46.2 & 77.2 & 78.1 \\
\hline 60 & $K$ & 72.6 & 80.7 & 88.7 & 58.7 & 34.2 & 36.7 \\
\hline 62 & $Q$ & 84.9 & 84.2 & 89.2 & 85.3 & 81.8 & 86.3 \\
\hline 64 & $\mathrm{~T}$ & 94.0 & 93.8 & 96.9 & 92.3 & 86.8 & 89.8 \\
\hline 65 & $\mathrm{~N}$ & 95.4 & 91.3 & 87.6 & 88.3 & 88.4 & 93.3 \\
\hline 66 & $\mathrm{~V}$ & 99.9 & 99.5 & 94.7 & 88.1 & 92.7 & 92.1 \\
\hline 67 & $\mathrm{G}$ & 91.4 & 90.0 & 89.6 & 94.3 & 64.1 & 69.8 \\
\hline 68 & $\mathrm{G}$ & 83.8 & 83.4 & 80.9 & 64.6 & 33.1 & 28.2 \\
\hline 69 & $A$ & 76.1 & 79.5 & 85.4 & 74.9 & 38.7 & 37.4 \\
\hline 70 & $\mathrm{~V}$ & 100.8 & 96.9 & 99.9 & 92.0 & 91.4 & 97.2 \\
\hline 71 & $\mathrm{~V}$ & 98.5 & 94.5 & 88.8 & 104.5 & 93.9 & 90.5 \\
\hline 72 & $\mathrm{~T}$ & 98.5 & 96.6 & 94.9 & 98.4 & 91.4 & 84.4 \\
\hline 73 & $\mathrm{G}$ & 68.7 & 48.3 & 48.6 & 82.4 & 87.9 & 81.5 \\
\hline 74 & $\mathrm{~V}$ & 99.6 & 104.0 & 100.4 & 97.7 & 98.3 & 97.4 \\
\hline 75 & $\mathrm{~T}$ & 92.1 & 95.9 & 95.0 & 95.9 & 94.4 & 96.5 \\
\hline 76 & $A$ & 99.4 & 96.6 & 97.4 & 98.2 & 98.1 & 100.9 \\
\hline 77 & V & 88.6 & 88.2 & 85.3 & 85.2 & 94.8 & 87.8 \\
\hline 79 & $Q$ & 94.7 & 94.1 & 95.0 & 91.8 & 103.7 & 85.8 \\
\hline 80 & $\mathrm{~K}$ & 97.5 & 92.9 & 97.2 & 90.5 & 94.2 & 92.3 \\
\hline 81 & $\mathrm{~T}$ & 100.3 & 97.8 & 100.8 & 98.9 & 89.6 & 96.2 \\
\hline 82 & $\mathrm{~V}$ & 87.6 & 91.0 & 87.1 & 82.8 & 84.1 & 86.2 \\
\hline 83 & $E$ & 94.0 & 92.2 & 90.9 & 70.9 & 88.7 & 85.4 \\
\hline 84 & $\mathrm{G}$ & 53.5 & 66.7 & 62.6 & 41.8 & 69.1 & 65.7 \\
\hline 85 & $A$ & 20.2 & 7.3 & 5.7 & 9.2 & 64.2 & 60.9 \\
\hline 86 & $\mathrm{G}$ & 85.3 & 92.2 & 90.8 & 76.4 & 91.1 & 91.8 \\
\hline 87 & $S$ & 88.9 & 92.6 & 91.9 & 89.8 & 82.5 & 85.3 \\
\hline 88 & 1 & 84.2 & 83.4 & 81.9 & 78.4 & 89.1 & 89.8 \\
\hline 90 & $A$ & 78.1 & 78.4 & 77.7 & 75.4 & 81.4 & 86.2 \\
\hline 92 & $\mathrm{~T}$ & 88.0 & 82.2 & 80.9 & 95.7 & 86.5 & 91.5 \\
\hline 93 & $\mathrm{G}$ & 69.5 & 54.6 & 55.9 & 87.1 & 88.5 & 86.6 \\
\hline 94 & $\mathrm{~F}$ & 69.8 & 50.2 & 51.7 & 76.9 & 85.3 & 83.0 \\
\hline 95 & $\mathrm{~V}$ & 65.6 & 45.8 & 48.7 & 75.9 & 89.2 & 90.6 \\
\hline 96 & $\mathrm{~K}$ & 61.6 & 37.2 & 37.1 & 67.4 & 87.1 & 86.5 \\
\hline 97 & $\mathrm{~K}$ & 38.7 & 16.5 & 15.7 & 34.1 & 37.9 & 37.2 \\
\hline 98 & $\mathrm{D}$ & 5.6 & 6.5 & 4.0 & 8.0 & 0.6 & 5.7 \\
\hline 99 & $Q$ & 14.1 & 9.4 & 6.8 & 7.8 & 15.0 & 17.5 \\
\hline 100 & $\mathrm{~L}$ & 9.7 & 8.4 & 7.5 & 10.1 & 12.2 & 16.0 \\
\hline 101 & $\mathrm{G}$ & 8.6 & 6.5 & 4.1 & 7.3 & 10.0 & 9.5 \\
\hline 107 & $A$ & 18.9 & 14.5 & 13.9 & 13.4 & 23.0 & 13.7 \\
\hline 109 & $Q$ & 7.2 & 9.2 & 7.6 & 10.1 & 13.3 & 13.7 \\
\hline 110 & $\mathrm{E}$ & 22.9 & 22.6 & 15.6 & 10.6 & 19.4 & 20.9 \\
\hline 111 & $\mathrm{G}$ & 11.9 & 12.0 & 11.0 & 9.9 & 12.7 & 10.5 \\
\hline 112 & 1 & 5.2 & 7.8 & 5.3 & 9.9 & 19.4 & 16.5 \\
\hline
\end{tabular}




\begin{tabular}{rlrrrrrr}
113 & $\mathrm{~L}$ & 3.2 & 3.0 & 3.2 & 0.9 & 10.8 & 11.0 \\
115 & $\mathrm{D}$ & 11.9 & 11.3 & 12.4 & 14.4 & 13.4 & 12.3 \\
116 & $\mathrm{M}$ & -2.3 & -1.8 & -1.3 & 0.1 & -3.5 & -3.0 \\
118 & $\mathrm{~V}$ & 5.2 & 5.2 & 5.6 & 11.7 & 9.9 & 5.7 \\
119 & $\mathrm{D}$ & 14.5 & 11.7 & 8.6 & 11.0 & 13.3 & 14.7 \\
121 & $\mathrm{D}$ & 8.4 & 8.7 & 6.1 & 10.3 & 15.8 & 8.5 \\
122 & $\mathrm{~N}$ & 2.0 & 1.1 & 1.5 & 3.1 & -2.0 & -1.5 \\
127 & $\mathrm{M}$ & -0.8 & 0.1 & 0.5 & -1.2 & 0.6 & -4.0 \\
129 & $\mathrm{~S}$ & 7.1 & 6.4 & 4.3 & 7.5 & 5.8 & 5.7 \\
130 & $\mathrm{E}$ & 5.4 & 7.8 & 5.1 & 9.9 & 8.4 & 7.9 \\
131 & $\mathrm{E}$ & 9.0 & 8.1 & 7.4 & 9.3 & 9.6 & 8.2 \\
133 & $\mathrm{Y}$ & 5.7 & 7.3 & 4.5 & 8.1 & 8.9 & 6.0 \\
134 & $\mathrm{E}$ & 6.2 & 7.1 & 6.6 & 7.2 & 4.1 & 7.0 \\
136 & $\mathrm{Y}$ & 9.6 & 5.9 & 6.1 & 6.4 & 6.3 & 6.5 \\
137 & $\mathrm{E}$ & 4.3 & 6.1 & 5.4 & 5.0 & 7.4 & 9.2 \\
139 & $\mathrm{E}$ & 9.1 & 8.8 & 7.2 & 9.5 & 6.9 & 7.6 \\
140 & $\mathrm{~A}$ & 8.0 & 6.6 & 7.9 & 7.2 & 6.9 & 7.6 \\
\hline
\end{tabular}

Table 8. Back-fitted initial protonation levels of aSyn in vitro polymorph fibrils.

Values report the residue-specific protonation levels $\left[\%{ }^{1} \mathrm{H}\right]$ of in vitro aSyn polymorph fibrils after 1.5 days of forward-exchange in $99.9 \% \mathrm{D}_{2} \mathrm{O}$ supplemented with $0.1 \%$ formic acid ( $p D 4.0$ ) at $37^{\circ} \mathrm{C}$. 


\subsection{Dipolar modulation of aSyn in vitro polymorphs analyzed by DEERNet neural network}

Here, an alternative processing of PELDOR experiments to the standard Tikhonov regularization with L-curve selection (Figure 3-25 a) is shown for the two in vitro aSyn polymorphs, hsAsyn and IsAsyn (Figure 7-3). The approach is based on the DEERNet neural network included in DEERAnalysis2018 and described in detail by Worswick et al. ${ }^{138}$.


Figure 7-3. Dipolar modulation of aSyn fibrils using DEERNet.

Dipolar modulation (corrected for background; fit in blue/red, same as in Figure 3-25 a) and normalized distance distribution determined by $34 \mathrm{GHz}$ four-pulse PELDOR experiments for hsAsyn (top, blue) and IsAsyn (bottom, red) analyzed by deep neural network processing ${ }^{138}$ using DEERAnalysis2018 and DEERNet with "broad peak" model.

The distance distribution obtained by Tikhonov regularization with L-curve selection is faithfully reproduced on the same raw dataset with highly efficient suppression of distance artifacts. 


\subsection{Biophysical characterization of $\alpha$-synuclein membrane} interactions and the close homologue $\beta$-synuclein

A biophysical characterization of $\mathrm{N}$-terminally acetylated $\alpha$-synuclein and its close homologue $\beta$-synuclein ${ }^{201}$ in the presence of mildly negatively charged small unilamellar vesicles (SUVs) of DOPE/DOPS/DOPC ${ }^{202}(5: 3: 12$, w/w) by twodimensional ${ }^{1} \mathrm{H}^{-15} \mathrm{~N}$ NMR correlation spectroscopy and circular dichroism (CD) was conducted. $\mathrm{N}$-terminally acetylated $\beta$-synuclein (bSyn) was expressed and purified as described by Miotto et al. ${ }^{203}$. The SUVs were generated following a published procedure $^{202}$ using a 1:1 mixture of DOPC and coagulation reagent I containing DOPE/DOPS/DOPC (5:3:12, w/w), both purchased from Avanti Polar Lipids. The total phospholipid concentration of the SUV stock was $85 \mathrm{mM}(6.6 \% \mathrm{w} / \mathrm{v})$.

\subsubsection{Protein-membrane interactions by NMR spectroscopy}

The ${ }^{1} \mathrm{H}-{ }^{15} \mathrm{~N}$ HSQC spectra of the SUV titration experiment (Figure 7-4) were acquired on a $600 \mathrm{MHz}$ Bruker NMR spectrometer equipped with a cryoprobe. MES was used as a buffer system (20 mM MES, $100 \mathrm{mM} \mathrm{NaCl}, \mathrm{pH} 6.5,0.02 \% \mathrm{NaN}_{3}$ ). The assignment for $\alpha$ - and $\beta$-synuclein was kindly provided by Dr. Fernández.

a

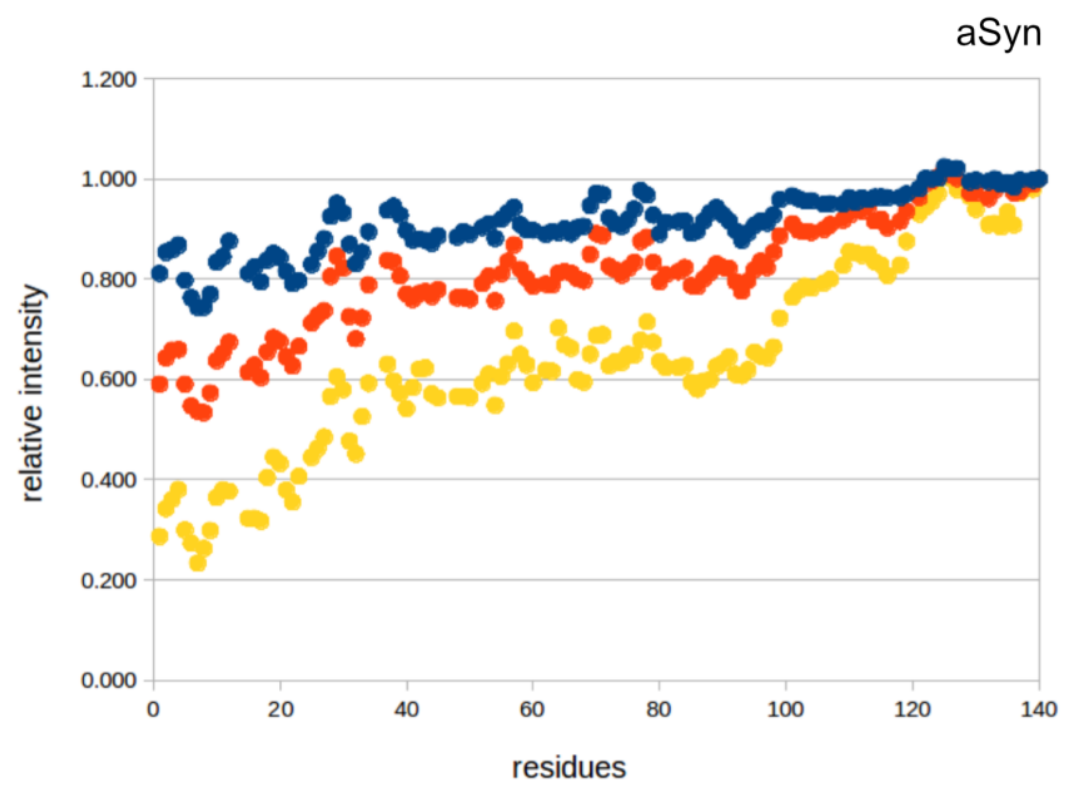


b



Figure 7-4. SUV titration of $\alpha$ - and $\beta$-synuclein by NMR spectroscopy.

(a) $100 \mu \mathrm{M}$ aSyn and (b) $100 \mu \mathrm{M}$ bSyn in MES buffer (20 mM MES, $100 \mathrm{mM} \mathrm{NaCl}, \mathrm{pH}$ 6.5, 0.02 $\% \mathrm{NaN}_{3}$ ) was titrated at $25^{\circ} \mathrm{C}$ with 25 -fold (blue), 50 -fold (red) and 100 -fold (yellow) excess of SUVs, DOPE/DOPS/DOPC (5:3:12, w/w). The ${ }^{1} \mathrm{H}-{ }^{15} \mathrm{~N}$ HSQC spectra were acquired at $600 \mathrm{MHz}$ and the intensity values of free aSyn (a) as well as free bSyn (b) residues ( $\mathrm{l}_{0}$ ) were used to determine the relative intensity ratio $(1 / 10)$. The relative intensity values were averaged over the residues $\mathrm{i}-1$, $\mathrm{i}$ and $\mathrm{i}+1$. The non-perturbed C-terminal A140 for aSyn and A134 for bSyn were set to 1 .

\subsubsection{Protein-membrane interactions by CD spectroscopy}

To further characterize the binding of $\alpha$ - and $\beta$-synuclein to the phospholipid bilayer of SUVs, mimicking biological membranes, CD spectroscopy was chosen. The membrane bound population of $\alpha$ - and $\beta$-synuclein forms an $\alpha$-helical conformation ${ }^{32,33}$, while unbound populations remain intrinsically disordered. Monitoring the buildup of mean residue ellipticity $\left(\theta_{\mathrm{MR}}\right)$ at $222 \mathrm{~nm}$, due to the formation of $\alpha$-helical membrane bound conformations (Figure 7-5 a), an apparent dissociation constant $\left(\mathrm{K}_{\mathrm{D}, \mathrm{app}}\right)$ can be derived (Figure 7-5 b) established and shown for aSyn by Pfefferkorn et al. ${ }^{204}$. For a quantitative analysis, the spectra do not meet the required quality. The $\alpha$ - and $\beta$-synuclein CD spectra (Figure 7-5 a) do not show a single isodichroic point that would indicate a two-state conformational change from intrinsically disordered to $\alpha$-helical. This might be due to weak protein-lipid interactions, the DOPE/DOPS/DOPC (5:3:12, w/w) SUV system is only weakly negatively charged in its DOPS moiety, requiring high excess of SUVs (up to 700 -fold). The derived values 
for $\mathrm{K}_{\mathrm{D}, \mathrm{app}}$ can only be used qualitatively, for aSyn a value of $10.6 \mathrm{mM}$ and for bSyn a value of $9.2 \mathrm{mM}$ was derived.

a
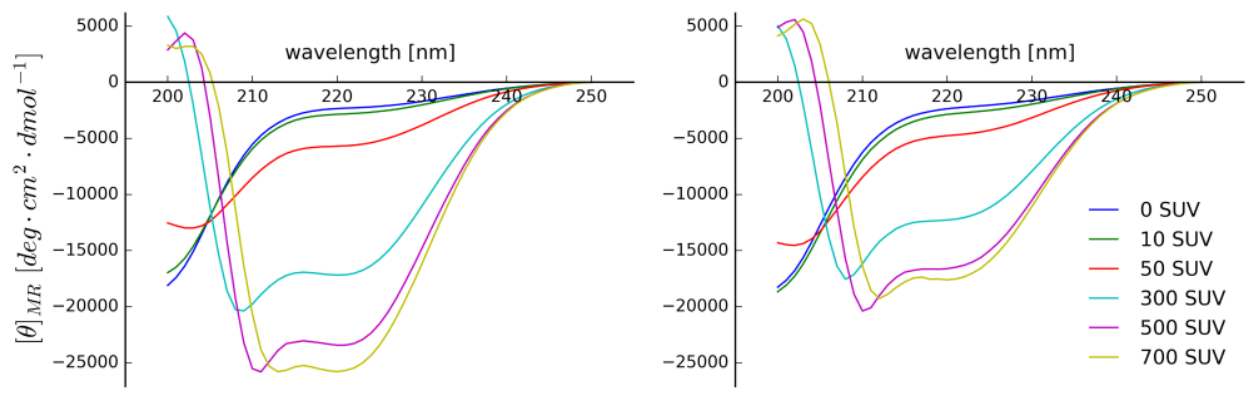

b

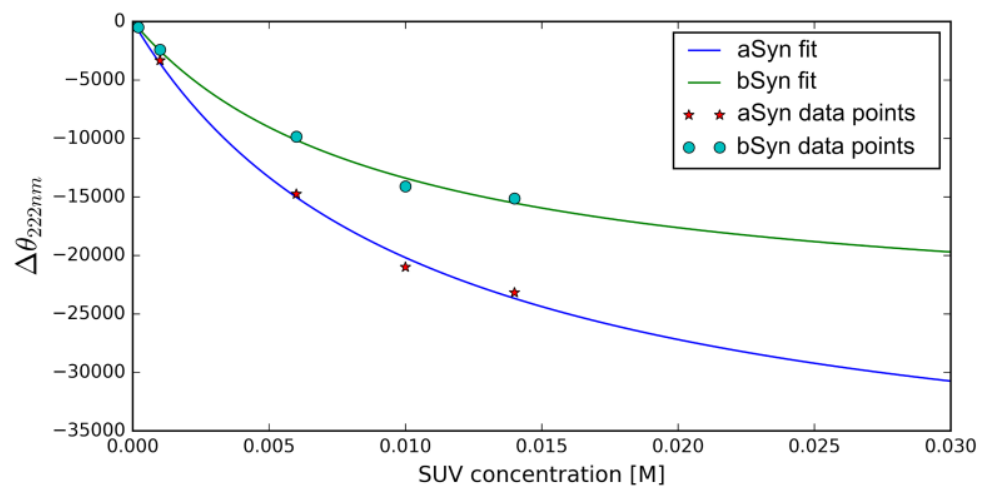

Figure 7-5. SUV titration of $\alpha$ - and $\beta$-synuclein by CD spectroscopy.

(a) $20 \mu \mathrm{M}$ aSyn (left) and $20 \mu \mathrm{M}$ bSyn (right) in low ionic strength NaP buffer (10 mM NaP, pH 6.5) were titrated at $25^{\circ} \mathrm{C}$ with 10- to 700 -fold excess of SUVs, DOPE/DOPS/DOPC (5:3:12, w/w). Reference spectrum in blue, 10- (green), 50- (red), 300- (cyan), 500- (purple) and 700fold (olive). (b) The mean residue ellipticity values are plotted against the absolute SUV concentration in mol/l. The datasets were fitted as described by Pfefferkorn et al. ${ }^{204}$. Analysis and plotting were performed using Jupyter notebook (Python 2.7, SciPy's least-square fitting library "curve_fit"). All experiments were recorded on a JASCO J-1500 CD spectrometer.

The data obtained by NMR spectroscopy suggest that there are differences in the binding mode to this particular system of SUVs between aSyn and bSyn. The intensity decrease is more pronounced for membrane-interacting residues of aSyn than for bSyn. This might suggest a higher population of aSyn bound to SUVs. CD spectroscopy also reports less $\alpha$-helical content for bSyn. Counterintuitively, the interaction of aSyn and bSyn with SUVs occur though with similar affinities. Additional experiments are required to better characterize interactions with SUVs and work out robust differences between $\alpha$ - and $\beta$-synuclein. 